\title{
Evolution of Chain Dynamics and Oxidation States with Increasing Chain Length for a Donor-Acceptor Conjugated Oligomer Series
}

Saadia Chaudhry ${ }^{1}$, Yukun $\mathrm{Wu}^{1}$, Zhiqiang $\mathrm{Cao}^{2}, \mathrm{Shi}^{3}{ }^{3}$, Jodie L. Canada ${ }^{3}$, Xiaodan $\mathrm{Gu}^{2}$, Chad Risko ${ }^{3 *}$ and Jianguo $\mathrm{Mei}^{1 *}$

${ }^{1}$ Department of Chemistry, Purdue University West Lafayette, IN 47907, USA

${ }^{2}$ School of Polymer Science and Engineering, the University of Southern Mississippi, Hattiesburg, MS 39406, USA

${ }^{3}$ Department of Chemistry \& Center for Applied Energy Research (CAER), University of Kentucky, Lexington, KY, 40506, USA

\section{Supporting Information}

Table of Contents

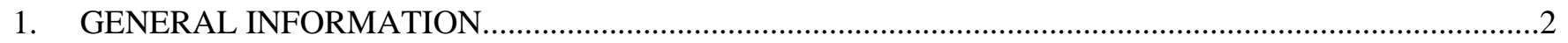

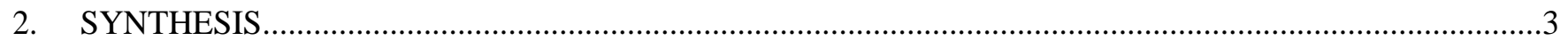

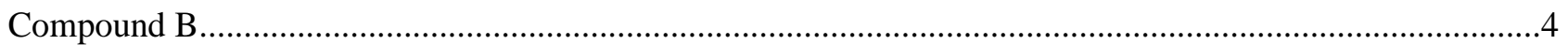

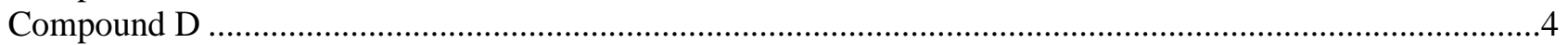

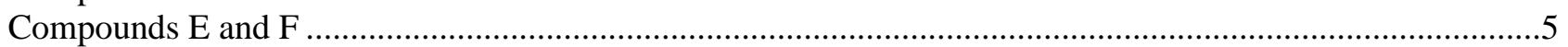

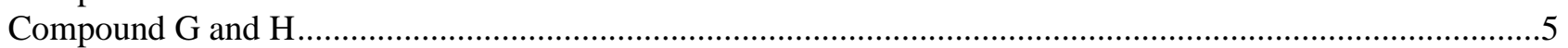

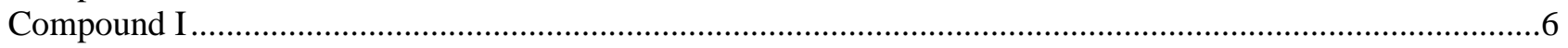

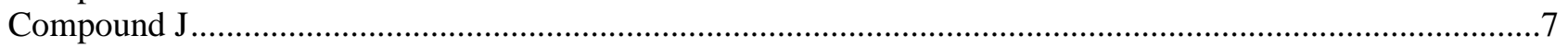

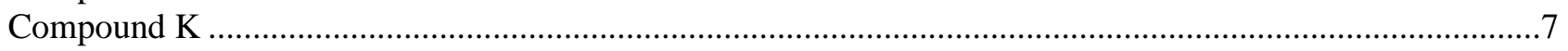

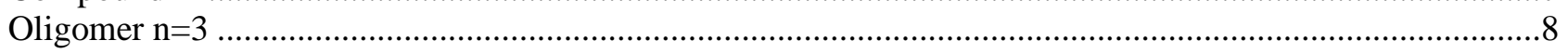

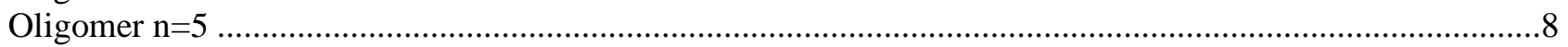

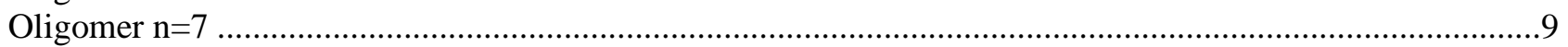

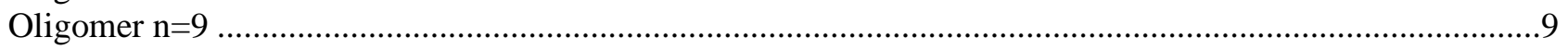

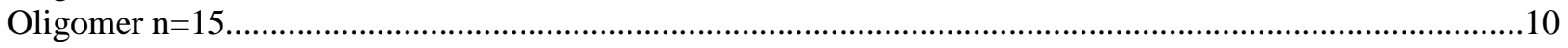

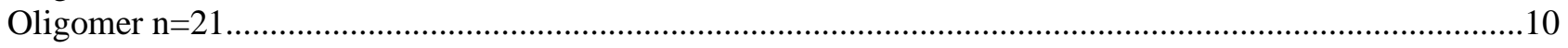

Recycling Preparative HPLC Chromatograms for Polymers .............................................................. 12

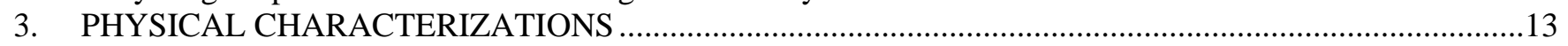

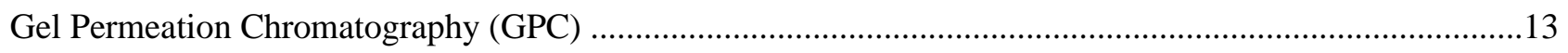

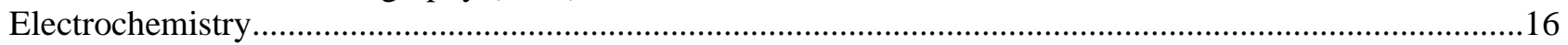

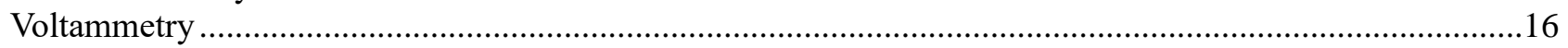

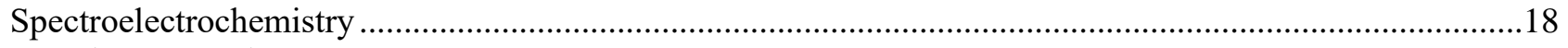

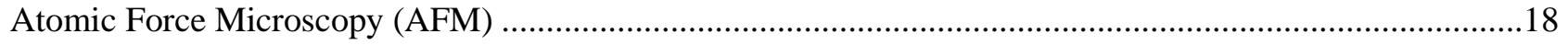

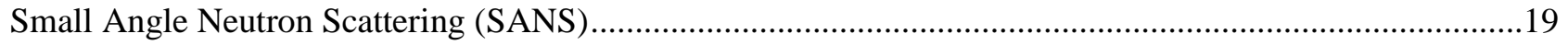

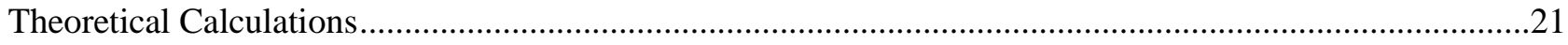

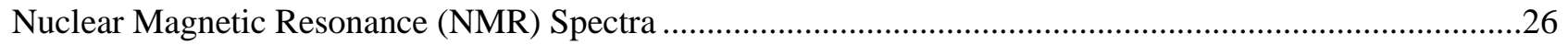




\section{GENERAL INFORMATION}

General Considerations. All syntheses were carried out using standard Schlenk or glovebox techniques under an atmosphere of N2. Solvents were dried and degassed by passing through a column of activated alumina, sparging with N2 gas, and, storing over dry $3 \AA$ molecular sieves for at least $72 \mathrm{~h}$ before use. Deuterated solvent was purchased from Cambridge Isotope Laboratories, Inc. All reagents and starting materials were purchased from commercial vendors and used without further purification unless otherwise noted.

Physical Methods. ${ }^{1} \mathrm{H}$ NMR spectra were collected at room temperature on Bruker $400 \mathrm{MHz}, 500 \mathrm{MHz}$, or 800 $\mathrm{MHz}$ spectrometers. ${ }^{1} \mathrm{H}$ and ${ }^{13} \mathrm{C}$ NMR spectra are reported in parts per million relative to tetramethylsilane, using the residual solvent resonances as an internal standard. High resolution mass data were obtained using an Agilent 6320 LC-MS/MS for HR-APCI and an Applied Biosystems Voyager System 6270 for MALDI. Sample dispersity and polymer molecular weights were obtained using a TOSOH GPC with THF solvent vs. polystyrene standards at $40^{\circ} \mathrm{C}$. UV-vis-NIR measurements were acquired on an Agilent Technologies Cary 6000i spectrophotometer $(350-2850 \mathrm{~nm})$ using a $1-\mathrm{cm}$ two-window quartz cuvette. Electrochemistry was performed using a BioLogic SP-150 and CHI-660.

Abbreviations. 3,4-propylenedioxythiophene (ProDOT), benzothiadiazole (BTD), dichloromethane (DCM), tetrahydrofuran (THF). 


\section{SYNTHESIS}

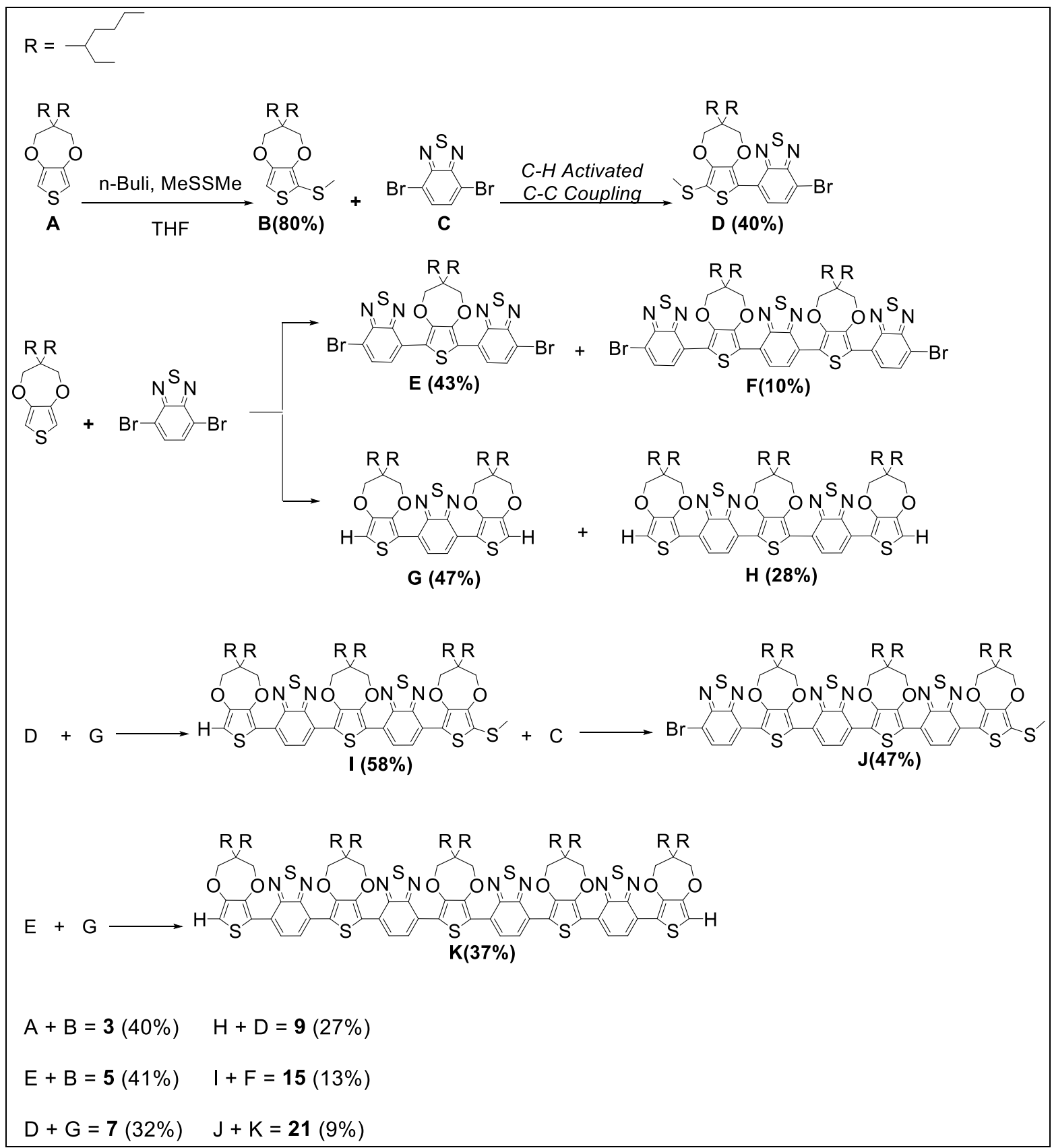

Scheme S1. Synthesis of the $\boldsymbol{n P B}$ oligomers. a) n-BuLi, $\mathrm{CH}_{3} \mathrm{SSCH}_{3}$, b) C-H Activation Conditions: $\mathrm{Pd}(\mathrm{OAc})_{2}$, $(\mathrm{o}-\mathrm{MeOPh})_{3} \mathrm{P}$, pivalic acid, $\mathrm{Cs}_{2} \mathrm{CO}_{3}$. 


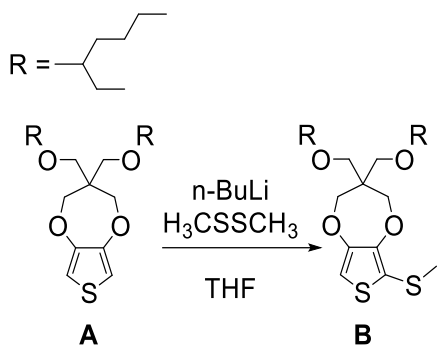

\section{Compound B}

To a THF solution $(75 \mathrm{~mL})$ of $\mathbf{A}(9.90 \mathrm{~g}, 22.5 \mathrm{mmol}, 1.0$ equiv) was added $9.88 \mathrm{ml}$ of n-butyllithium (2.5 M, $24.7 \mathrm{mmol}, 1.1$ equiv) in hexane at $-78^{\circ} \mathrm{C}$ under $\mathrm{N}_{2}$. After stirring for $120 \mathrm{~min}$ at $0^{\circ} \mathrm{C}$, dimethyldisulfide $(2.22 \mathrm{ml}$, $24.7 \mathrm{mmol}, 1.1$ equiv) was added dropwise to the reaction mixture and then allowed to warm to room temperature. After stirring overnight, the reaction mixture was quenched with water, extracted with hexane, and dried over $\mathrm{Na}_{2} \mathrm{SO}_{4}$. The crude product was concentrated under vacuum and passed through a short alumina column using hexanes:ethyl acetate (75:1). After solvent evaporation, the residue was separated by preparative HPLC (polystyrene/divinylbenzene stationary phase) with $\mathrm{CHCl}_{3}$ to give $\mathbf{B}$ as a light-yellow oil ( $8.76 \mathrm{~g}, 80 \%$ yield).

- $\quad{ }^{1} \mathrm{H}$ NMR (300 MHz, $\left.\mathrm{CDCl}_{3}, \mathrm{ppm}\right) \delta: 6.44(\mathrm{~s}, 1 \mathrm{H}), 4.09$ (s, 2H), 4.00 (s, 2H), 3.47 (s, 4H), 3.27 (d, J = 5.7 $\mathrm{Hz}, 4 \mathrm{H}), 2.37(\mathrm{~s}, 3 \mathrm{H}), 1.35-1.23(\mathrm{~m}, 20 \mathrm{H}), 0.90-0.84(\mathrm{~m}, 13 \mathrm{H})$.

- ${ }^{13} \mathrm{C} \mathrm{NMR}\left(101 \mathrm{MHz}, \mathrm{CDCl}_{3}, \mathrm{ppm}\right) \delta 150.63,149.15,106.15,74.18,73.93,73.61,69.73,47.83,39.51,30.57$, 29.03, 23.89, 23.00, 21.12, 14.02, 11.06.

- $\quad H R M S(E S I),[M+H]+m / z$ calcd. for $\mathrm{C}_{26} \mathrm{H}_{47} \mathrm{O}_{4} \mathrm{~S}_{2}: 487.2910$; found 487.2902.

\section{General C-H Activation Conditions}

In a $25 \mathrm{~mL}$ Schlenk tube, $\mathrm{Pd}(\mathrm{OAc})_{2}(4-8 \mathrm{~mol} \%),(\mathrm{o}-\mathrm{MeOPh})_{3} \mathrm{P}(8-16 \mathrm{~mol} \%)$, pivalic acid $(0.5$ per $\mathrm{C}-\mathrm{H}$ monomer equivalence) and $\mathrm{Cs}_{2} \mathrm{CO}_{3}$ (1.5 per $\mathrm{C}-\mathrm{H}$ monomer equivalence) were added, vacuumed for 30 minutes, and then subjected to $\mathrm{N}_{2}$ purge cycles three times. Anhydrous toluene $(0.1 \mathrm{M}-0.5 \mathrm{M})$ was added via needle and syringe. The reaction was heated at $110^{\circ} \mathrm{C}$ in an oil bath, or until the limiting reagent was consumed per TLC analysis. Upon completion, the reaction mixture was quenched with water and extracted with dichloromethane. The organic layer was washed with water twice, then once with brine. The organic layer was separated and dried over $\mathrm{MgSO}_{4}$ or $\mathrm{Na}_{2} \mathrm{SO}_{4}$, then filtered and concentrated under reduced pressure. The crude product was purified by $\mathrm{SiO}_{2}$ column chromatography concentrated, and further purified by recycling preparative HPLC (polystyrene/divinylbenzene stationary phase) with $\mathrm{CHCl}_{3}$. In cases where an excess of a compound is used, the unreacted portion is retrieved during column chromatography as its rf value is generally higher than the generated product.

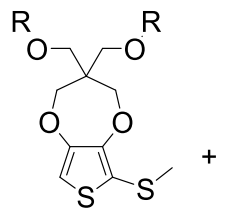

B

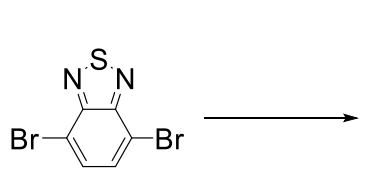

C

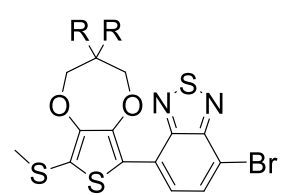

D

\section{Compound D}

The reaction was conducted according to the general procedure without modification using compound $\mathbf{B}$ ( $3.0 \mathrm{~g}$, $6.16 \mathrm{mmol}, 1.0$ equiv), compound $\mathbf{C}\left(5.44 \mathrm{~g}, 18.49 \mathrm{mmol}, 3.0\right.$ equiv) $\mathrm{Pd}(\mathrm{OAc})_{2}(4 \mathrm{~mol} \%, 55.3 \mathrm{mg}),(\mathrm{o}-\mathrm{MeOPh})_{3} \mathrm{P}$ $(8 \mathrm{~mol} \%, 173.6 \mathrm{mg})$, pivalic acid $\left(314.6 \mathrm{mg}, 3.08 \mathrm{mmol}, 0.5\right.$ equiv) and $\mathrm{Cs}_{2} \mathrm{CO}_{3}(3.01 \mathrm{~g}, 9.24 \mathrm{mmol}, 1.5 \mathrm{equiv})$ in $35 \mathrm{~mL}$ of anhydrous toluene. The product was filtered by column chromatography $\left(\mathrm{CH}_{2} \mathrm{Cl}_{2}\right.$ :hexanes, gradient of 1:1 to $2: 1)$ and purified by preparative HPLC to provide compound D (1.72 g, $40 \%$ yield) as an orange oil. Unreacted compound $\mathbf{C}$ and oligomer $\mathbf{3}$ was recovered in $\sim 20 \%$ yield. 
- $\quad{ }^{1} \mathrm{H}$ NMR (400 MHz, $\left.\mathrm{CDCl}_{3}\right) \delta 8.11(\mathrm{~d}, \mathrm{~J}=7.9 \mathrm{~Hz}, 1 \mathrm{H}), 7.79(\mathrm{~d}, \mathrm{~J}=7.9 \mathrm{~Hz}, 1 \mathrm{H}), 4.18(\mathrm{~d}, \mathrm{~J}=6.8 \mathrm{~Hz}, 4 \mathrm{H})$, $3.54(\mathrm{~s}, 4 \mathrm{H}), 3.30(\mathrm{~d}, \mathrm{~J}=5.7 \mathrm{~Hz}, 4 \mathrm{H}), 2.47(\mathrm{~s}, 3 \mathrm{H}), 1.48(\mathrm{dt}, \mathrm{J}=11.6,5.7 \mathrm{~Hz}, 2 \mathrm{H}), 1.41-1.23(\mathrm{~m}, 18 \mathrm{H}), 0.90$ $-0.84(\mathrm{~m}, 13 \mathrm{H})$.

- ${ }^{13} \mathrm{C}$ NMR $\left(101 \mathrm{MHz}, \mathrm{CDCl}_{3}\right) \delta 147.79,132.40,127.07,125.25,111.26,74.21,74.18,73.95,73.77,69.84$, 47.81, 39.53, 30.59, 30.56, 29.03, 23.92, 23.00, 20.33, 14.03, 11.08.

- $\quad H R M S(A P C I),[M+H]+m / z$ calcd. for $\mathrm{C}_{32} \mathrm{H}_{48} \mathrm{BrN}_{2} \mathrm{O}_{4} \mathrm{~S}_{3}: 699.1954$; found 699.1944.
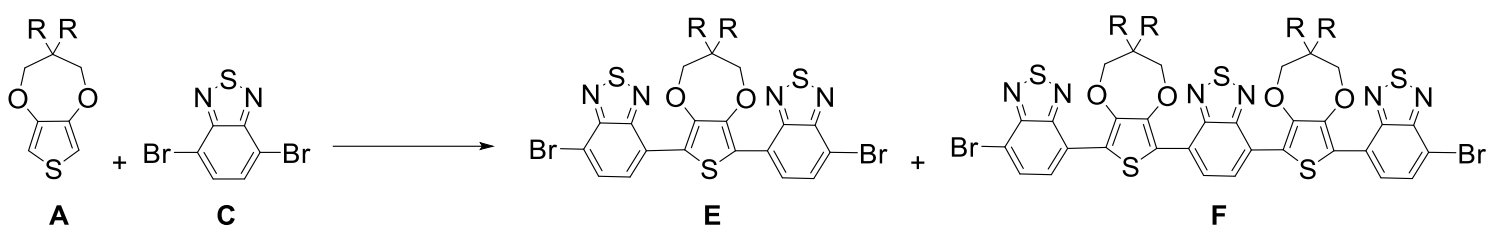

\section{Compounds E and F}

The reaction was conducted according to the general procedure using compound $\mathbf{A}(2.0 \mathrm{~g}, 4.55 \mathrm{mmol}, 1.0$ equiv), compound $\mathbf{C}$ (6.67 g, $22.7 \mathrm{mmol}, 5.0$ equiv) $\mathrm{Pd}(\mathrm{OAc})_{2}$ (4 mol \%, $\left.40.77 \mathrm{mg}\right)$, (o- $\left.\mathrm{MeOPh}\right)_{3} \mathrm{P}(8 \mathrm{~mol} \%, 127.98 \mathrm{mg})$, pivalic acid (927.34 mg, $9.08 \mathrm{mmol}, 2.0$ equiv) and $\mathrm{Cs}_{2} \mathrm{CO}_{3}(4.59 \mathrm{~g}, 14.07 \mathrm{mmol}, 3.1$ equiv) in $130 \mathrm{ml}$ anhydrous toluene. After work-up, the crude material was dissolved in hexane to precipitate out and recover excess compound $\mathbf{C}$ from the mixture and the filtrate was concentrated. The two products were purified by column chromatography $\left(\mathrm{CH}_{2} \mathrm{Cl}_{2}\right.$ :hexanes, gradient of $1: 1$ to $\left.3: 1\right)$ to sequentially provide compound $\mathbf{E}$ as a dark red solid (1.69 g, $43 \%$ yield) and compound $\mathbf{F}$ as a dark purple solid (624 $\mathrm{mg}, 10 \%$ yield).

\section{Compound $\mathbf{E}$}

- ${ }^{1} \mathrm{H}$ NMR $\left(400 \mathrm{MHz}, \mathrm{CDCl}_{3}\right) \delta 8.19(\mathrm{~d}, J=7.8 \mathrm{~Hz}, 2 \mathrm{H}), 7.85(\mathrm{~d}, J=7.9 \mathrm{~Hz}, 2 \mathrm{H}), 4.28(\mathrm{~s}, 4 \mathrm{H}), 3.60(\mathrm{~s}, 4 \mathrm{H})$, $3.34(\mathrm{~d}, J=5.7 \mathrm{~Hz}, 4 \mathrm{H}), 1.56-1.46(\mathrm{~m}, 1 \mathrm{H}), 1.44-1.22(\mathrm{~m}, 17 \mathrm{H}), 0.93-0.84(\mathrm{~m}, 12 \mathrm{H})$.

- $\quad{ }^{13} \mathrm{C}$ NMR $\left(101 \mathrm{MHz}, \mathrm{CDCl}_{3}\right) \delta 153.42,152.03,148.14,132.37,127.77,125.47,118.54,111.85,74.24,73.88$, 69.94, 47.78, 39.53, 30.57, 29.03, 23.92, 23.02, 14.04, 11.08.

- $\quad H R M S$ (APCI), $[\mathrm{M}+\mathrm{H}]^{+} \mathrm{m} / z$ calcd. for $\mathrm{C}_{37} \mathrm{H}_{47} \mathrm{Br}_{2} \mathrm{~N}_{2} \mathrm{O}_{4} \mathrm{~S}_{3}: 865.1121$; found 865.1186.

\section{Compound F}

- $\quad{ }^{1} \mathrm{H}$ NMR $\left(400 \mathrm{MHz}, \mathrm{CDCl}_{3}\right) \delta 8.43(\mathrm{~s}, 2 \mathrm{H}), 8.21(\mathrm{~d}, J=7.9 \mathrm{~Hz}, 2 \mathrm{H}), 7.87(\mathrm{~d}, J=7.9 \mathrm{~Hz}, 2 \mathrm{H}), 4.31(\mathrm{~d}, J=$ $7.7 \mathrm{~Hz}, 8 \mathrm{H}), 3.62(\mathrm{~s}, 8 \mathrm{H}), 3.35(\mathrm{~d}, J=5.7 \mathrm{~Hz}, 8 \mathrm{H}), 1.53(\mathrm{dt}, J=12.0,5.6 \mathrm{~Hz}, 6 \mathrm{H}), 1.42-1.24(\mathrm{~m}, 38 \mathrm{H}), 0.93$ $-0.87(\mathrm{~m}, 24 \mathrm{H})$.

- ${ }^{13} \mathrm{C}$ NMR $\left(101 \mathrm{MHz}, \mathrm{CDCl}_{3}\right) \delta 153.46,152.51,152.13,148.25,132.40,127.77,127.65,124.10,119.74$, $111.56,74.24,73.87,69.99,47.79,39.56,30.58,29.60,29.04,23.95,23.03,14.05,11.10$.

- $\quad$ HRMS (APCI), $[\mathrm{M}+\mathrm{H}]^{+} m / z$ calcd. for $\mathrm{C}_{68} \mathrm{H}_{91} \mathrm{Br}_{2} \mathrm{~N}_{6} \mathrm{O}_{8} \mathrm{~S}_{5}$ : 1437.3863; found 1437.3866.
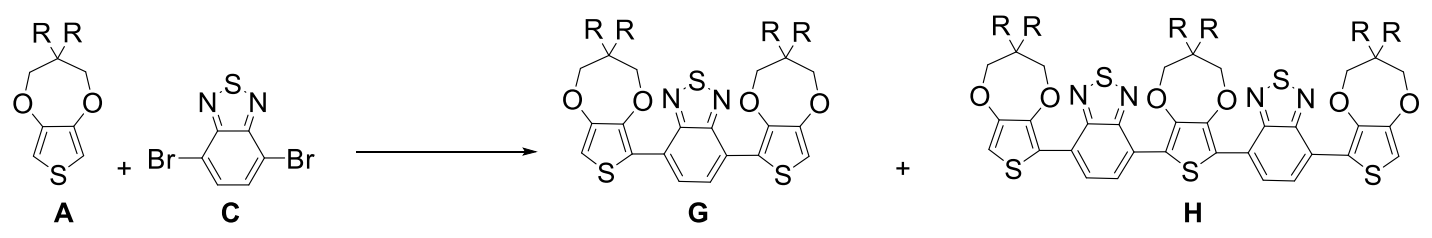

\section{Compound $\mathbf{G}$ and $H$}

The reaction was conducted according to the general procedure without modification using compound A (10.3 g, $23.46 \mathrm{mmol}, 6.0$ equiv), compound $\mathbf{C}\left(1.15 \mathrm{~g}, 3.91 \mathrm{mmol}, 1.0\right.$ equiv), $\mathrm{Pd}(\mathrm{OAc})_{2}(2 \mathrm{~mol} \%, 17.56 \mathrm{mg})$, (o- 
$\mathrm{MeOPh})_{3} \mathrm{P}(4 \mathrm{~mol} \%, 55.1 \mathrm{mg})$, pivalic acid (400 mg, $3.91 \mathrm{mmol}, 1.0$ equiv), and $\mathrm{Cs}_{2} \mathrm{CO}_{3}(3.7 \mathrm{~g}, 11.73 \mathrm{mmol}, 3.0$ equiv) in $75 \mathrm{ml}$ anhydrous toluene. The two products were purified by column chromatography $\left(\mathrm{CH}_{2} \mathrm{Cl}_{2}\right.$ :hexanes, gradient of 1:2 to 2:1) to sequentially provide compound $\mathbf{G}$ as an orange oil (1.86 g, $47 \%$ yield) and compound $\mathbf{H}$ as a sticky, magenta solid (1.74 g, $28 \%$ yield).

\section{Compound G}

- ${ }^{1} \mathrm{H}$ NMR $\left(400 \mathrm{MHz}, \mathrm{CDCl}_{3}\right) \delta 8.29(\mathrm{~s}, 2 \mathrm{H}), 6.66(\mathrm{~s}, 2 \mathrm{H}), 4.20(\mathrm{~s}, 4 \mathrm{H}), 4.10(\mathrm{~s}, 4 \mathrm{H}), 3.54(\mathrm{~s}, 8 \mathrm{H}), 3.31(\mathrm{~d}, \mathrm{~J}=$ $5.5 \mathrm{~Hz}, 8 \mathrm{H}), 1.56-1.45(\mathrm{~m}, 7 \mathrm{H}), 1.40-1.23(\mathrm{~m}, 42 \mathrm{H}), 0.95-0.84(\mathrm{~m}, 30 \mathrm{H})$.

- ${ }^{13} \mathrm{C}$ NMR $\left(101 \mathrm{MHz}, \mathrm{CDCl}_{3}\right) \delta 152.56,149.66,147.75,127.47,124.11,117.49,106.38,74.23,73.74,69.87$, 47.81, 39.54, 30.57, 29.03, 23.92, 23.01, 14.02, 11.07.

- $\quad$ HRMS (APCI), $[\mathrm{M}+\mathrm{H}]^{+} m / z$ calcd. for $\mathrm{C}_{56} \mathrm{H}_{88} \mathrm{~N}_{2} \mathrm{O}_{8} \mathrm{~S}_{3}$ : 1013.5776; found 1013.5771 .

\section{Compound H}

- $\quad{ }^{1} \mathrm{H}$ NMR $\left(400 \mathrm{MHz}, \mathrm{CDCl}_{3}\right) \delta 8.32(\mathrm{q}, J=8.0 \mathrm{~Hz}, 4 \mathrm{H}), 6.64(\mathrm{~s}, 2 \mathrm{H}), 4.38-4.03(\mathrm{~m}, 12 \mathrm{H}), 3.59(\mathrm{~d}, J=40.8$ $\mathrm{Hz}, 12 \mathrm{H}), 3.35(\mathrm{dd}, J=21.8,5.6 \mathrm{~Hz}, 12 \mathrm{H}), 1.53(\mathrm{tq}, J=14.5,8.5,7.0 \mathrm{~Hz}, 7 \mathrm{H}), 1.47-1.24(\mathrm{~m}, 51 \mathrm{H}), 1.00-$ $0.83(\mathrm{~m}, 38 \mathrm{H})$.

- ${ }^{13} \mathrm{C} \mathrm{NMR}\left(101 \mathrm{MHz}, \mathrm{CDCl}_{3}\right) \delta 152.45,152.41,149.57,147.89,147.74,127.27,127.10,124.00,123.95,119.20$, 117.63, 106.53, 77.28, 74.19, 74.13, 73.59, 69.97, 69.81, 47.80, 39.64, 39.60, 30.65, 29.08, 24.05, 24.00, 23.10, 23.07, 14.10, 11.17, 11.13 .

- $\quad H R M S$ (APCI), $[\mathrm{M}+\mathrm{H}]^{+} \mathrm{m} / z$ calcd. for $\mathrm{C}_{87} \mathrm{H}_{132} \mathrm{~N}_{4} \mathrm{O}_{12} \mathrm{~S}_{5}$ : 1585.8518 ; found 1585.8509.

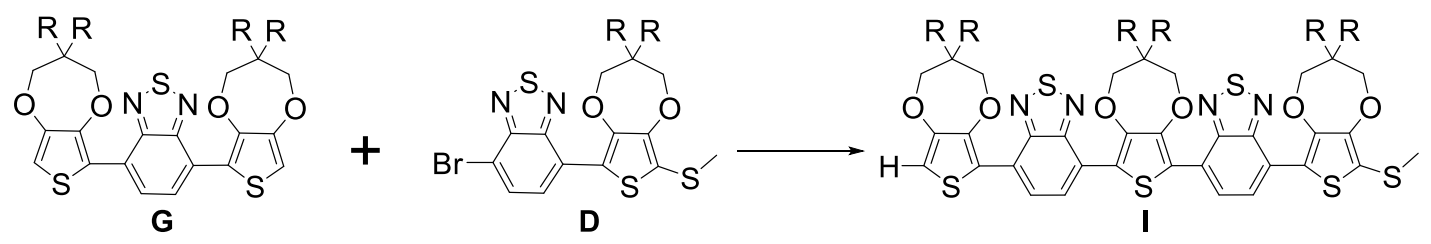

\section{Compound I}

The reaction was conducted according to the general procedure without modification using compound $\mathbf{G}$ (761.3 mg, 0.75 mmol, 2.7 equiv), compound $\mathbf{D}$ (194.7 mg, $0.28 \mathrm{mmol}, 1.0$ equiv), $\mathrm{Pd}(\mathrm{OAc})_{2}(8 \mathrm{~mol} \%, 5.0 \mathrm{mg})$, (o$\mathrm{MeOPh})_{3} \mathrm{P}(16 \mathrm{~mol} \%, 15.7 \mathrm{mg})$, pivalic acid (14.3 mg, $0.14 \mathrm{mmol}, 0.5$ equiv), and $\mathrm{Cs}_{2} \mathrm{CO}_{3}(135.9 \mathrm{mg}, 0.42 \mathrm{mmol}$, 1.5 equiv) in $7 \mathrm{ml}$ anhydrous toluene. The product was purified by column chromatography $\left(\mathrm{CH}_{2} \mathrm{Cl}_{2}\right.$ :hexanes, gradient of 1:3 to 1:1) to provide compound I as a sticky, magenta solid (265 $\mathrm{mg}, 58 \%$ yield).

- ${ }^{1} \mathrm{H}$ NMR $\left(400 \mathrm{MHz}, \mathrm{CDCl}_{3}\right) \delta 8.40-8.31(\mathrm{~m}, 4 \mathrm{H}), 6.66(\mathrm{~s}, 1 \mathrm{H}), 4.32(\mathrm{~s}, 5 \mathrm{H}), 4.27-4.17(\mathrm{~m}, 6 \mathrm{H}), 4.10(\mathrm{~s}, 2 \mathrm{H})$, $3.69-3.51(\mathrm{~m}, 13 \mathrm{H}), 3.35(\mathrm{dd}, \mathrm{J}=16.2,5.7 \mathrm{~Hz}, 13 \mathrm{H}), 2.49(\mathrm{~s}, 3 \mathrm{H}), 1.52(\mathrm{dt}, \mathrm{J}=11.7,5.9 \mathrm{~Hz}, 7 \mathrm{H}), 1.46-1.22$ $(\mathrm{m}, 56 \mathrm{H}), 0.91(\mathrm{~m}, 40 \mathrm{H})$.

- ${ }^{13} \mathrm{C}$ NMR $\left(101 \mathrm{MHz}, \mathrm{CDCl}_{3}\right) \delta 152.47,150.00,149.63,148.00,147.94,147.80,147.46,127.52,127.26,127.16$, 124.16, 124.03, 123.96, 123.57, 119.21, 119.06, 118.27, 117.58, 116.42, 106.55, 74.22, 74.16, 73.94, 73.68, 70.00, 69.85, 47.82, 39.59, 30.62, 29.06, 23.98, 23.05, 20.51, 14.07, 11.13.

- $\quad$ HRMS (APCI), $[\mathrm{M}+\mathrm{H}]^{+} \mathrm{m} / z$ calcd. for $\mathrm{C}_{88} \mathrm{H}_{135} \mathrm{~N}_{4} \mathrm{O}_{12} \mathrm{~S}_{6}$ : 1631.8395 ; found 1631.8355 . 


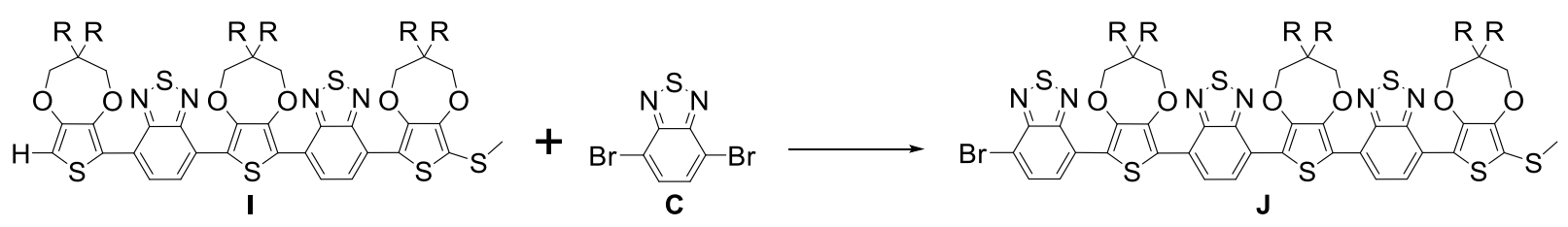

\section{Compound J}

The reaction was conducted according to the general procedure using compound I (177.1 $\mathrm{mg}, 0.108 \mathrm{mmol}, 1.0$ equiv), compound $\mathbf{C}$ (127.5 mg, $0.434 \mathrm{mmol}, 4.0$ equiv) $\mathrm{Pd}(\mathrm{OAc})_{2}(8 \mathrm{~mol} \%, 2 \mathrm{mg})$, (o-MeOPh) 3 P (16 mol \%, 6.1 $\mathrm{mg}$ ), pivalic acid (5.5 mg, $0.054 \mathrm{mmol}, 0.5$ equiv), and $\mathrm{Cs}_{2} \mathrm{CO}_{3}(54.6 \mathrm{mg}, 0.173 \mathrm{mmol}, 1.6$ equiv) in $3 \mathrm{ml}$ anhydrous toluene. After work-up, the crude material was dissolved in hexane to precipitate out and recover excess compound $\mathbf{C}$ from the mixture and the filtrate was concentrated. The product was purified by column chromatography $\left(\mathrm{CH}_{2} \mathrm{Cl}_{2}\right.$ :hexanes, gradient of 1:1 to 3:1) to provide compound $\mathbf{J}$ as a dark purple solid (93.9 $\mathrm{mg}$, $47 \%$ yield).

- ${ }^{1} \mathrm{H}$ NMR $\left(400 \mathrm{MHz}, \mathrm{CDCl}_{3}\right) \delta 8.43-8.31(\mathrm{~m}, 4 \mathrm{H}), 8.18(\mathrm{~d}, \mathrm{~J}=7.9 \mathrm{~Hz}, 1 \mathrm{H}), 7.83(\mathrm{~d}, \mathrm{~J}=7.9 \mathrm{~Hz}, 1 \mathrm{H}), 4.38-$ $4.25(\mathrm{~m}, 8 \mathrm{H}), 4.21(\mathrm{~d}, \mathrm{~J}=13.5 \mathrm{~Hz}, 4 \mathrm{H}), 3.69-3.51(\mathrm{~m}, 12 \mathrm{H}), 3.42-3.28(\mathrm{~m}, 12 \mathrm{H}), 2.49(\mathrm{~s}, 3 \mathrm{H}), 1.57-1.47$ $(\mathrm{m}, 7 \mathrm{H}), 1.43-1.24(\mathrm{~m}, 57 \mathrm{H}), 0.96-0.84(\mathrm{~m}, 39 \mathrm{H})$.

- ${ }^{13} \mathrm{C}$ NMR $\left(101 \mathrm{MHz}, \mathrm{CDCl}_{3}\right) \delta 153.51,152.58,152.51,152.19,150.08,148.30,148.17,148.06,147.98,147.55$, $132.44,127.91,127.81,127.69,127.28,125.81,124.45,124.05,123.89,123.79,119.90,119.30,118.25$, $117.95,116.51,111.57,74.28,74.23,74.03,73.90,73.80,70.06,69.94,53.34,47.87,47.84,39.62,39.60$, $30.62,29.64,29.07,23.98,23.96,23.06,20.57,14.08,11.13$.

- $\quad H R M S$ (APCI), [M+H] $]^{+} m / z$ calcd. for $\mathrm{C}_{94} \mathrm{H}_{136} \mathrm{BrN}_{6} \mathrm{O}_{12} \mathrm{~S}_{7}$ : 1843.7439 ; found 1843.7404 .
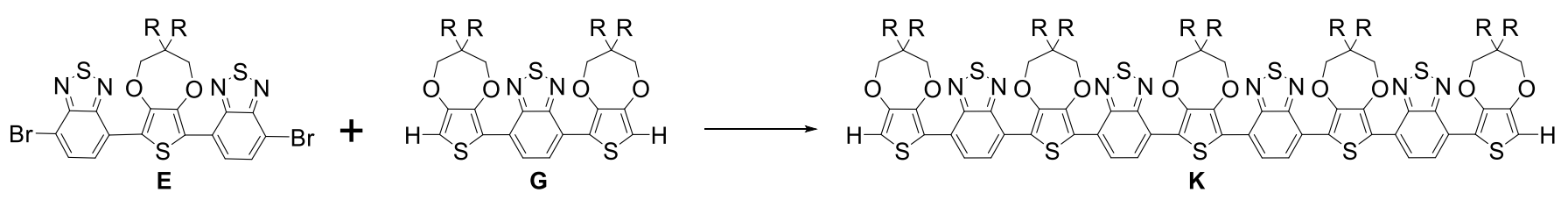

\section{Compound K}

The reaction was conducted according to the general procedure using compound $\mathbf{E}$ (950.6 $\mathrm{mg}, 0.938 \mathrm{mmol}, 4.0$ equiv), compound $\mathbf{G}$ (203.2 mg, $0.234 \mathrm{mmol}, 1.0$ equiv) $\mathrm{Pd}(\mathrm{OAc})_{2}(8 \mathrm{~mol} \%, 4.2 \mathrm{mg}),(\mathrm{o}-\mathrm{MeOPh})_{3} \mathrm{P}(16 \mathrm{~mol} \%$, $13.2 \mathrm{mg}$ ), pivalic acid (24 mg, $0.234 \mathrm{mmol}, 1.0$ equiv) and $\mathrm{Cs}_{2} \mathrm{CO}_{3}(229.1 \mathrm{mg}, 0.725 \mathrm{mmol}, 3.1$ equiv) in $3 \mathrm{ml}$ anhydrous toluene. The product was purified by column chromatography $\left(\mathrm{CH}_{2} \mathrm{Cl}_{2}\right.$ :hexanes, gradient of 1:2 to 2:1) to provide compound $\mathbf{K}$ as a dark blue solid $(233 \mathrm{mg}, 37 \%$ yield).

- ${ }^{1} \mathrm{H}$ NMR $\left(500 \mathrm{MHz}, \mathrm{CDCl}_{3}, \mathrm{ppm}\right) \delta 8.48(\mathrm{~s}, 4 \mathrm{H}), 8.45(\mathrm{~d}, \mathrm{~J}=7.9 \mathrm{~Hz}, 2 \mathrm{H}), 8.40(\mathrm{~d}, \mathrm{~J}=7.9 \mathrm{~Hz}, 2 \mathrm{H}), 6.74(\mathrm{~s}$, 2H), $4.40(\mathrm{~d}, \mathrm{~J}=3.9 \mathrm{~Hz}, 13 \mathrm{H}), 4.28(\mathrm{~s}, 4 \mathrm{H}), 4.17(\mathrm{~s}, 4 \mathrm{H}), 3.71(\mathrm{~s}, 13 \mathrm{H}), 3.61(\mathrm{~s}, 9 \mathrm{H}), 3.43(\mathrm{~d}, \mathrm{~J}=5.7 \mathrm{~Hz}, 12 \mathrm{H})$, $3.38(\mathrm{~d}, \mathrm{~J}=5.6 \mathrm{~Hz}, 8 \mathrm{H}), 1.60(\mathrm{dt}, \mathrm{J}=19.2,6.0 \mathrm{~Hz}, 12 \mathrm{H}), 1.53-1.29(\mathrm{~m}, 91 \mathrm{H}), 1.04-0.91(\mathrm{~m}, 67 \mathrm{H})$.

- $\quad{ }^{13} \mathrm{C}$ NMR (126 MHz, $\left.\mathrm{CDCl}_{3}, \mathrm{ppm}\right) \delta 152.74,152.70,149.81,148.19,148.14,147.95,127.74,127.67,127.49$, $124.32,124.30,124.23,119.45,119.38,119.34,117.76,106.65,74.40,74.34,73.94,73.85,70.17,69.99,47.96$, 39.76, 39.72, 30.77, 30.74, 29.21, 24.15, 24.10, 23.20, 23.18, 14.22, 14.19, 11.28, 11.24.

- $\quad H R M S$ (APCI), [M+H] ${ }^{+} \mathrm{m} / z$ calcd. for $\mathrm{C}_{149} \mathrm{H}_{221} \mathrm{~N}_{8} \mathrm{O}_{20} \mathrm{~S}_{9}: 2730.4003$; found 2730.3894 . 


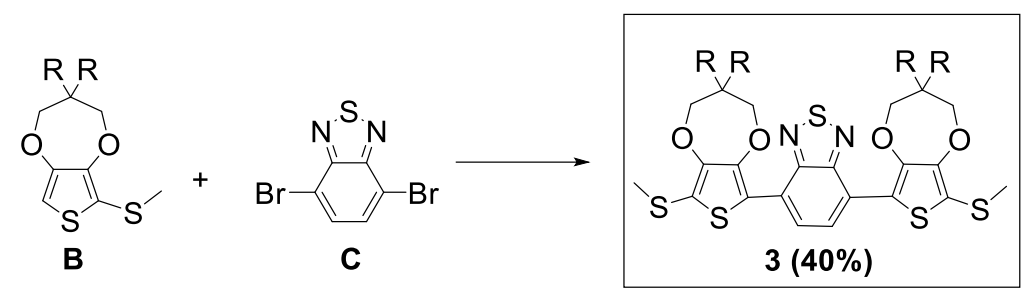

\section{Oligomer $\mathbf{n}=\mathbf{3}$}

The reaction was conducted according to the general procedure without modification using compound B (1.95 g, $4.01 \mathrm{mmol}, 2.1$ equiv) and compound $\mathbf{C}\left(560.8 \mathrm{mg}, 1.907 \mathrm{mmol}, 1.0\right.$ equiv), $\mathrm{Pd}(\mathrm{OAc})_{2}(5 \mathrm{~mol} \%, 5.0 \mathrm{mg})$, (o$\mathrm{MeOPh})_{3} \mathrm{P}(10 \mathrm{~mol} \%, 67.2 \mathrm{mg})$, pivalic acid $\left(97.4 \mathrm{mg}, 0.95 \mathrm{mmol}, 0.5\right.$ equiv) and $\mathrm{Cs}_{2} \mathrm{CO}_{3}(932 \mathrm{mg}, 2.86 \mathrm{mmol}$, 1.5 equiv) in $5 \mathrm{ml}$ anhydrous toluene. The product was purified by column chromatography $\left(\mathrm{CH}_{2} \mathrm{Cl}_{2}\right.$ :hexanes, $\left.1: 3\right)$ and further purified by preparative HPLC to provide oligomer $\mathbf{n}=\mathbf{3}$ as an orange viscous oil ( $886 \mathrm{mg}, 42 \%$ yield).

- $\quad{ }^{1} \mathrm{H}$ NMR $\left(500 \mathrm{MHz}, \mathrm{CD}_{2} \mathrm{Cl}_{2}, \mathrm{ppm}\right) \delta 8.33(\mathrm{~s}, 2 \mathrm{H}), 4.21(\mathrm{~s}, 4 \mathrm{H}), 4.15(\mathrm{~s}, 4 \mathrm{H}), 3.54(\mathrm{~s}, 8 \mathrm{H}), 3.32(\mathrm{dd}, \mathrm{J}=5.0 \mathrm{~Hz}$, $8 \mathrm{H}), 2.45(\mathrm{~s}, 6 \mathrm{H}), 1.43-1.27(\mathrm{~m}, 34 \mathrm{H}), 0.87(\mathrm{~m}, 22 \mathrm{H})$.

- ${ }^{13} \mathrm{C}$ NMR $\left(126 \mathrm{MHz}, \mathrm{CD}_{2} \mathrm{Cl}_{2}, \mathrm{ppm}\right) \delta 151.92,149.69,147.47,126.69,122.98,117.32,115.89,109.21,73.48$, $73.38,73.20,69.04,54.33,54.11,53.89,53.68,53.46,47.58,39.24,30.32,28.91,23.60,23.55,23.26,20.32$, $14.23,10.94$.

- $\quad$ HRMS (ESI), [M+H]+ m/z calcd. for $\mathrm{C}_{58} \mathrm{H}_{92} \mathrm{~N}_{2} \mathrm{O}_{8} \mathrm{~S}_{5}$ : 1104.5458; found 1104.5441 .
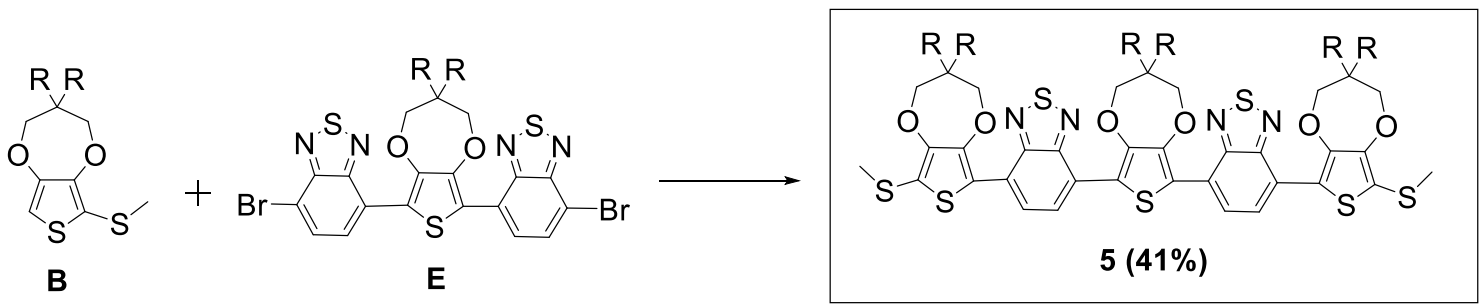

\section{Oligomer $\mathbf{n}=\mathbf{5}$}

The reaction was conducted according to the general procedure without modification using compound B ( $288 \mathrm{mg}$, $0.592 \mathrm{mmol}, 2.2$ equiv) and compound $\mathbf{E}$ (233.2 mg, $0.269 \mathrm{mmol}, 1.0$ equiv), $\mathrm{Pd}(\mathrm{OAc}) 2$ (8 mol \%, $6.3 \mathrm{mg}),(\mathrm{o}-$ $\mathrm{MeOPh})_{3} \mathrm{P}(16 \mathrm{~mol} \%, 15.2 \mathrm{mg})$, pivalic acid (13.7 mg, $0.135 \mathrm{mmol}, 0.5$ equiv), and $\mathrm{Cs}_{2} \mathrm{CO}_{3}(131.5 \mathrm{mg}, 0.404$ mmol, 1.5 equiv) in $2 \mathrm{ml}$ anhydrous toluene. The product was purified by column chromatography $\left(\mathrm{CH}_{2} \mathrm{Cl}_{2}\right.$ :hexanes, 1:1) and further purified by preparative HPLC to provide oligomer $\mathbf{n}=\mathbf{5}$ as a magenta wax-like solid (185 mg, 41\% yield).

- $\quad{ }^{1} \mathrm{H}$ NMR (400 MHz, $\left.\mathrm{CDCl}_{3}, \mathrm{ppm}\right) \delta 8.37(\mathrm{q}, \mathrm{J}=8.0 \mathrm{~Hz}, 4 \mathrm{H}), 4.31(\mathrm{~s}, 4 \mathrm{H}), 4.21(\mathrm{~d}, \mathrm{~J}=12.6 \mathrm{~Hz}, 8 \mathrm{H}), 3.63(\mathrm{~s}$, $4 \mathrm{H}), 3.57(\mathrm{~d}, \mathrm{~J}=2.4 \mathrm{~Hz}, 9 \mathrm{H}), 3.34(\mathrm{dd}, \mathrm{J}=11.8,5.6 \mathrm{~Hz}, 12 \mathrm{H}), 2.49(\mathrm{~s}, 6 \mathrm{H}), 1.58-1.46(\mathrm{~m}, 7 \mathrm{H}), 1.46-1.37$ (m, 5H), $1.37-1.32(\mathrm{~m}, 13 \mathrm{H}), 1.32-1.23(\mathrm{~m}, 36 \mathrm{H}), 0.90(\mathrm{dd}, \mathrm{J}=7.3,4.9 \mathrm{~Hz}, 39 \mathrm{H})$.

- $\quad{ }^{13} \mathrm{C}$ NMR $\left(101 \mathrm{MHz}, \mathrm{CDCl}_{3}, \mathrm{ppm}\right) \delta 150.04,148.01,147.49,127.70,127.23,123.69,119.09,74.23,73.99$, 73.74, 70.01, 69.89, 47.83, 39.57, 30.59, 29.04, 23.95, 23.03, 20.54, 14.05, 11.11 .

- $\quad H R M S$ (APCI), $[\mathrm{M}+\mathrm{H}]^{+} m / z$ calcd. for $\mathrm{C}_{89} \mathrm{H}_{137} \mathrm{~N}_{4} \mathrm{O}_{12} \mathrm{~S}_{7}$ : 1677.8272; found 1677.8253. 


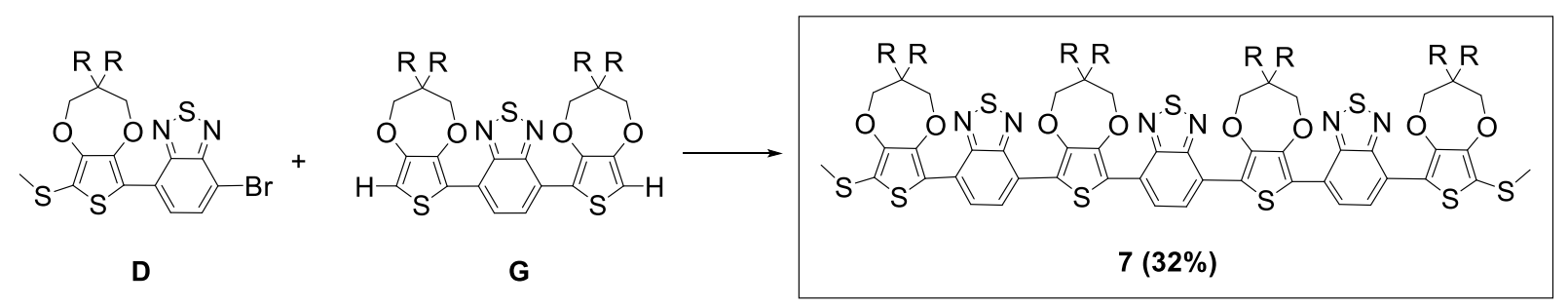

\section{Oligomer $\mathrm{n}=7$}

The reaction was conducted according to the general procedure without modification using compound D (445.9 $\mathrm{mg}, 0.637 \mathrm{mmol}, 2.7$ equiv) and compound $\mathbf{G}$ (239.2 mg, $0.236 \mathrm{mmol}, 1.0$ equiv), $\mathrm{Pd}(\mathrm{OAc}) 2$ (4 mol \%, $2.7 \mathrm{mg}$ ), $(\mathrm{o}-\mathrm{MeOPh})_{3} \mathrm{P}(8 \mathrm{~mol} \%, 6.7 \mathrm{mg})$, pivalic acid $\left(24.1 \mathrm{mg}, 0.236 \mathrm{mmol}, 1.0\right.$ equiv) and $\mathrm{Cs}_{2} \mathrm{CO}_{3}(238.4 \mathrm{mg}, 0.732$ mmol, 3.1 equiv) in $3 \mathrm{ml}$ anhydrous toluene. The product was purified by column chromatography $\left(\mathrm{CH}_{2} \mathrm{Cl}_{2}\right.$ :hexanes, gradient of 1:1 to 2:1) and further purified by preparative HPLC to provide oligomer 7-A as a purple solid (170 mg, $32 \%$ yield).

- $\quad{ }^{1} \mathrm{H}$ NMR $\left(500 \mathrm{MHz}, \mathrm{CDCl}_{3}, \mathrm{ppm}\right) \delta 8.40(\mathrm{~s}, 3 \mathrm{H}), 8.40-8.32(\mathrm{~m}, 4 \mathrm{H}), 4.33(\mathrm{~s}, 8 \mathrm{H}), 4.21(\mathrm{~d}, J=17.4 \mathrm{~Hz}, 8 \mathrm{H})$, $3.57(\mathrm{~s}, 8 \mathrm{H}), 3.37(\mathrm{~d}, J=5.7 \mathrm{~Hz}, 8 \mathrm{H}), 3.32(\mathrm{~d}, J=5.8 \mathrm{~Hz}, 8 \mathrm{H}), 2.50(\mathrm{~s}, 6 \mathrm{H}), 1.53(\mathrm{~s}, 8 \mathrm{H}), 1.42-1.27(\mathrm{~m}, 67 \mathrm{H})$, $0.95-0.87(\mathrm{~m}, 52 \mathrm{H})$.

- $\quad{ }^{13} \mathrm{C}$ NMR $\left(201 \mathrm{MHz}, \mathrm{CDCl}_{3}, \mathrm{ppm}\right) \delta 152.47,152.43,150.04,148.05,148.01,147.46,127.21,124.12,124.01$, $123.49,119.39,119.17,118.32,116.35,74.25,74.18,73.89,73.71,69.97,69.77,47.85,47.82,39.66,39.62$, 30.66, 30.64, 29.10, 24.05, 24.01, 23.99, 23.11, 23.08, 20.56, 14.14, 14.11, 11.19, 11.16.

- $\quad \operatorname{HRMS}(\mathrm{APCI}),[\mathrm{M}+\mathrm{H}]^{+} \mathrm{m} / z$ calcd. for $\mathrm{C}_{120} \mathrm{H}_{181} \mathrm{~N}_{6} \mathrm{O}_{16} \mathrm{~S}_{9}: 2250.1015$; found 2250.1029 .

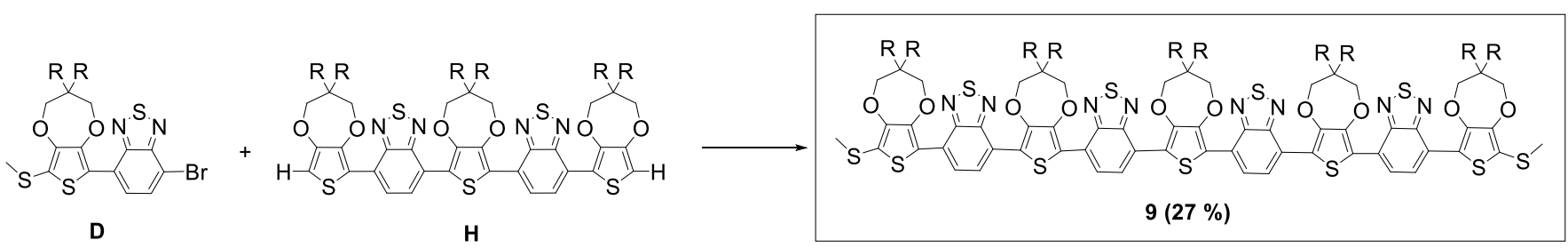

\section{Oligomer $\mathbf{n}=9$}

The reaction was conducted according to the general procedure without modification using compound D (135 mg, $0.277 \mathrm{mmol}, 2.2$ equiv) and compound $\mathbf{H}\left(200 \mathrm{mg}, 0.126 \mathrm{mmol}, 1.0\right.$ equiv), $\mathrm{Pd}(\mathrm{OAc})_{2}(8 \mathrm{~mol} \%, 3.0 \mathrm{mg}),(\mathrm{o}-$ $\mathrm{MeOPh})_{3} \mathrm{P}(16 \mathrm{~mol} \%, 7.1 \mathrm{mg})$, pivalic acid (12.9 mg, $0.158 \mathrm{mmol}, 1.0$ equiv) and $\mathrm{Cs}_{2} \mathrm{CO}_{3}(127.4 \mathrm{mg}, 0.391 \mathrm{mmol}$, 3.1 equiv) in $2 \mathrm{ml}$ anhydrous toluene. The product was purified by column chromatography $\left(\mathrm{CH}_{2} \mathrm{Cl}_{2}\right.$ :hexanes, gradient of 2:1 to 4:1) to provide oligomer $\mathbf{n = 9}$ as a blue solid (96 mg, $27 \%$ yield).

- $\quad{ }^{1} \mathrm{H}$ NMR $\left(800 \mathrm{MHz}, \mathrm{CDCl}_{3}, \mathrm{ppm}\right) \delta 8.45-8.39(\mathrm{~m}, 6 \mathrm{H}), 8.36(\mathrm{~d}, J=7.7 \mathrm{~Hz}, 2 \mathrm{H}), 4.35(\mathrm{~s}, 10 \mathrm{H}), 4.24(\mathrm{~s}, 4 \mathrm{H})$, $4.21(\mathrm{~s}, 4 \mathrm{H}), 3.67(\mathrm{~s}, 11 \mathrm{H}), 3.58(\mathrm{~s}, 8 \mathrm{H}), 3.39(\mathrm{~s}, 12 \mathrm{H}), 3.34(\mathrm{~s}, 8 \mathrm{H}), 2.52(\mathrm{~s}, 6 \mathrm{H}), 1.55(\mathrm{dd}, J=38.4,5.9 \mathrm{~Hz}$, $12 \mathrm{H}), 1.33(\mathrm{~d}, J=20.7 \mathrm{~Hz}, 88 \mathrm{H}), 0.97-0.89(\mathrm{~m}, 70 \mathrm{H})$.

- ${ }^{13} \mathrm{C}$ NMR (201 MHz, $\left.\mathrm{CDCl}_{3}, \mathrm{ppm}\right) \delta 152.59,152.53,152.48,150.07,148.08,148.05,147.49,127.53,127.26$, 124.18, 124.14, 124.07, 123.61, 119.37, 119.34, 119.17, 118.32, 116.40, 74.26, 74.21, 73.96, 73.81, 73.70, $70.03,69.98,69.86,47.86,47.83,39.65,39.61,34.61,31.53,30.65,30.63,29.10,25.22,24.03,24.00,23.98$, 23.10, 23.07, 22.60, 20.57, 14.12, 14.09, 14.06, 11.17, 11.14 .

- $\quad$ HRMS (APCI), $[\mathrm{M}+\mathrm{H}]^{+} \mathrm{m} / z$ calcd. for $\mathrm{C}_{151} \mathrm{H}_{225} \mathrm{~N}_{8} \mathrm{O}_{20} \mathrm{~S}_{11}: 2822.3757$; found 2822.3812 . 

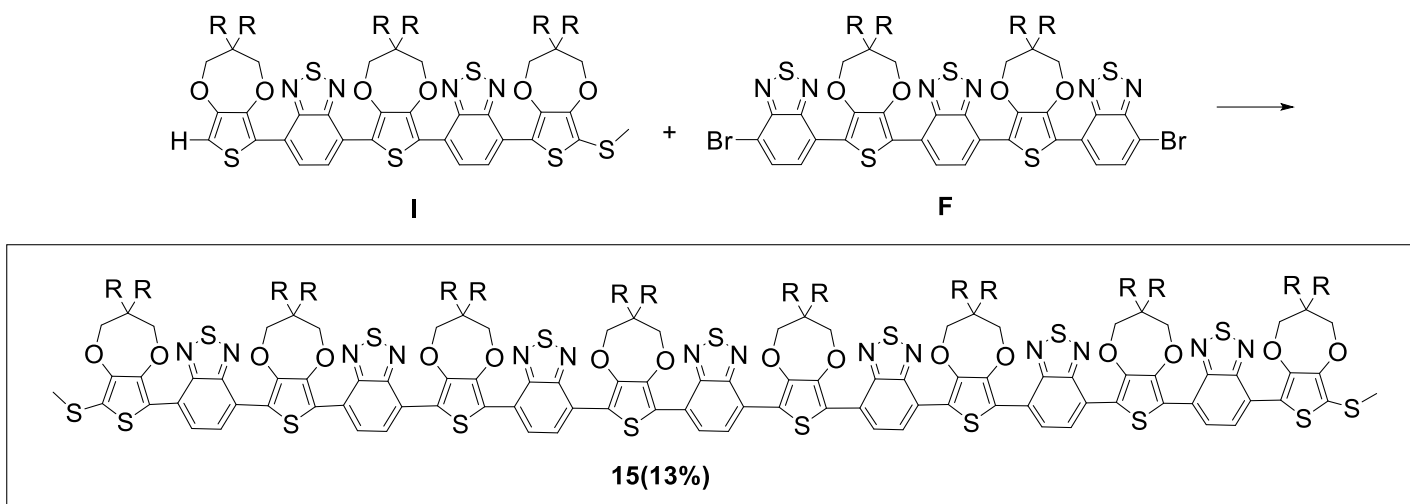

\section{Oligomer $\mathrm{n}=15$}

The reaction was conducted according to the general procedure without modification using compound I (264.9 mg, $0.162 \mathrm{mmol}, 2.3$ equiv) and compound $\mathbf{F}$ (101.5 mg, $0.071 \mathrm{mmol}, 1.0$ equiv), $\mathrm{Pd}(\mathrm{OAc})_{2}(20 \mathrm{~mol} \%, 4.2 \mathrm{mg}),(\mathrm{o}-$ $\mathrm{MeOPh})_{3} \mathrm{P}(40 \mathrm{~mol} \%, 10 \mathrm{mg})$, pivalic acid $\left(8.0 \mathrm{mg}, 0.078 \mathrm{mmol}, 1.1\right.$ equiv), and $\mathrm{Cs}_{2} \mathrm{CO}_{3}(71.7 \mathrm{mg}, 0.220 \mathrm{mmol}$, 3.1 equiv) in $3 \mathrm{ml}$ anhydrous toluene. The product was purified by column chromatography $\left(\mathrm{CH}_{2} \mathrm{Cl}_{2}\right.$ :hexanes, gradient of $1: 1$ to 5:1) and further purified by preparative HPLC to provide oligomer $\mathbf{n = 1 5}$ as a blue solid (42 $\mathrm{mg}$, $13 \%$ yield).

- $\quad{ }^{1} \mathrm{H}$ NMR $\left(800 \mathrm{MHz}, \mathrm{CDCl}_{3}, \mathrm{ppm}\right) \delta 8.47(\mathrm{~d}, J=8.6 \mathrm{~Hz}, 10 \mathrm{H}), 8.43-8.36(\mathrm{~m}, 4 \mathrm{H}), 4.36(\mathrm{t}, J=8.0 \mathrm{~Hz}, 24 \mathrm{H})$, $4.25(\mathrm{~s}, 4 \mathrm{H}), 4.22(\mathrm{~s}, 4 \mathrm{H}), 3.67(\mathrm{~d}, J=10.4 \mathrm{~Hz}, 24 \mathrm{H}), 3.60(\mathrm{~s}, 8 \mathrm{H}), 3.40-3.38(\mathrm{~m}, 24 \mathrm{H}), 3.34(\mathrm{~d}, J=5.6 \mathrm{~Hz}$, $8 \mathrm{H}), 2.51(\mathrm{~s}, 6 \mathrm{H}), 1.59-1.51(\mathrm{~m}, 20 \mathrm{H}), 1.45-1.28(\mathrm{~m}, 160 \mathrm{H}), 0.96-0.89(\mathrm{~m}, 118 \mathrm{H})$.

- ${ }^{13} \mathrm{C}$ NMR $\left(201 \mathrm{MHz}, \mathrm{CDCl}_{3}, \mathrm{ppm}\right) \delta 152.66,152.58,152.51,150.08,148.11,148.08,147.51,127.68,127.30$, 124.21, 124.13, 123.71, 119.35, 119.31, 119.14, 118.30, 116.43, 74.28, 74.23, 74.03, 73.89, 73.78, 70.08, 69.93, 47.88, 47.85, 39.63, 39.60, 30.64, 29.09, 24.01, 23.97, 23.09, 23.06, 20.59, 14.11, 14.08, 11.16, 11.13.

- $\quad$ MS (MALDI+), $[\mathrm{M}+\mathrm{H}] \mathrm{m} / z$ calcd. for $\mathrm{C}_{244} \mathrm{H}_{356} \mathrm{~N}_{14} \mathrm{O}_{32} \mathrm{~S}_{17}$ : 4541.20; found 4536.57 .
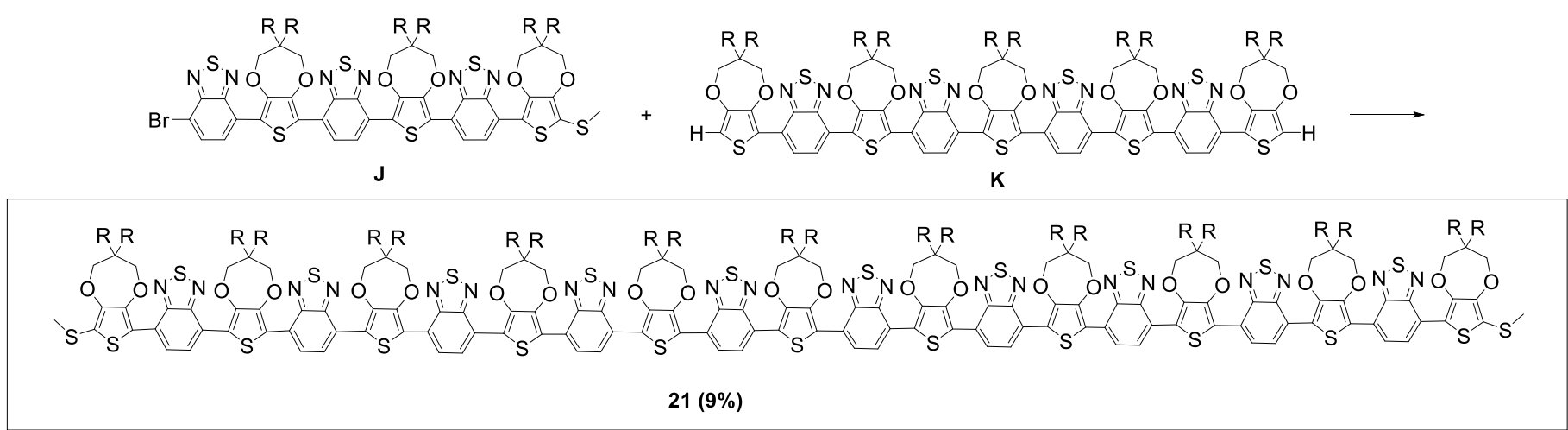

\section{Oligomer $\mathbf{n}=21$}

The reaction was conducted according to the general procedure without modification using compound 10 (93.9 $\mathrm{mg}, 0.051 \mathrm{mmol}, 2.2$ equiv) and compound 11 (63.2 $\mathrm{mg}, 0.023 \mathrm{mmol}, 1.0$ equiv), $\mathrm{Pd}(\mathrm{OAc})_{2}(40 \mathrm{~mol} \%, 2.7 \mathrm{mg})$, (o-MeOPh) $)_{3} \mathrm{P}\left(80 \mathrm{~mol} \%, 6.5 \mathrm{mg}\right.$ ), pivalic acid ( $2.3 \mathrm{mg}, 0.023 \mathrm{mmol}, 1.0$ equiv) and $\mathrm{Cs}_{2} \mathrm{CO}_{3}(23.4 \mathrm{mg}, 0.072 \mathrm{mmol}$, 3.1 equiv) in $2 \mathrm{ml}$ anhydrous toluene. The product was purified by column chromatography $\left(\mathrm{CH}_{2} \mathrm{Cl}_{2}\right.$ :hexanes, gradient of $1: 1$ to 5:1) and further purified by preparative HPLC to provide oligomer 21-A as a blue solid (37 mg, $26 \%$ yield). 
- $\quad{ }^{1} \mathrm{H} \mathrm{NMR}\left(800 \mathrm{MHz}, \mathrm{CDCl}_{3}\right) \delta 8.47(\mathrm{~s}, 16 \mathrm{H}), 8.42-8.35(\mathrm{~m}, 4 \mathrm{H}), 4.39(\mathrm{~s}, 46 \mathrm{H}), 4.24(\mathrm{~d}, J=25.9 \mathrm{~Hz}, 8 \mathrm{H}), 3.70$ (s, 48H), $3.60(\mathrm{~s}, 14 \mathrm{H}), 3.41(\mathrm{~s}, 52 \mathrm{H}), 3.35(\mathrm{~s}, 16 \mathrm{H}), 2.51(\mathrm{~d}, J=7.5 \mathrm{~Hz}, 6 \mathrm{H}), 1.56(\mathrm{~d}, J=42.1 \mathrm{~Hz}, 39 \mathrm{H}), 1.35$ $(\mathrm{s}, 309 \mathrm{H}), 0.94(\mathrm{~d}, J=8.0 \mathrm{~Hz}, 229 \mathrm{H})$.

- $\quad{ }^{13} \mathrm{C}$ NMR $\left(201 \mathrm{MHz}, \mathrm{CDCl}_{3}\right) \delta 152.57,150.03,148.02,147.45,127.56,124.12,119.36,119.12,118.30,116.42$, 74.27, 74.23, 73.90, 70.14, 69.94, 47.83, 39.65, 39.61, 30.65, 29.64, 29.10, 24.03, 23.99, 23.97, 23.10, 23.07, 20.58, 14.11, 14.09, 11.16, 11.13 .

- $\quad$ MS (MALDI+), $[\mathrm{M}+\mathrm{H}] \mathrm{m} / \mathrm{z}$ calcd. for $\mathrm{C}_{337} \mathrm{H}_{488} \mathrm{~N}_{20} \mathrm{O}_{44} \mathrm{~S}_{23}$ : 6261.09; found 6260.57.

\section{General Polymerization Condition}

To a Schlenk tube charged with a stir bar, compound A (1.0 equiv), compound B ( 0.05 equiv.), compound $\mathbf{C}$ (1.0 equiv), $\mathrm{Pd}(\mathrm{OAc})_{2}(2 \mathrm{~mol} \%),(\mathrm{o}-\mathrm{MeOPh})_{3} \mathrm{P}(4 \mathrm{~mol} \%)$, pivalic acid (1.1 equiv) and $\mathrm{Cs}_{2} \mathrm{CO}_{3}$ (3.1 equiv) were added, vacuumed for 30 minutes, and then subjected to $\mathrm{N}_{2}$ purge cycles three times. Anhydrous toluene ( $3 \mathrm{ml}$ ) was added via needle and syringe. The reaction was heated at $110^{\circ} \mathrm{C}$ in an oil bath until completion. After polymerization was complete, the mixture was precipitated into methanol. The solids were collected by a Soxhlet thimble and purified by Soxhlet extraction successively with methanol, acetone, hexane, dichloromethane and chloroform. 3 separate reaction were carried out with variations in heating time; $24 \mathrm{~h}$ ( $\mathrm{P} 1$, extracted from DCM fraction), $48 \mathrm{~h}$ ( $\mathrm{P} 2$, extracted form chloroform extract), and $72 \mathrm{~h}$ ( $\mathrm{P} 3$, extracted from chloroform extract). The fractions were concentrated and precipitated into methanol. The polymer was collected and further purified by recycling HPLC.

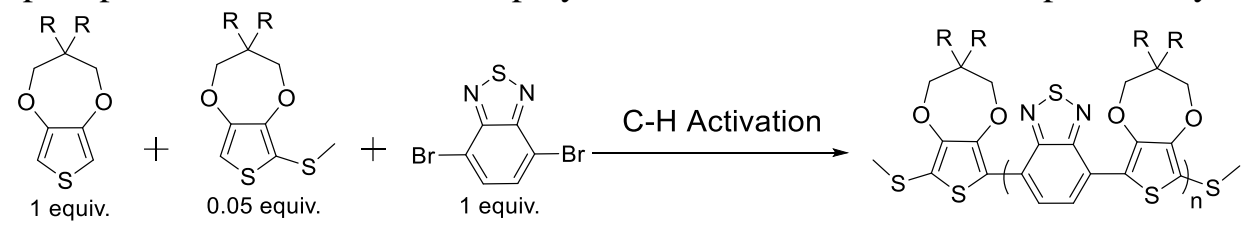

\begin{tabular}{|c|c|c|c|c|}
\hline Polymer & Reaction Time & Yield & GPC Mn (Da) & PDI \\
\hline P1 & $24 \mathrm{~h}$ & $112 \mathrm{mg}$ & 9,396 & 1.37 \\
\hline P2 & $48 \mathrm{~h}$ & $82 \mathrm{mg}$ & 14,470 & 1.34 \\
\hline P3 & $72 \mathrm{~h}$ & $157 \mathrm{mg}$ & 15,892 & 1.26 \\
\hline
\end{tabular}




\section{Recycling Preparative HPLC Chromatograms for Polymers}
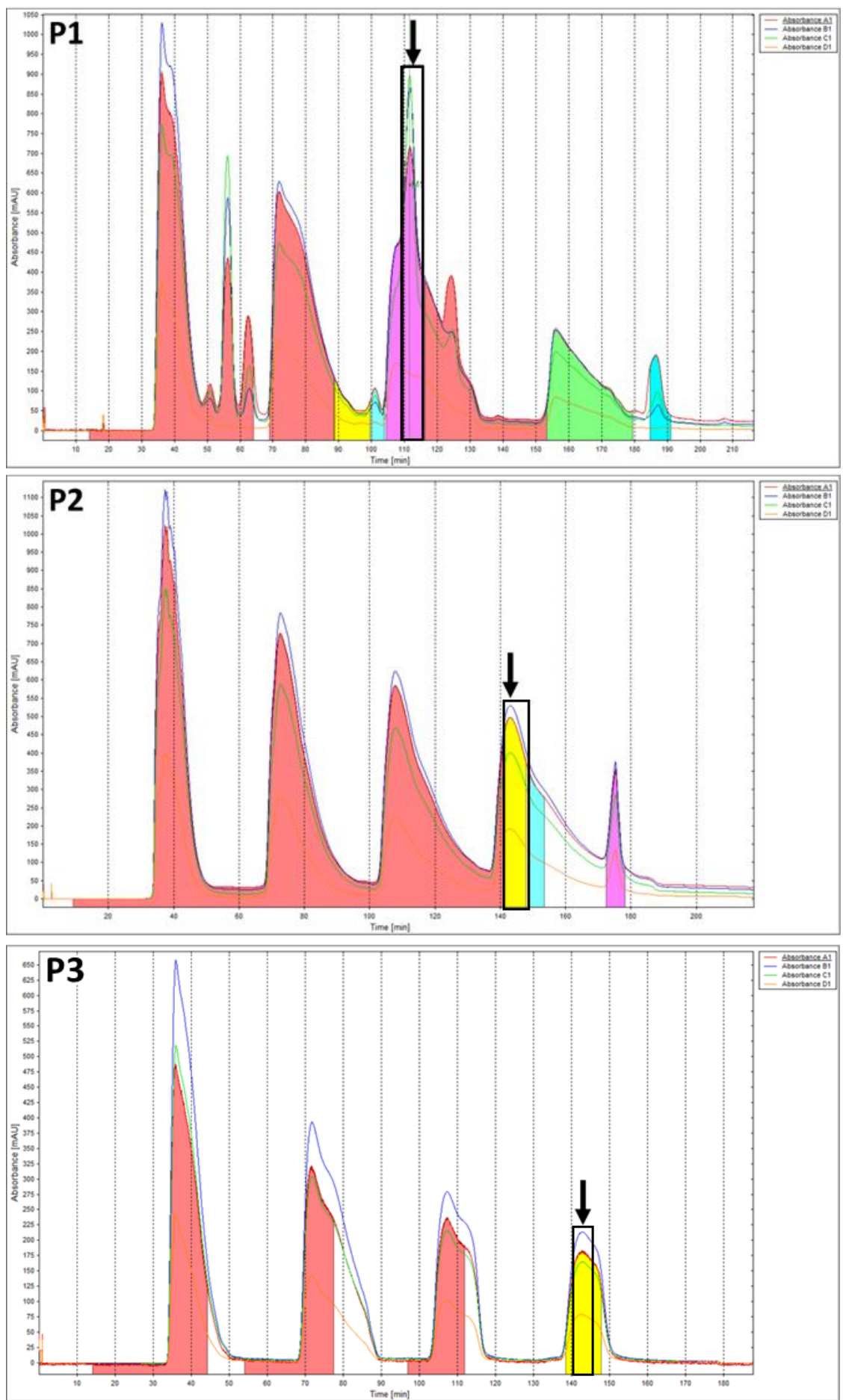

Figure S1. Preparative HPLC chromatograms of P1, P2, and P3. 
3. PHYSICAL CHARACTERIZATIONS

Gel Permeation Chromatography (GPC)

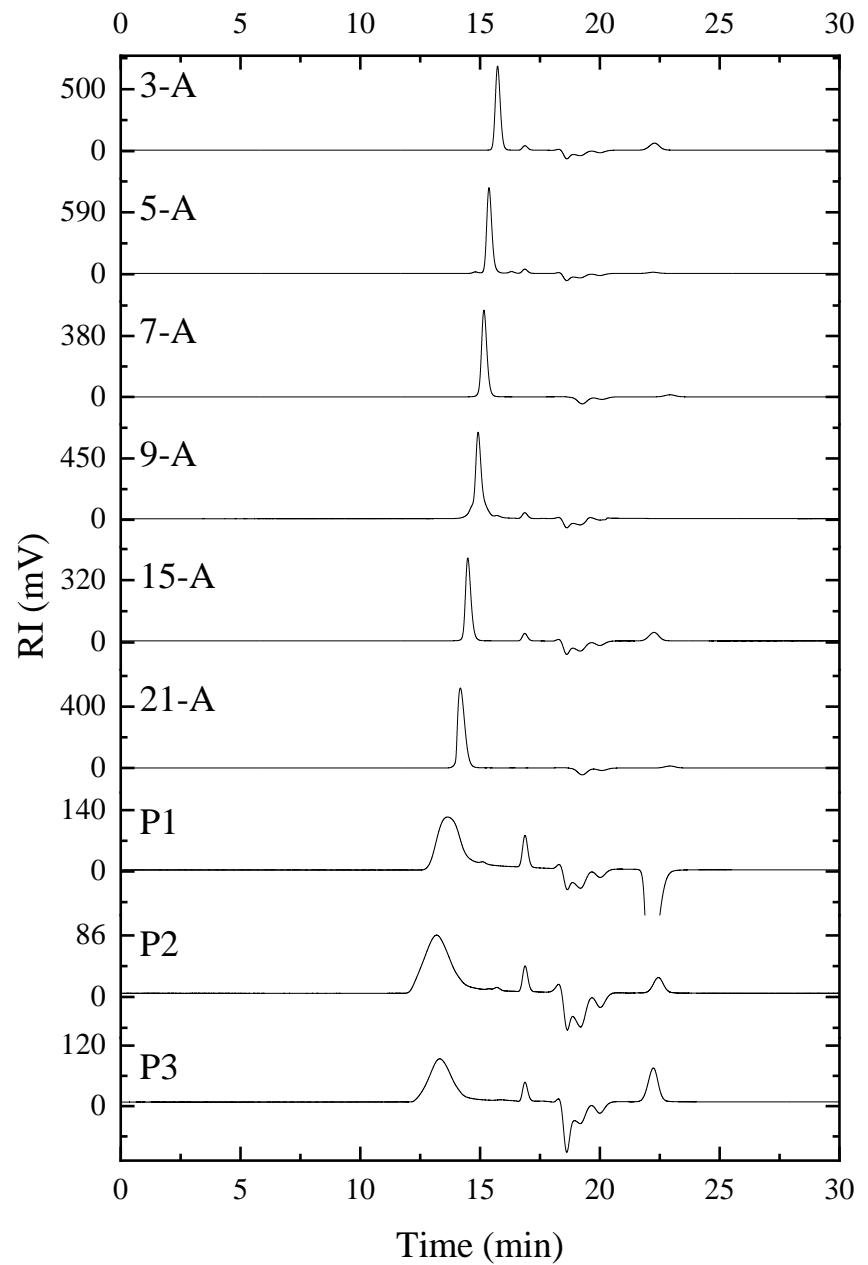

Figure S2. Raw GPC chromatogram for $\boldsymbol{n P B}$ series. 
Oligomer $\mathbf{n}=3$

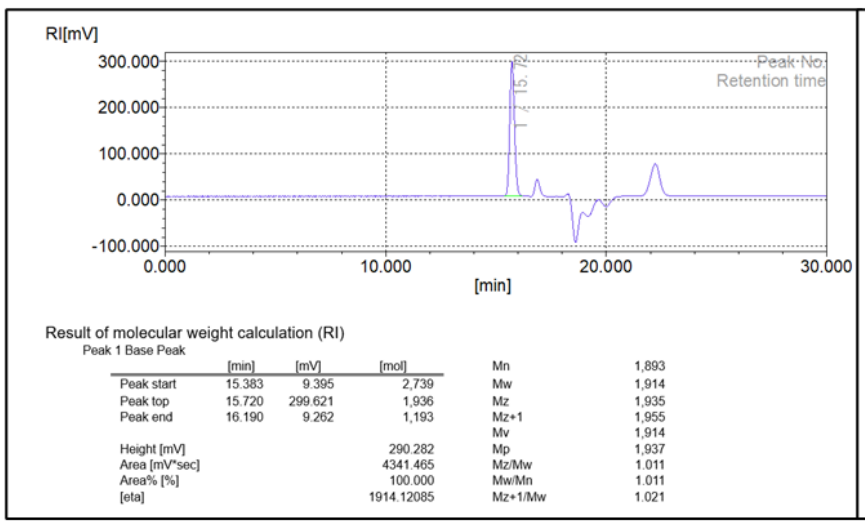

\section{Oligomer $\mathbf{n}=7$}

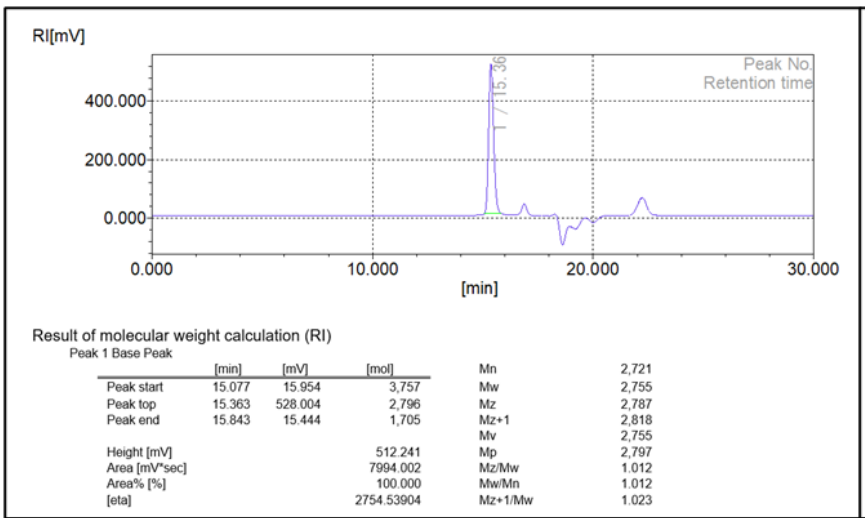

\section{Oligomer $\mathbf{n}=15$}

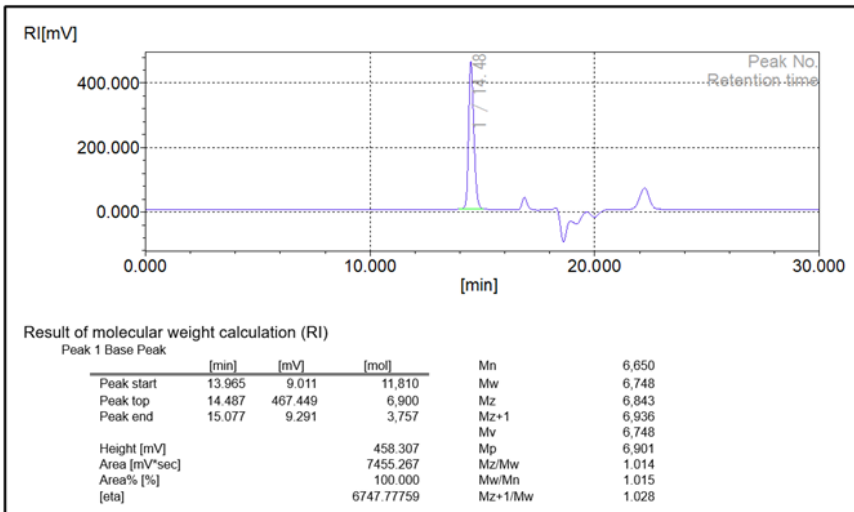

Oligomer $\mathbf{n}=\mathbf{5}$

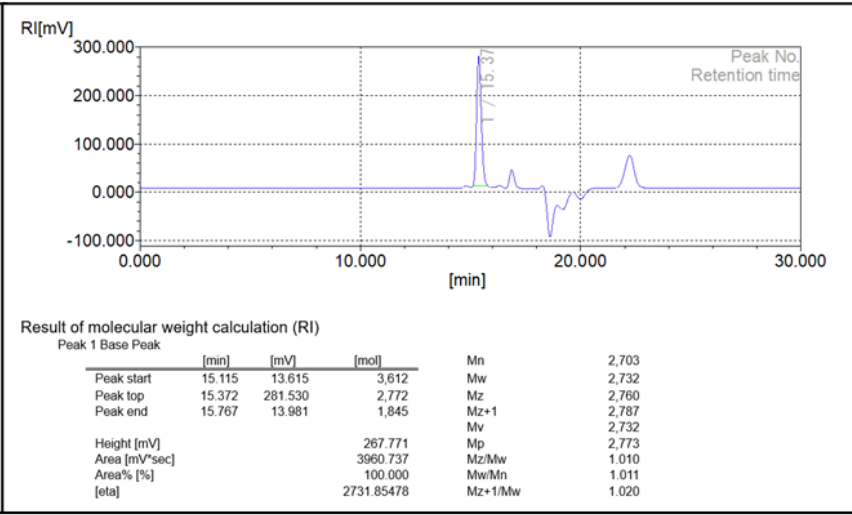

Oligomer $\mathbf{n}=9$

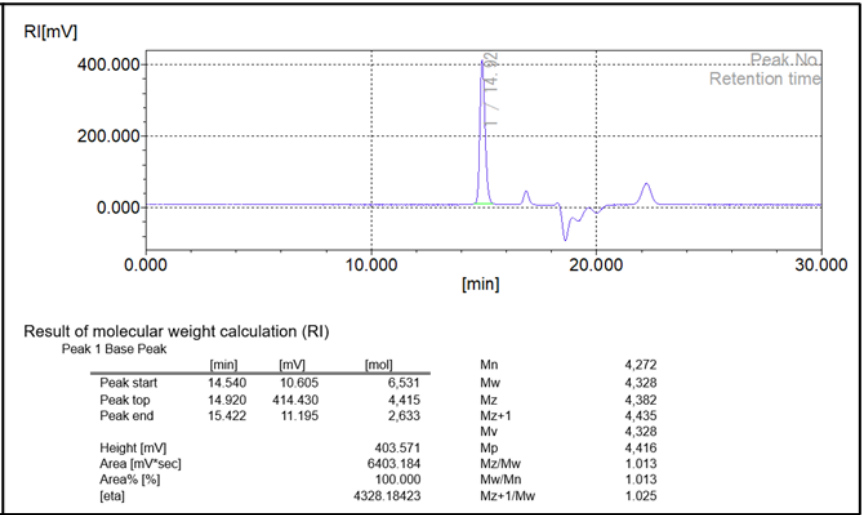

\section{Oligomer $\mathbf{n}=\mathbf{2 1}$}

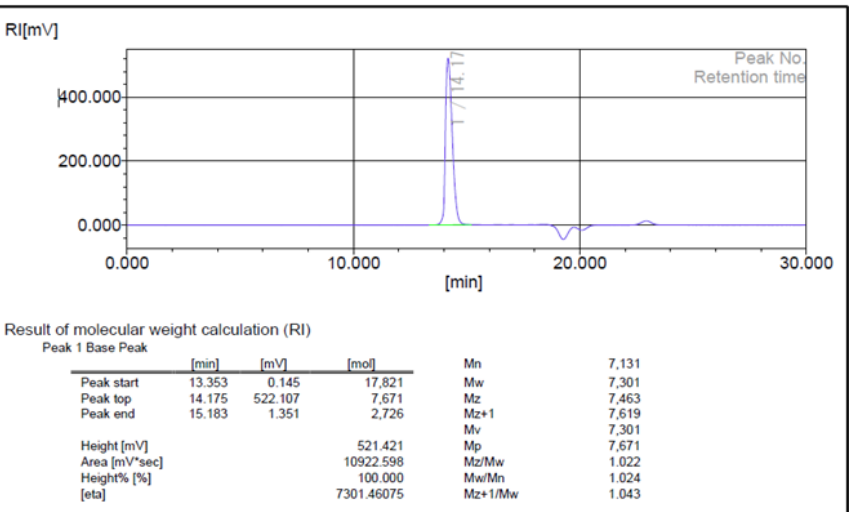

Figure S3. Raw GPC chromatograms for $\boldsymbol{n P B}$ oligomers. 
P1

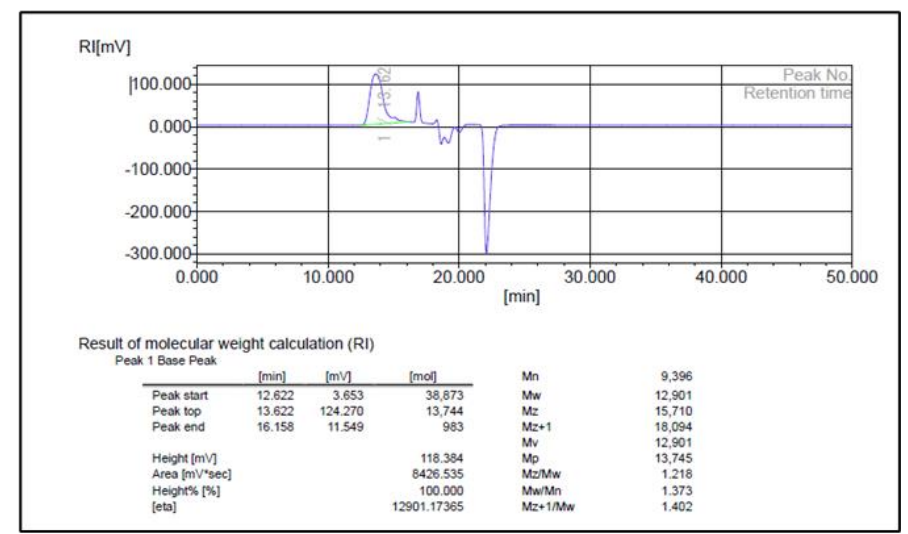

P2

RIImV]

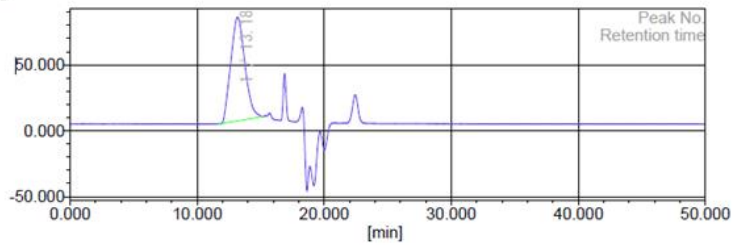

Result of molecular weight calculation (RI)

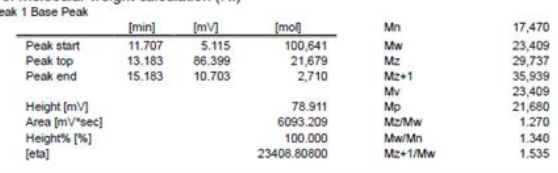

P3

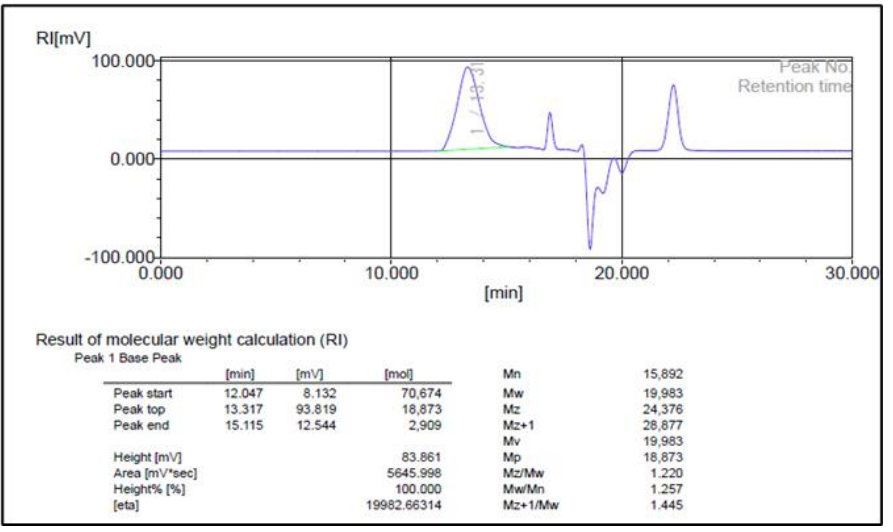

Figure S3. (continued) Raw GPC chromatograms for $\boldsymbol{n P B}$ polymers. 


\section{Electrochemistry}

\section{Electrochemical window stability}

0.2 $\mathrm{M} \mathrm{TBAPF}_{6}$ - Dichloromethane electrolyte system at a Pt wire ultramicroelectrode vs. $\mathrm{Ag} / \mathrm{AgCl}$ under nitrogen. Degradation of electrolyte begins to occur at $2.2 \mathrm{~V}$. All voltammetry was conducted under $2.1 \mathrm{~V}$.

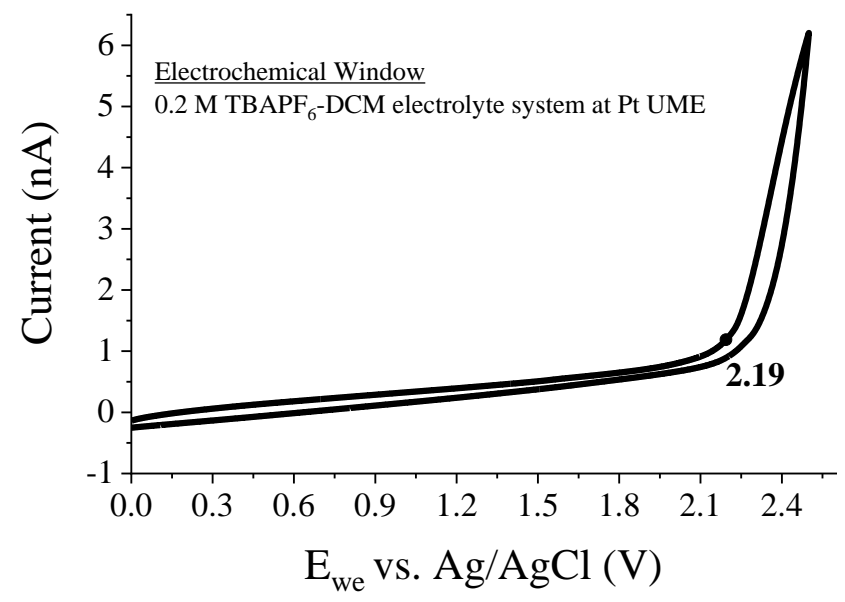

Figure S4. Electrochemical window stability of $0.2 \mathrm{M} \mathrm{TBAPF} 6-\mathrm{CH}_{2} \mathrm{Cl}_{2}$ at $25 \mathrm{~mm} \mathrm{Pt}$ ultramicroelectrode vs. $\mathrm{Ag} / \mathrm{AgCl}$ from 0 to $2.2 \mathrm{~V}$ under nitrogen atmosphere.

\section{Voltammetry}

Solution CV and DPV of the oligomer series dissolved at $\sim 1 \mathrm{mM}$ in $0.2 \mathrm{M} \mathrm{TBAPF}_{6} / \mathrm{CH}_{2} \mathrm{Cl}_{2}$ was conducted under a nitrogen atmosphere on a ultramicroelectrode $(25 \mathrm{~mm})$ platinum button electrode and a platinum wire counter electrode. $\mathrm{An} \mathrm{Ag} / \mathrm{AgCl}$ wire was used as the pseudoreference electrode which was calibrated against a ferrocene/ ferrocenium as an internal standard $\left(\mathrm{E}_{1 / 2}=0.51 \mathrm{~V}\right)$. For $\mathrm{CV}$, the voltage was scanned from 0 to $2.1 \mathrm{~V} \mathrm{vs.} \mathrm{Ag} / \mathrm{AgCl}$, at a $10 \mathrm{mV} / \mathrm{s}$ scan rate. The second cycle is reported in the main text figure. For DPV, the voltage was scanned from 0 to $2.1 \mathrm{~V}$ vs. $\mathrm{Ag} / \mathrm{AgCl}$ in $0.004 \mathrm{~V}$ increments, at $0.05 \mathrm{~V}$ amplitude, with a $0.05 \mathrm{sec}$ pulse width, a $0.0167 \mathrm{sec}$ sample width, and a $0.5 \mathrm{sec}$ pulse period. For NDPV, all DPV parameters were kept the same with the exception of a 0.2 sec pulse period. DPV deconvolution was achieved by calculating semi-derivatives of the current-voltage voltammograms via the CHI 660 potentiostat software. 

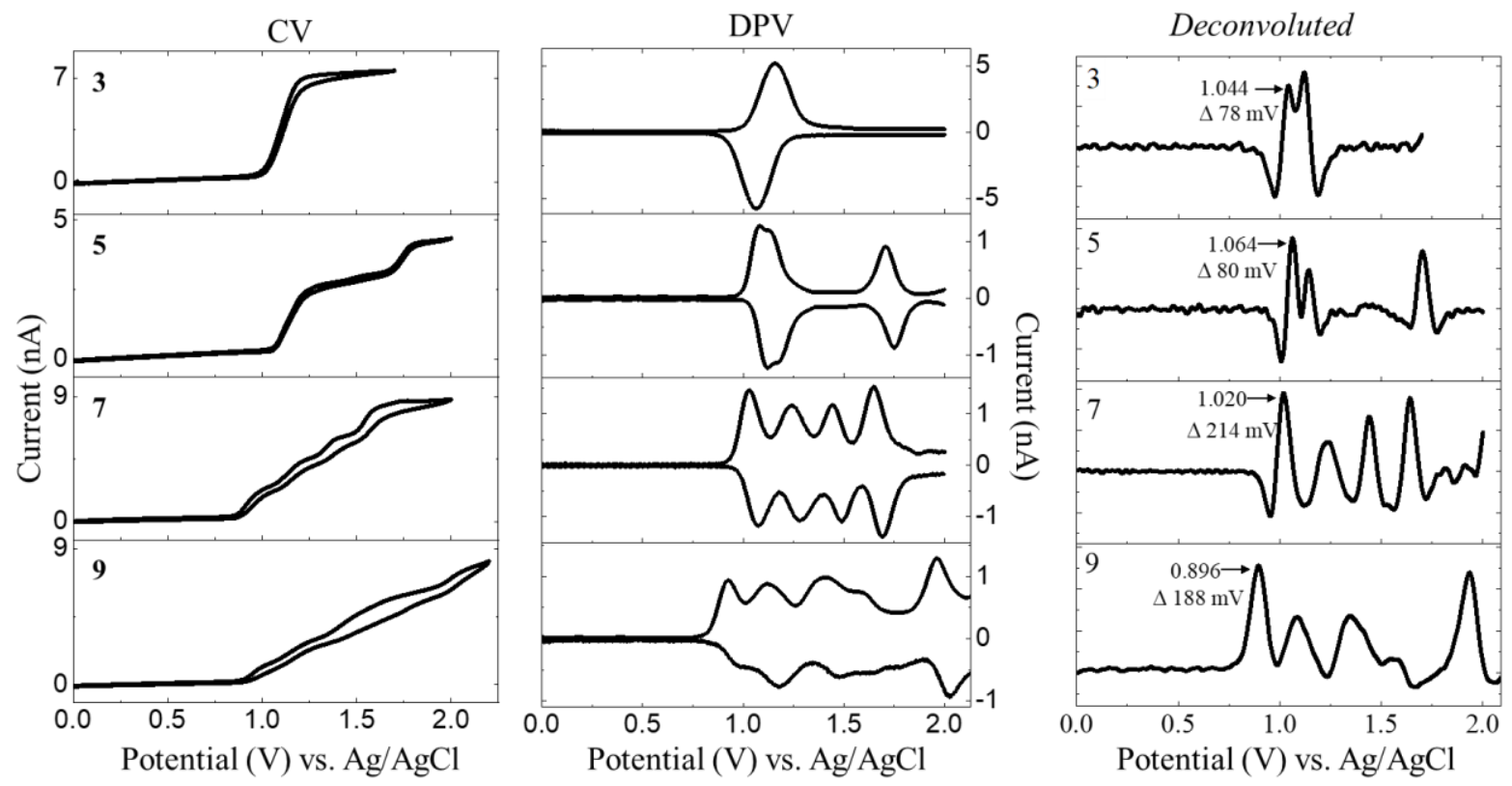

Figure S5. Raw and deconvoluted CV and DPV voltammograms for n=3-9.

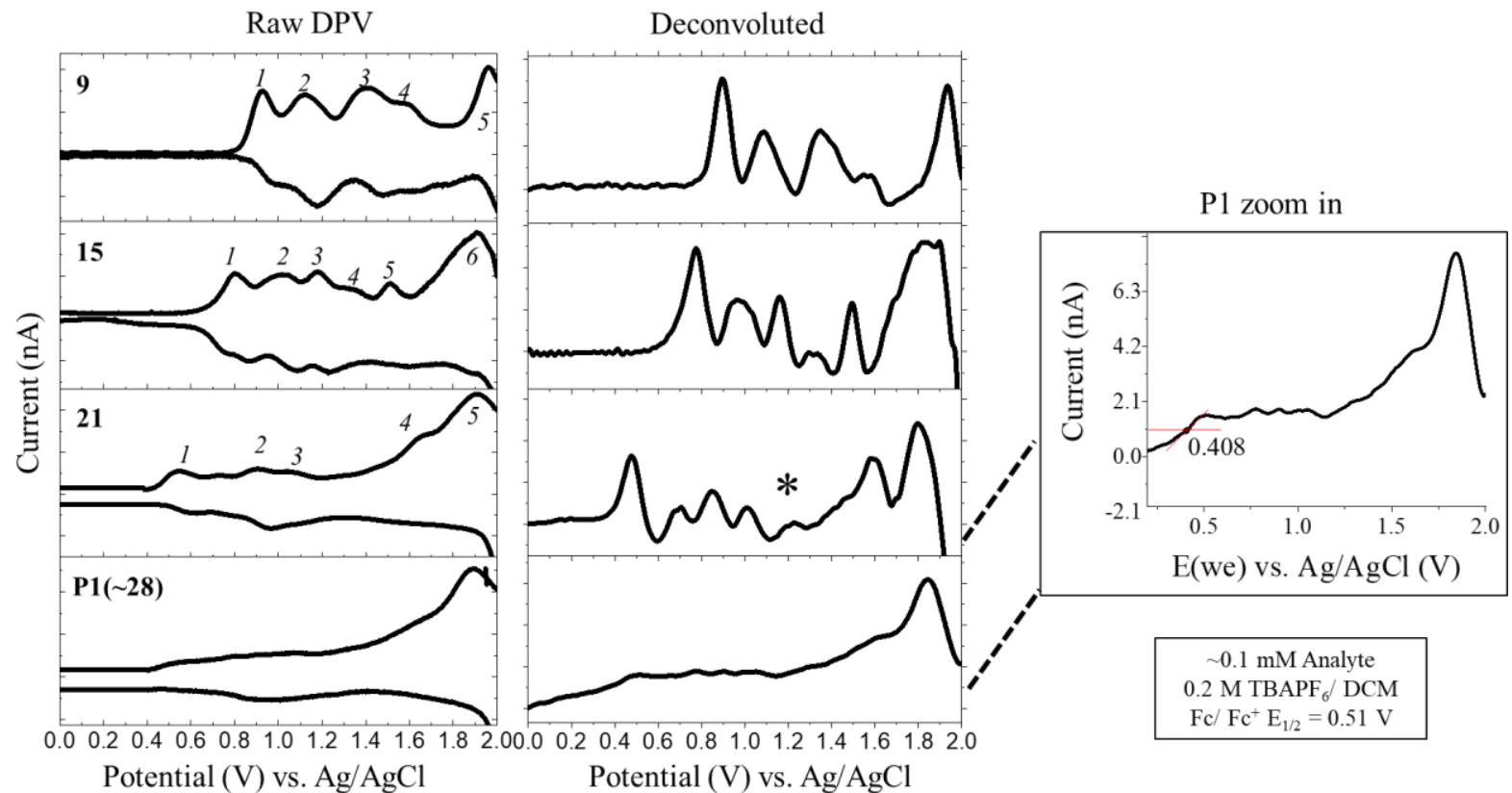

Figure S6. Raw and deconvoluted CV and DPV voltammograms for $\mathrm{n=9-21}$ and $\mathbf{P 1}$. 


\section{Spectroelectrochemistry}

Solution spectroelectrochemistry was done in a three electrode honeycomb electrochemical cell supplied by Pine Research. A $12.5 \times 12.5 \times 45.0 \mathrm{~mm}^{3}$ quartz cuvette with a $1.7 \mathrm{~mm}$ path length was used as the cell, equipped with a polished platinum honeycomb electrode (working and counter) and $\mathrm{Ag} / \mathrm{AgCl}$ pseudoreference electrode calibrated against a ferrocene/ferrocenium standard for which the $\mathrm{E}_{1 / 2}$ is taken to be $0.51 \mathrm{~V}$ vs. $\mathrm{Ag} / \mathrm{AgCl}$. A homemade teflon cap was specially designed to keep the cell sealed from solvent evaporation and air contamination. In a nitrogen filled glove box, $4 \times 10^{-4} \mathrm{M}$ solution of $\mathbf{P B P}$ was prepared with $0.2 \mathrm{M} \mathrm{TBAPF}_{6}$ in anhydrous DCM and transferred and sealed in the electrochemical quartz cell and sealed with teflon tape. During electrochemical oxidation, the voltage is increased from 0 to $1.2 \mathrm{~V}$ in $50 \mathrm{mV}$ increments by linear scan voltammetry (LSV) at a $25 \mathrm{mV} / \mathrm{s}$ scan rate.
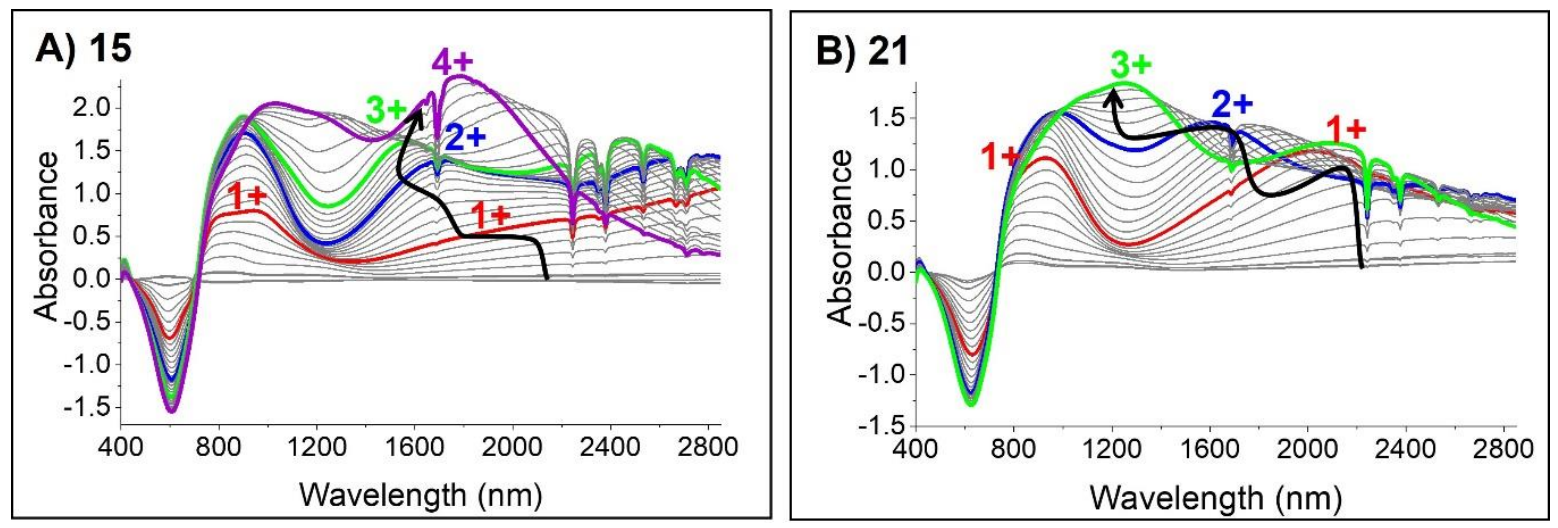

Figure S7: Spectroelectrochemistry of A) 15 and B) 21 in $0.2 \mathrm{M} \mathrm{TBAPF}_{6} / \mathrm{CH}_{2} \mathrm{Cl}_{2}$. This experiment was conducted in an air-tight optically transparent thin-layer quartz cuvette equipped with a $\mathrm{Pt}$ honeycomb electrode under nitrogen.

\begin{tabular}{|c|c|c|c|c|}
\hline $\mathbf{n}$ & \multicolumn{2}{|c|}{$\mathbf{+ 3}$} & \multicolumn{2}{c|}{$\begin{array}{c}\mathbf{4} \\
(\mathbf{e V}, \mathbf{n m})\end{array}$} \\
\hline $\mathbf{3}$ & -- & -- & -- & -- \\
\hline $\mathbf{5}$ & $\mathrm{NR}$ & $\mathrm{NR}$ & -- & -- \\
\hline $\mathbf{7}$ & 0.94 & 1314 & $\mathrm{NR}$ & $\mathrm{NR}$ \\
\hline $\mathbf{9}$ & $0.76 / 0.90$ & $1636 / 1375$ & 0.90 & 1375 \\
\hline $\mathbf{1 5}$ & $1.32 / 0.85 / 0.62-0.44^{(\mathrm{b})}$ & $940 / 1465 / 2000-2800(\mathrm{~b})$ & $1.23 / 0.70$ & $1010 / 1778$ \\
\hline $\mathbf{2 1}$ & $0.98 / 0.58$ & $1260 / 2140$ & $\mathrm{NR}$ & $\mathrm{NR}$ \\
\hline P1 & NR & $\mathrm{NR}$ & $\mathrm{NR}$ & $\mathrm{NR}$ \\
\hline (b)= broad & & & \\
NR = not resolvable & & & \\
\hline
\end{tabular}

Table S1. Photophysical properties $\left(\lambda_{\max }\right)$ of the +3 and +4 oxidation states of the $n \mathbf{P B}$ series by spectroelectrochemistry in solution. 


\section{Atomic Force Microscopy (AFM)}
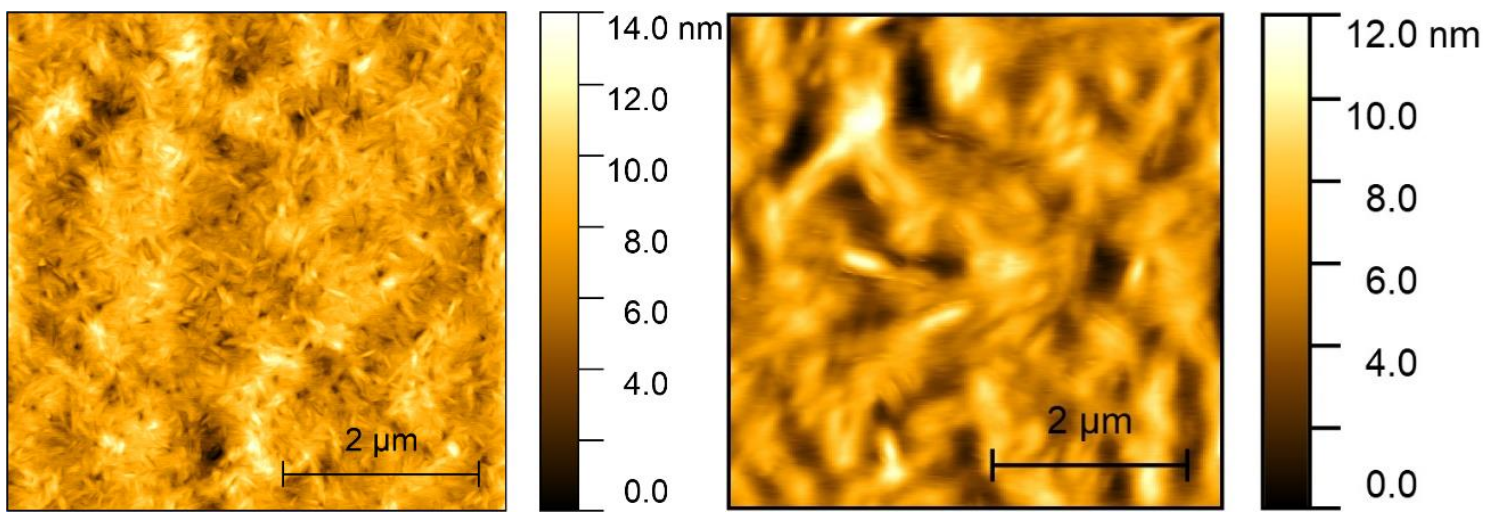

Figure S8. AFM image showing crystallite formation of solution cast $n=5$ from chloroform as a thin film.

\section{Small Angle Neutron Scattering (SANS)}

Dilute solutions of oligomers and polymers were prepared $(5 \mathrm{mg} / \mathrm{mL})$ in chlorobenzene-d5. Chlorobenzene-d5 (D $>$ 99\%) and was purchased from Cambridge Isotopes (Tewksbury, MA) and used as received. SANS measurements were carried out using the Extended Q-Range Small-Angle Neutron Scattering Diffractometer (EQ-SANS) at the Spallation Neutron Source (SNS), ORNL. The scattering wavevector q ranged from 0.003 to $0.8 \AA-1$, by using two different configurations ( $4 \mathrm{~m} 12 \AA$ and $2.5 \mathrm{~m} 2.5 \AA$ ). The samples were contained in Hellma quartz cells with a 2 $\mathrm{mm}$ path length. Measurements were performed at $75{ }^{\circ} \mathrm{C}$. The absolute intensity was obtained through data reduction and correction. Reduced data was later fitted to a flexible cylinder model in SasView.24.

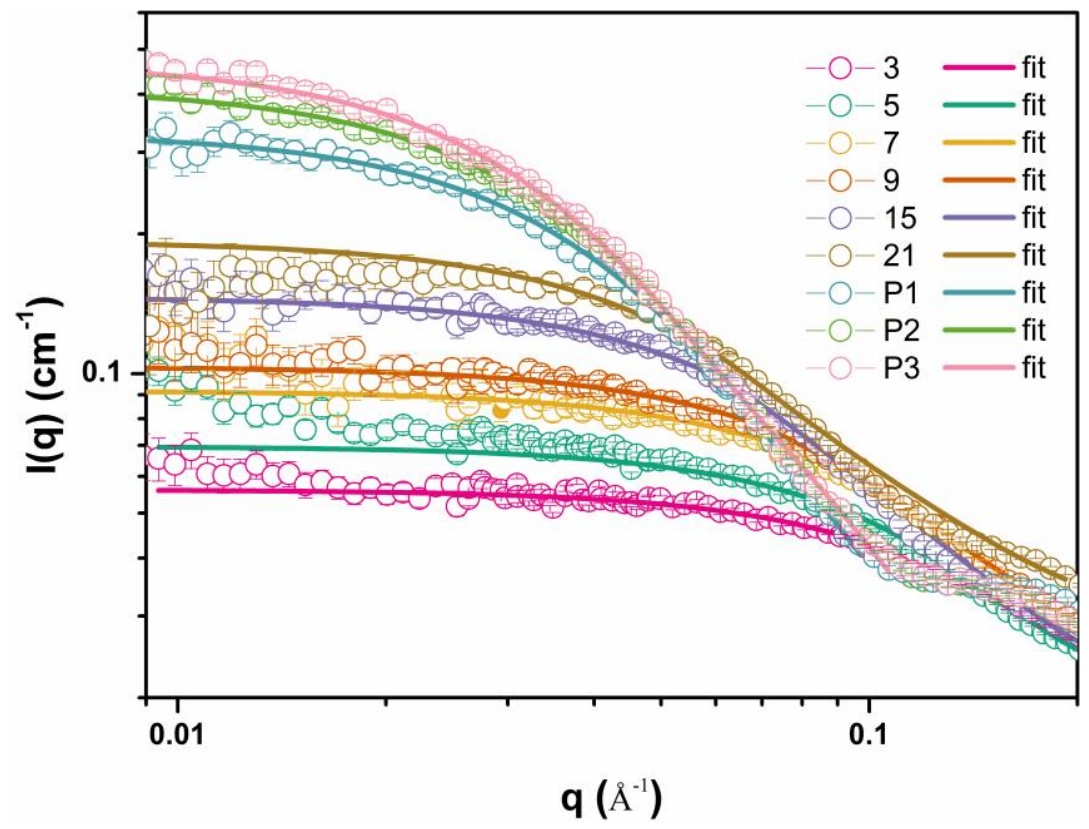

Figure S9. Comparison of scattering profiles for oligomers and polymers with different molecular weights at a concentration of $5 \mathrm{mg} / \mathrm{mL}$. 


\begin{tabular}{|c|c|c|c|}
\hline $\mathbf{n}$ & $\begin{array}{c}\text { Persistence } \\
\text { Length } \\
(\mathbf{n m})\end{array}$ & $\begin{array}{c}\text { Contour } \\
\text { Length } \\
(\mathbf{n m})\end{array}$ & $\begin{array}{c}\text { Radius } \\
(\mathbf{n m})\end{array}$ \\
\hline $\mathbf{3}$ & $1.28 \pm 0.2$ & $2.10 \pm 0.1$ & $1.24 \pm 0.02$ \\
\hline $\mathbf{5}$ & $1.77 \pm 0.4$ & $3.19 \pm 0.1$ & $1.24 \pm 0.02$ \\
\hline $\mathbf{7}$ & $2.30 \pm 0.4$ & $4.04 \pm 0.1$ & $1.24 \pm 0.02$ \\
\hline $\mathbf{9}$ & $2.37 \pm 0.2$ & $4.97 \pm 0.1$ & $1.24 \pm 0.02$ \\
\hline $\mathbf{1 5}$ & $2.56 \pm 0.1$ & $7.83 \pm 0.2$ & $1.24 \pm 0.02$ \\
\hline $\mathbf{2 1}$ & $2.61 \pm 0.2$ & $11.37 \pm 0.2$ & $1.24 \pm 0.02$ \\
\hline P1 ( 28) & $2.70 \pm 0.2$ & $20.04 \pm 0.1$ & $1.24 \pm 0.02$ \\
\hline P2( 40) & $2.89 \pm 0.1$ & $23.08 \pm 0.2$ & $1.24 \pm 0.02$ \\
\hline P3( 48) & $3.12 \pm 0.2$ & $24.92 \pm 0.1$ & $1.24 \pm 0.02$ \\
\hline
\end{tabular}

Table S2. Parameters obtained from fits to SANS data with the flexible cylinder model. And contour length dispersities are 1. 


\section{Theoretical Calculations}

\begin{tabular}{|c|c|c|c|c|c|}
\hline & $\mathbf{5}$ & $\mathbf{7}$ & $\mathbf{9}$ & $\mathbf{1 5}$ & $\mathbf{1 9}$ \\
\hline $\mathbf{I P}$ & 5.00 & 4.88 & 4.84 & 4.82 & 4.82 \\
\hline $\mathbf{E}_{\mathbf{H L}}$ & 4.64 & 4.34 & 4.12 & 3.88 & 3.94 \\
\hline $\boldsymbol{\omega}$ & 0.1112 & 0.1067 & 0.0974 & 0.0931 & 0.0985 \\
\hline
\end{tabular}

Table S3.. Adiabatic ionization potential (IP), HOMO-LUMO gap $\left(\mathrm{E}_{\mathrm{HL}}\right)$, and optimally tuned long-range correction parameter for $\mathrm{n}=5-19$ as determined at the OT- $\omega \mathrm{B} 97 \mathrm{X}-\mathrm{D} / 6-31 \mathrm{G}(\mathrm{d}, \mathrm{p})$ level of theory.
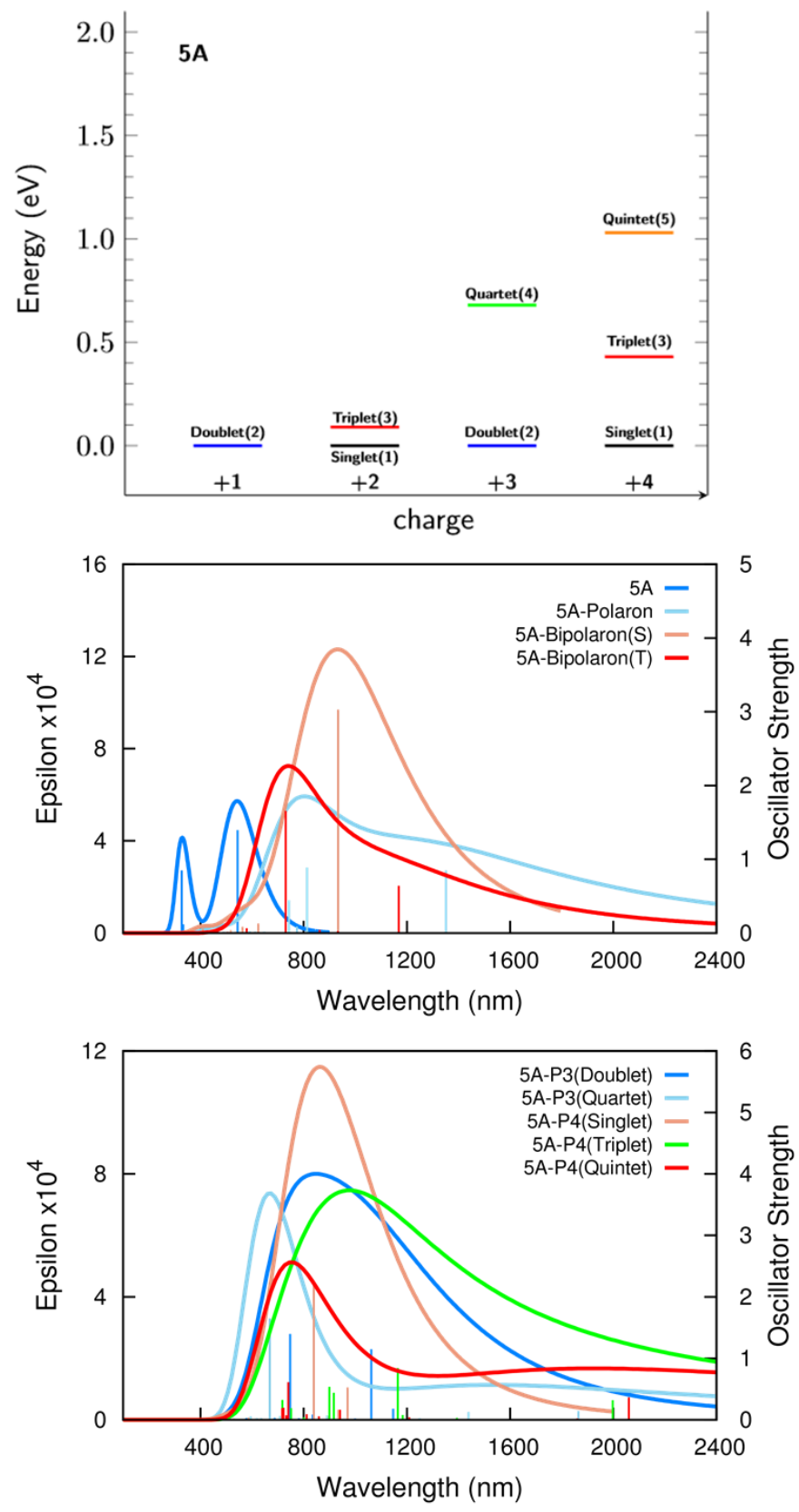

Figure S10. (top) DFT-determined energies of select spin states as a function of oxidation, and (middle, bottom) TDDFT simulated optical absorption profiles [via Gaussian convolution using a FWHM=0.3 eV] for select spin states for $\mathrm{n}=5$ as determined at the at the OT- $\omega$ B97X-D/6-31G(d,p) level of theory. 

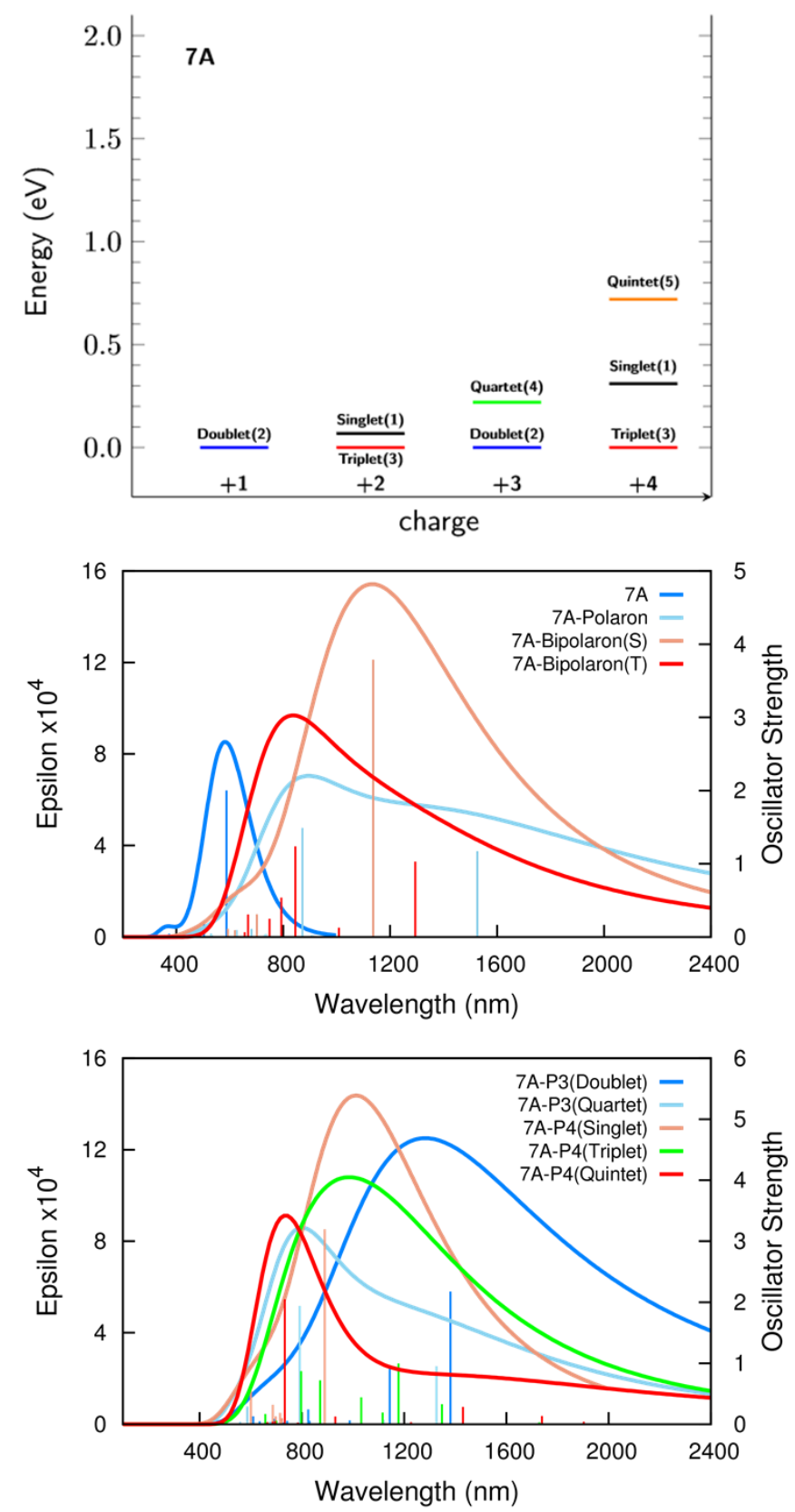

Figure S11. (top) DFT-determined energies of select spin states as a function of oxidation, and (middle, bottom) TDDFT simulated optical absorption profiles [via Gaussian convolution using a FWHM=0.3 eV] for select spin states for $\mathrm{n}=7$ as determined at the at the OT- $\omega$ B97X-D/6-31G(d,p) level of theory. 

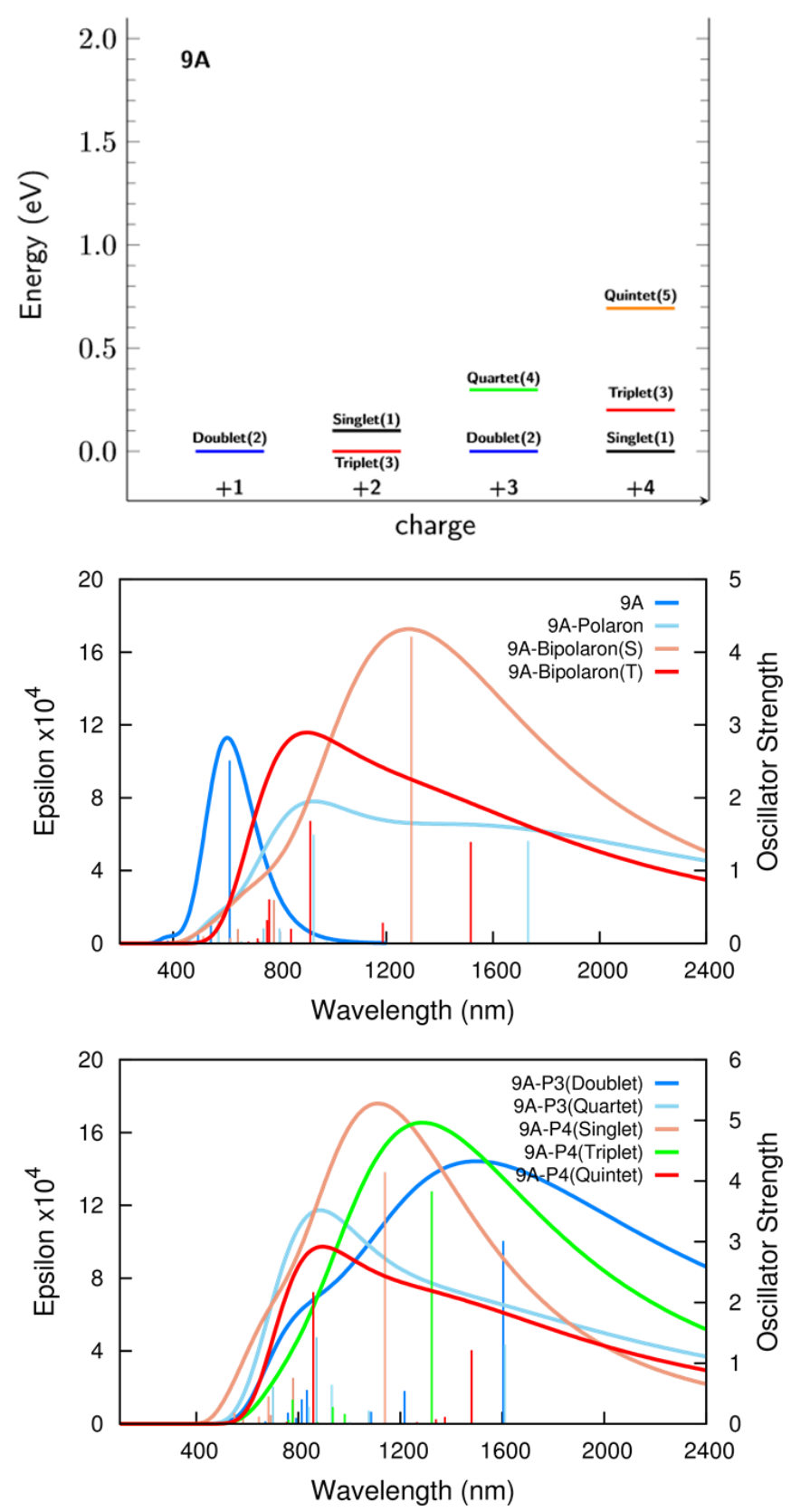

Figure S12. (top) DFT-determined energies of select spin states as a function of oxidation, and (middle, bottom) TDDFT simulated optical absorption profiles [via Gaussian convolution using a FWHM=0.3 eV] for select spin states for $\mathrm{n}=9$ as determined at the at the OT- $\omega$ B97X-D/6-31G(d,p) level of theory. 

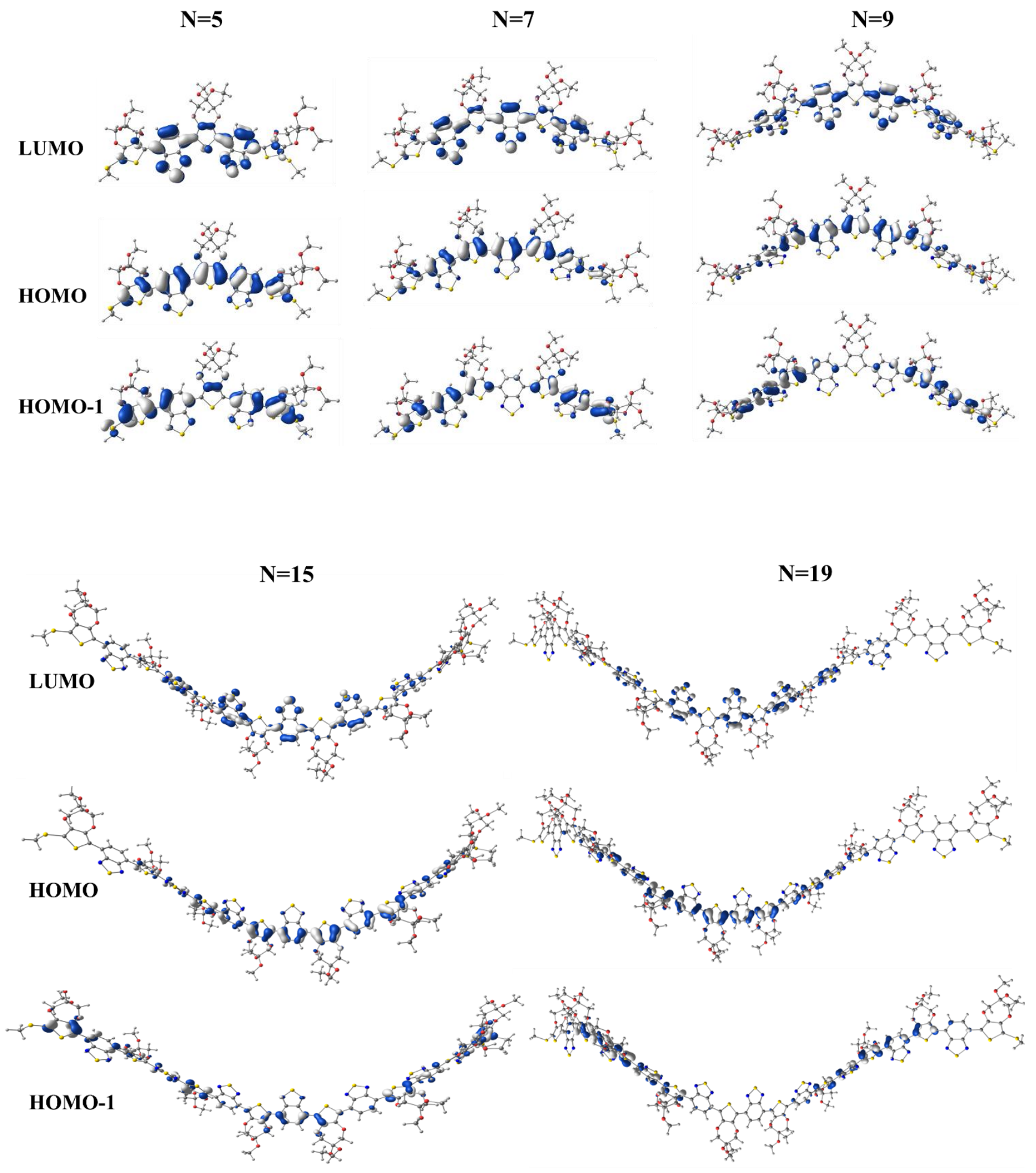

Figure S13: LUMO, HOMO, and HOMO-1 orbitals of $n=5,7,9,15$, and 19. 
5
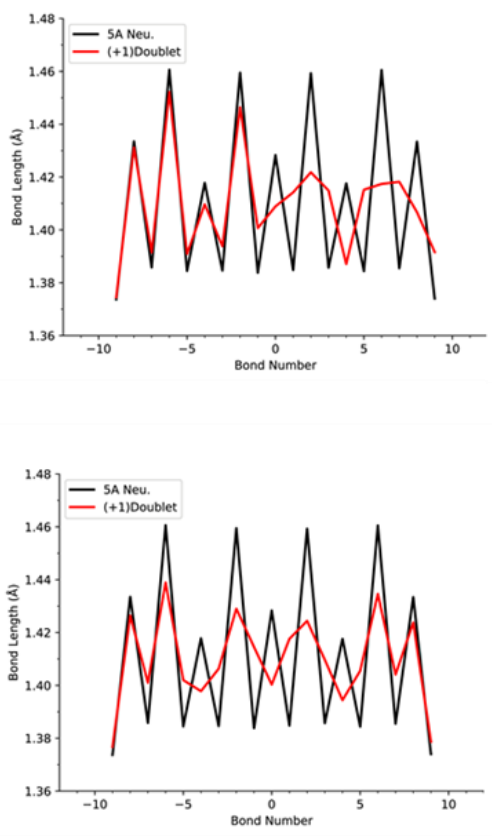

15
7
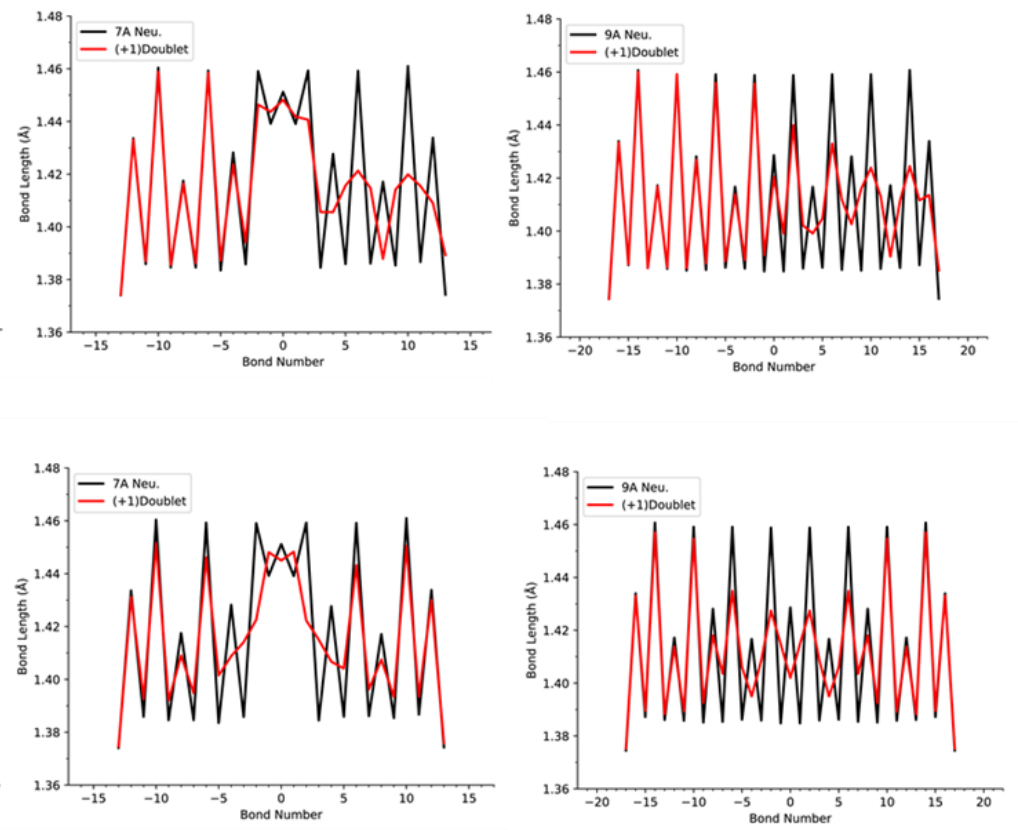

9

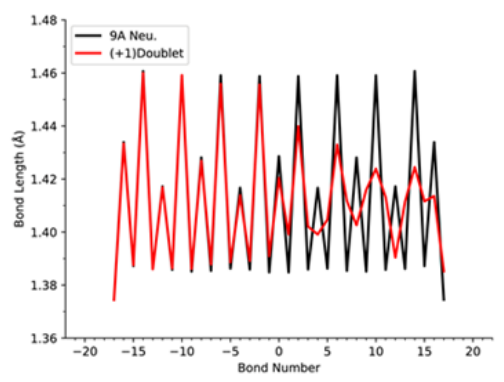

19
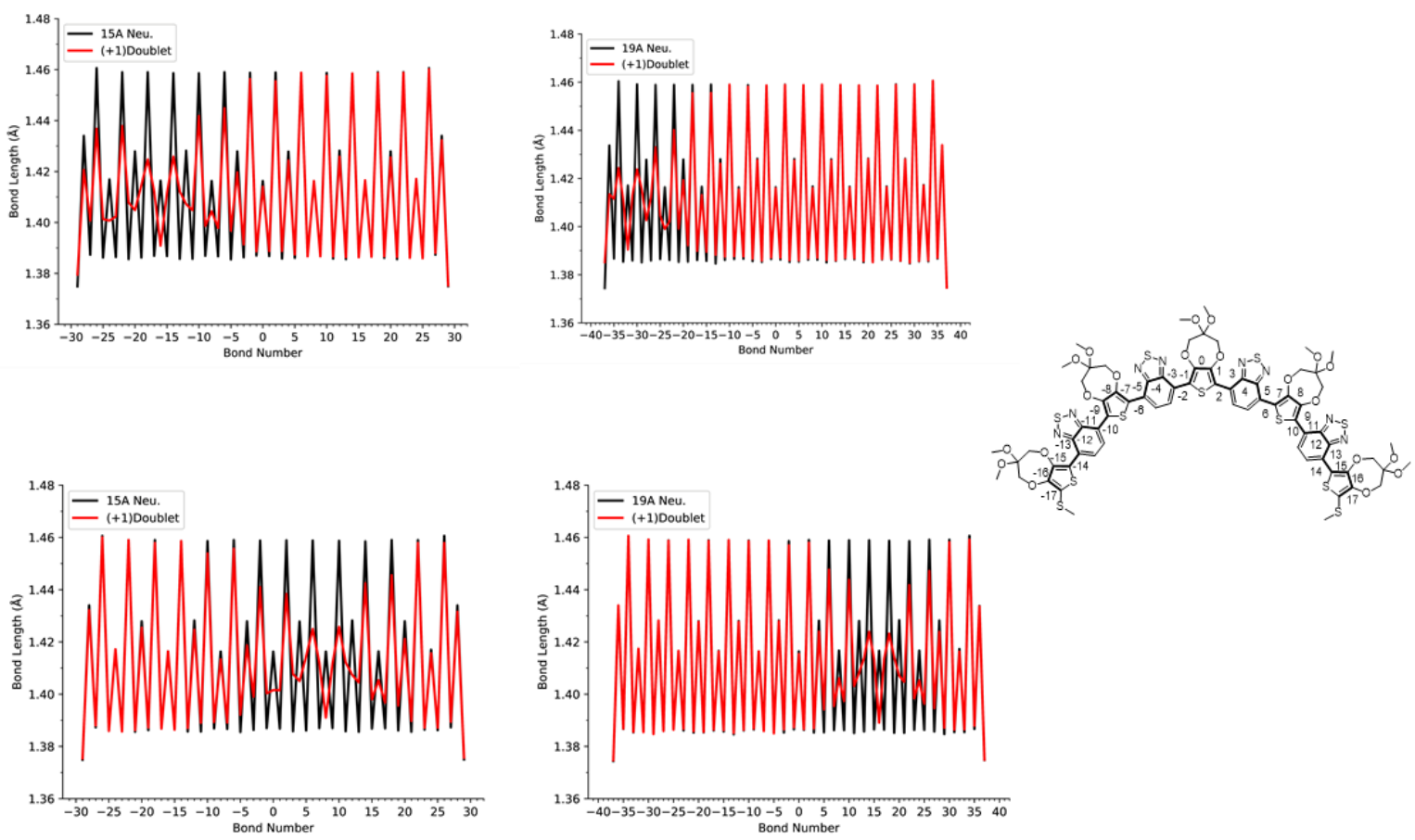

Figure S14. Bond lengths across the $\mathrm{C}-\mathrm{C} \pi$-conjugated backbones of $\mathrm{n}=5,7,9,15$, and 19 for the neutral (black) and radical-cation (red) states as determined as determined at the at the OT- $\omega$ B97X-D/6-31G(d,p) level of theory. Note that for each oligomer, the top image is for the symmetry-broken solution (obtained by twisting the methylthiol group as described in the main text), while the bottom is for the symmetric geometric orientation. The bond numbering scheme for $\mathrm{n}=9$ is provided as an example. 


\section{Nuclear Magnetic Resonance (NMR) Spectra}

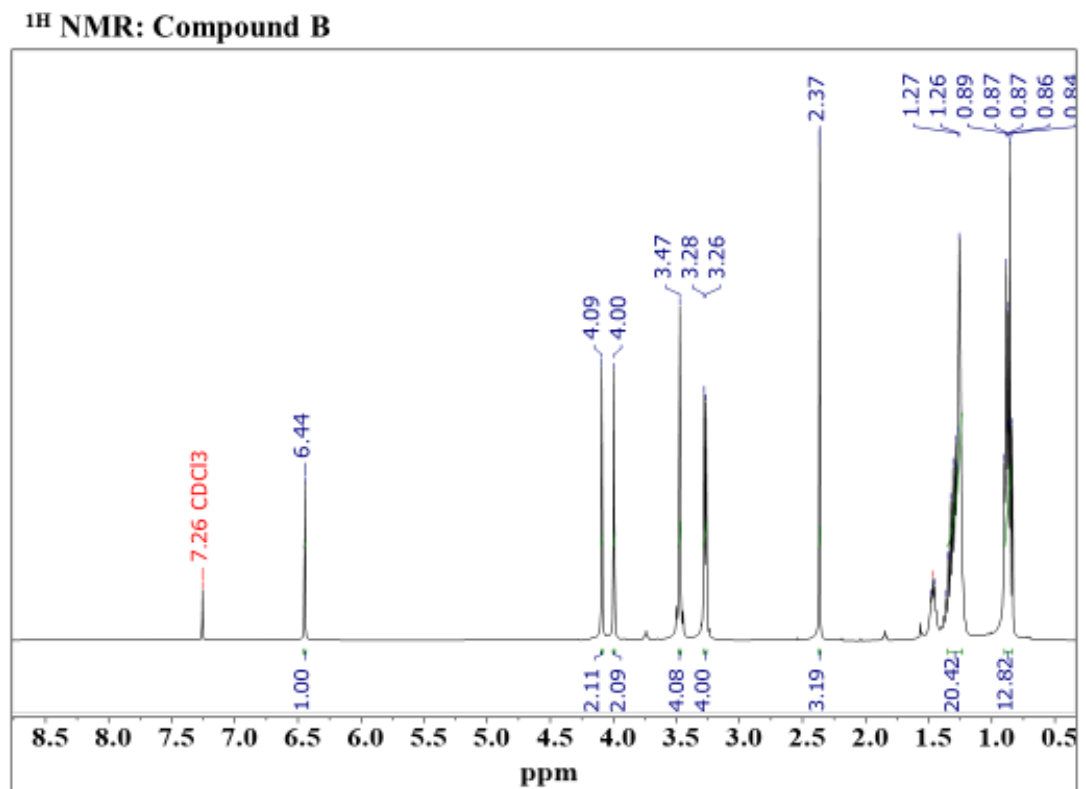

3,3-bis(((2-ethylhexyl)oxy)methyl)-6-(methylthio)-3,4-dilyydro-2H-thieno[3,4-b][1,4]dioxepine ( $\left.{ }^{\mathrm{H}} \mathrm{H} \mathrm{NMR}, \mathrm{CDCl}_{3}, 300 \mathrm{MHz}\right)$

${ }^{13}$ C NMR: Compound B

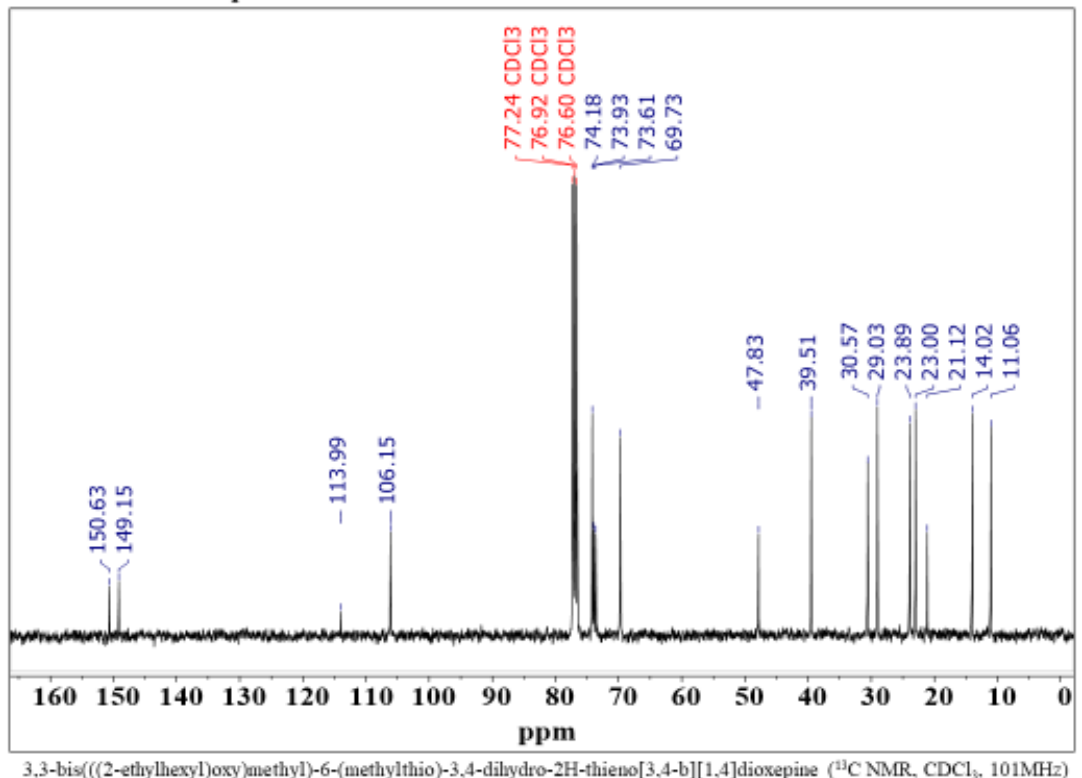


${ }^{1}$ H NMR: Compound D

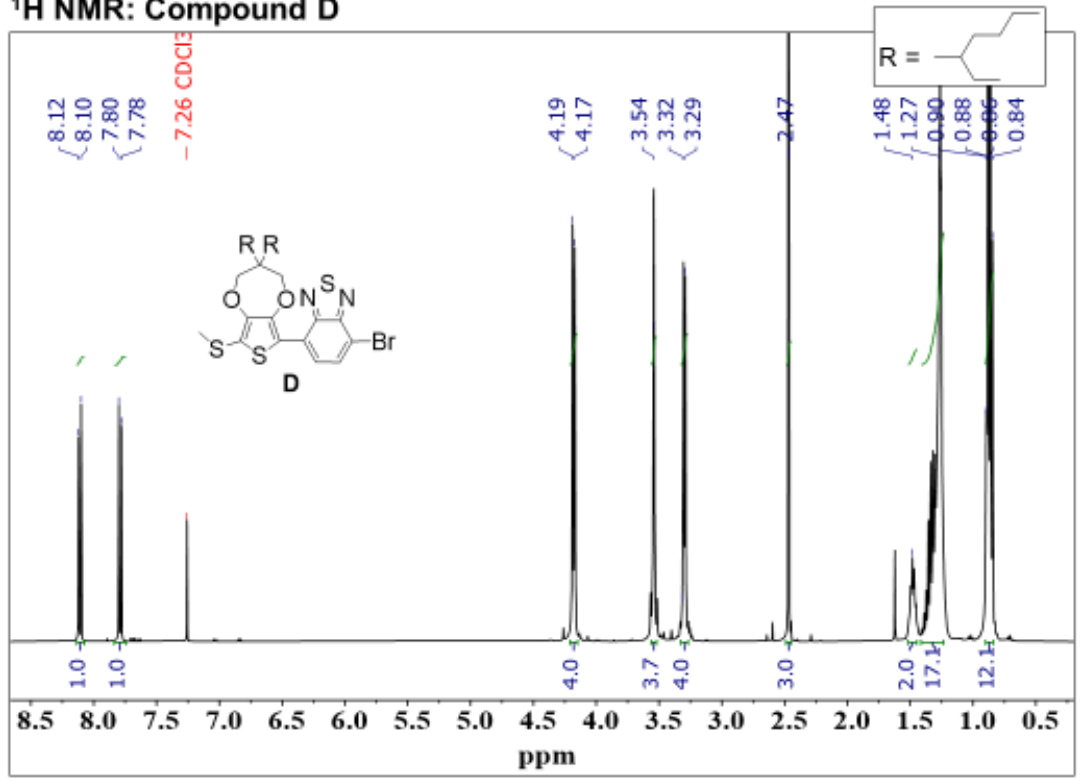

4-(3,3-bis(((2-ethylhexyl)oxy)methyl)-8-(methylthio)-3,4-dihydro-2H-thieno[3,4-b][1,4]dioxepin-6-yl)-7bromobenzo[c] $[1,2,5]$ thiadiazole $\left({ }^{1} \mathrm{H} \mathrm{NMR}, \mathrm{CDCl}_{5}, 400 \mathrm{MHz}\right)$

${ }^{13}$ C NMR: Compound D

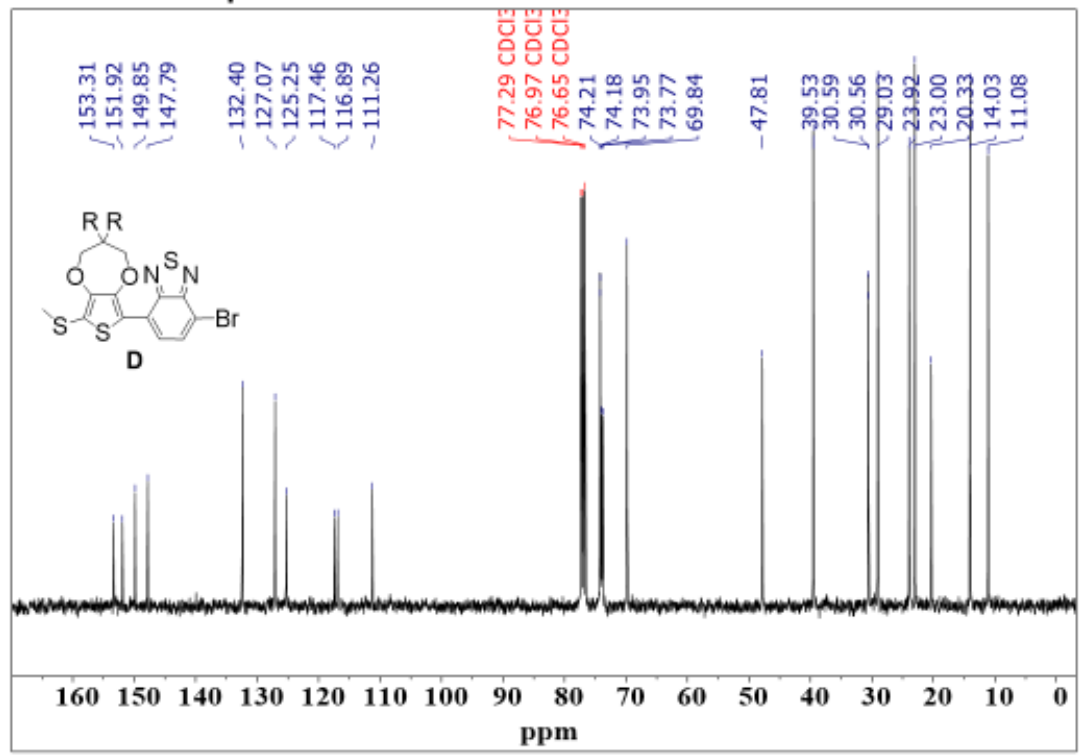

4-(3,3-bis(((2-ethylhexyl)oxy)methyl)-8-(methylthio)-3,4-dihydro-2H-thieno[3,4-b][1,4]dioxepin-6-yl)-7-

bromobenzo[c] $[1,2,5]$ thiadiazole $\left({ }^{13} \mathrm{C} \mathrm{NMR}, \mathrm{CDCl}_{5}, 101 \mathrm{MHz}\right)$ 
${ }^{1} \mathrm{H}$ NMR: Compound E

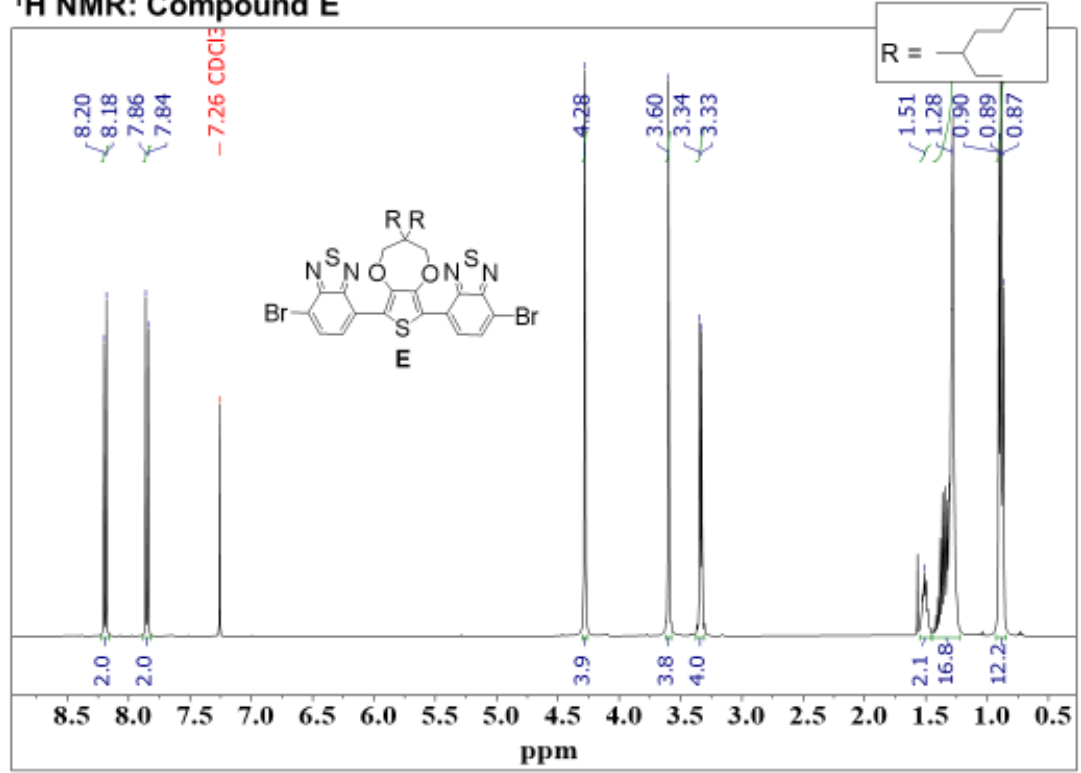

7,7'-(3,3-bis(((2-ethylhexyl)oxy)methyl)-3,4-dilyydro-2H-thieno[3,4-b][1,4]dioxepine-6,8-diyl)bis(4bromobenzo[c][1,2,5] thiadiazole) ( ${ }^{2} \mathrm{H} \mathrm{NMR}, \mathrm{CDCl}_{3}, 400 \mathrm{MHz}$ )

${ }^{13} \mathrm{C}$ NMR: Compound E

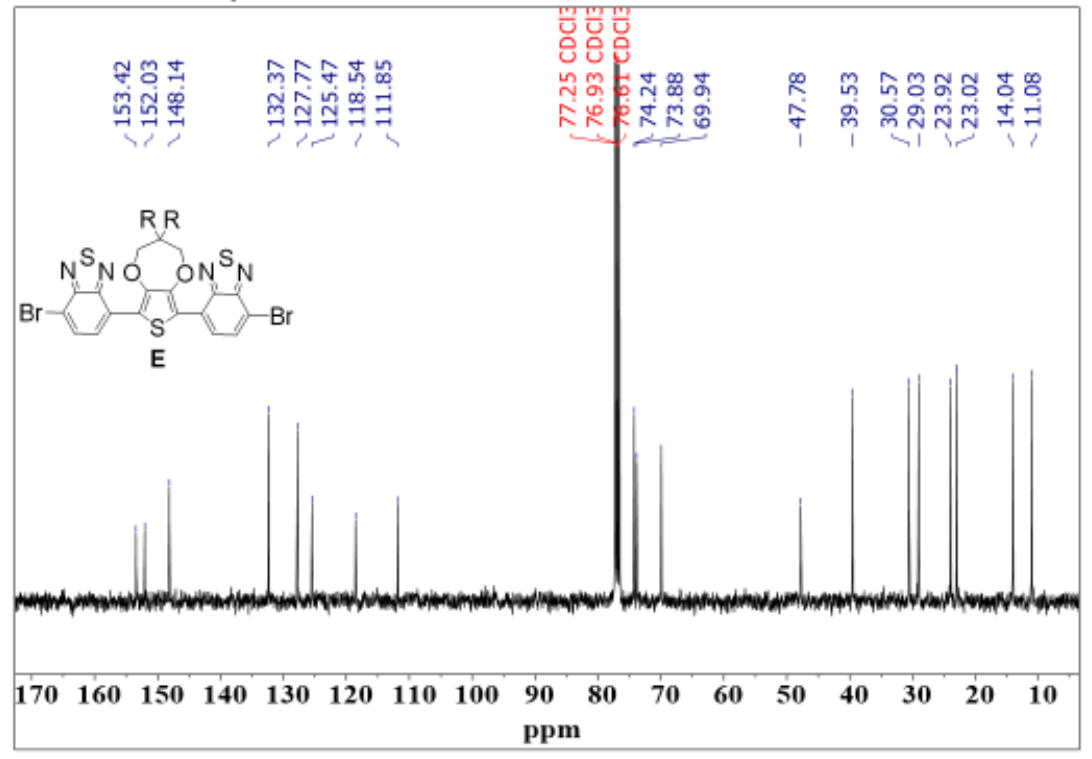

7,7'-(3,3-bis(((2-ethylhexyl)oxy)methyl)-3,4-dilhydro-2H-thieno[3,4-b][1,4] dioxepine-6,8-diyl)bis(4bromobenzo[c] $[1,2,5]$ thiadiazole) $\left({ }^{13} \mathrm{CNMR}, \mathrm{CDCl}_{3}, 101 \mathrm{MHz}\right)$ 
${ }^{1} \mathrm{H}$ NMR: Compound F

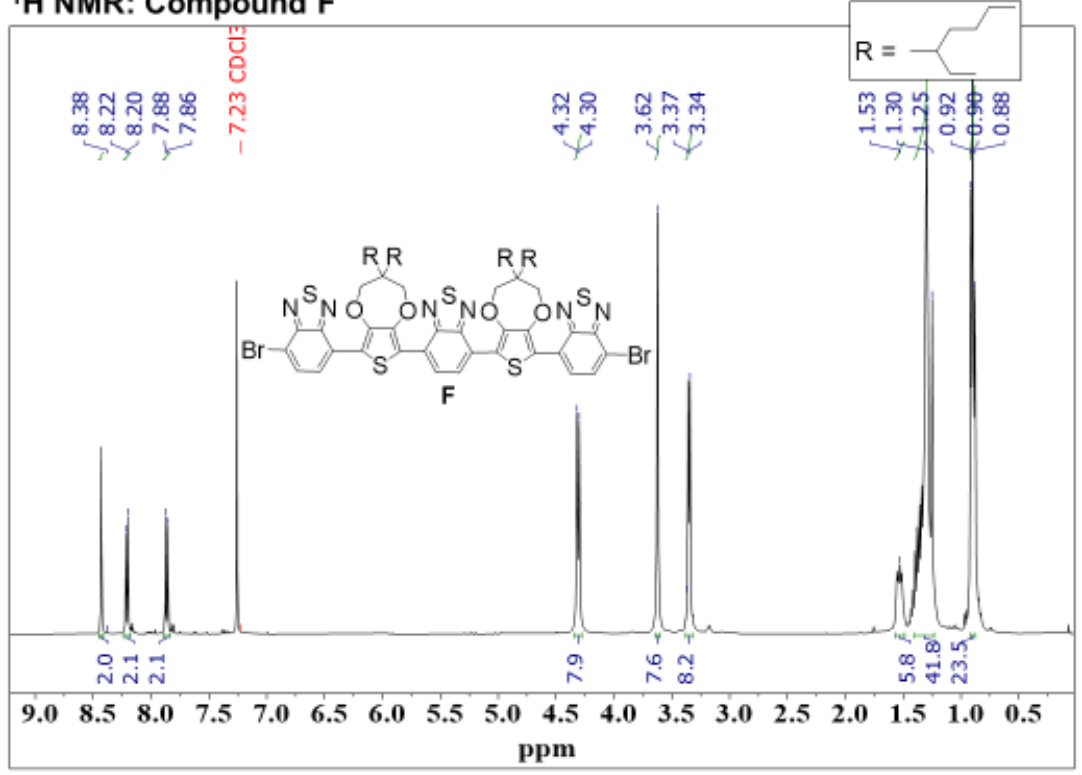

4,7-bis(8-(7-bromobenzo[c][1,2,5] thiadiazol-4-yl)-3,3-bis(((2-ethylhexyl)oxy)methyl)-3,4-dihydro-2H-thieno[3,4b][1,4]dioxepin-6-yl)benzo[c][1,2,5] thiadiazole ( $\left.{ }^{2} \mathrm{H} \mathrm{NMR}, \mathrm{CDCl}_{3}, 400 \mathrm{MHz}\right)$

${ }^{13} \mathrm{C}$ NMR: Compound F

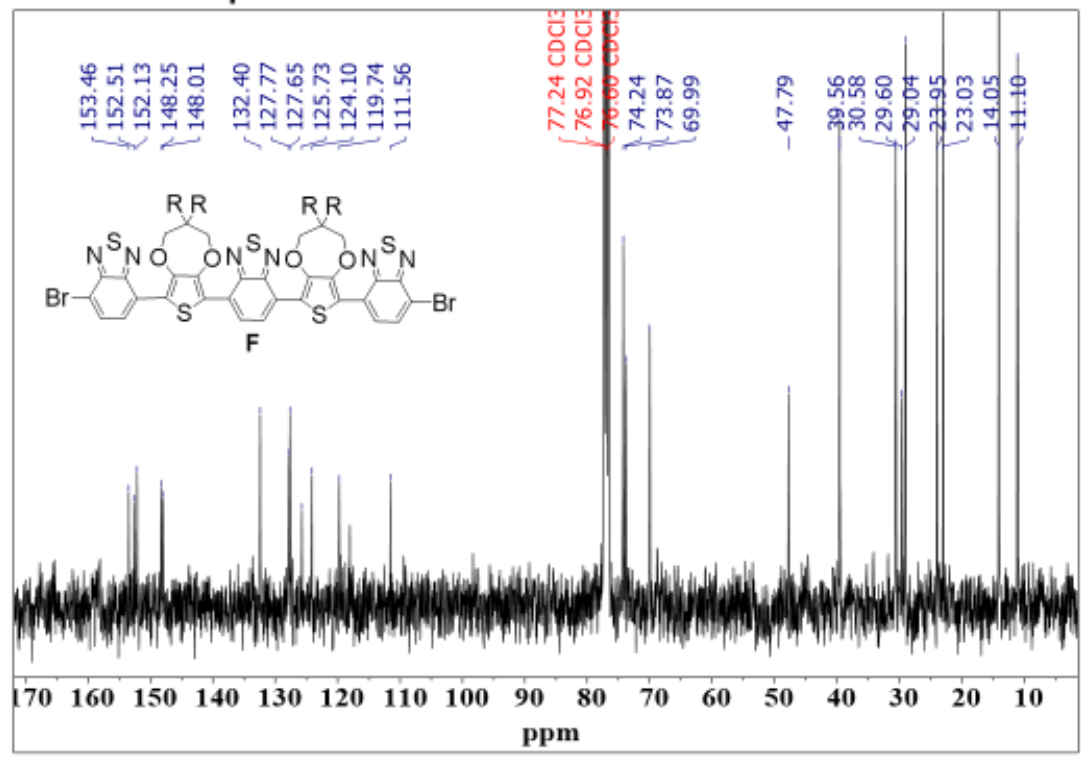

4,7-bis(8-(7-bromobenzo[c][1.2,5] thiadiazol-4-yl)-3,3-bis(((2-ethylbexyl)oxy)methyl)-3,4-dilhydro-2H-thieno[3,4b][1,4]dioxepin-6-yl)benzo[c][1,2,5] thiadiazole $\left({ }^{13} \mathrm{CNMR}, \mathrm{CDCl}_{3}, 101 \mathrm{MHz}\right)$ 
${ }^{1}$ H NMR: Compound G

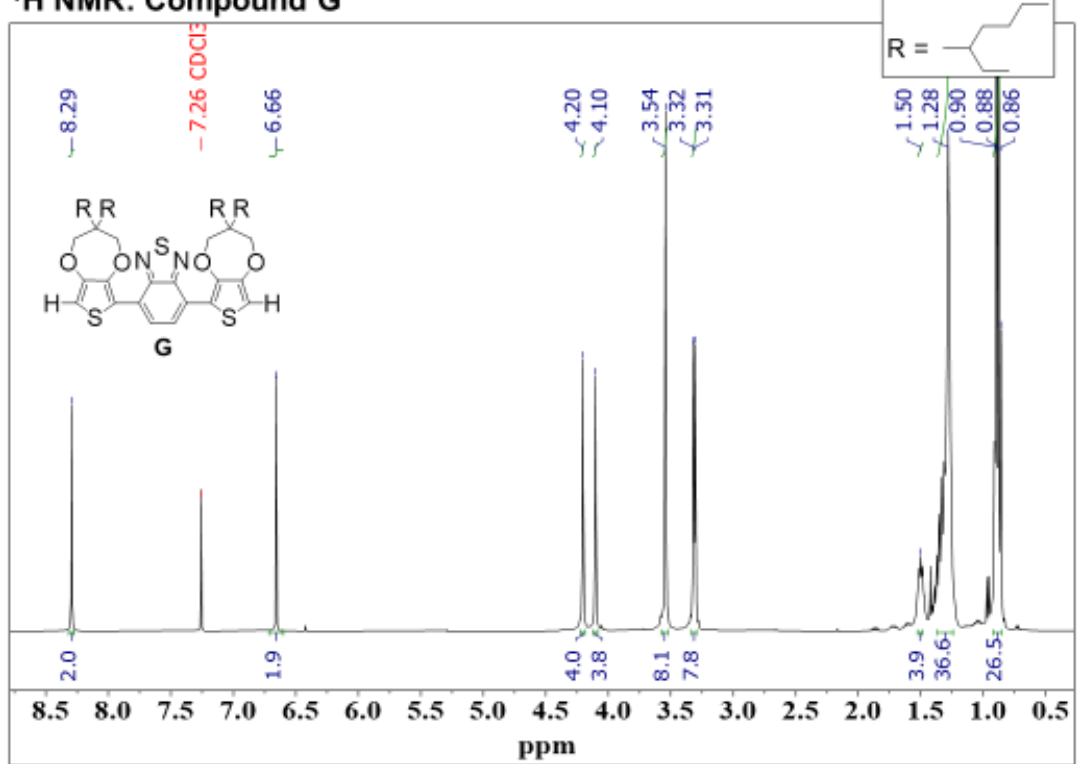

4,7-bis(3,3-bis(((2-ethylhexyl)oxy)methyl)-3,4-dilhydro-2H-thieno[3,4-b][1,4]dioxepin-6-yl)benzo[c][1,2,5] thiadiazole ( ${ }^{\mathrm{H}} \mathrm{H}$ $\left.\mathrm{NMR}, \mathrm{CDCl}_{3}, 400 \mathrm{MHz}\right)$

${ }^{13} \mathrm{C}$ NMR: Compound G

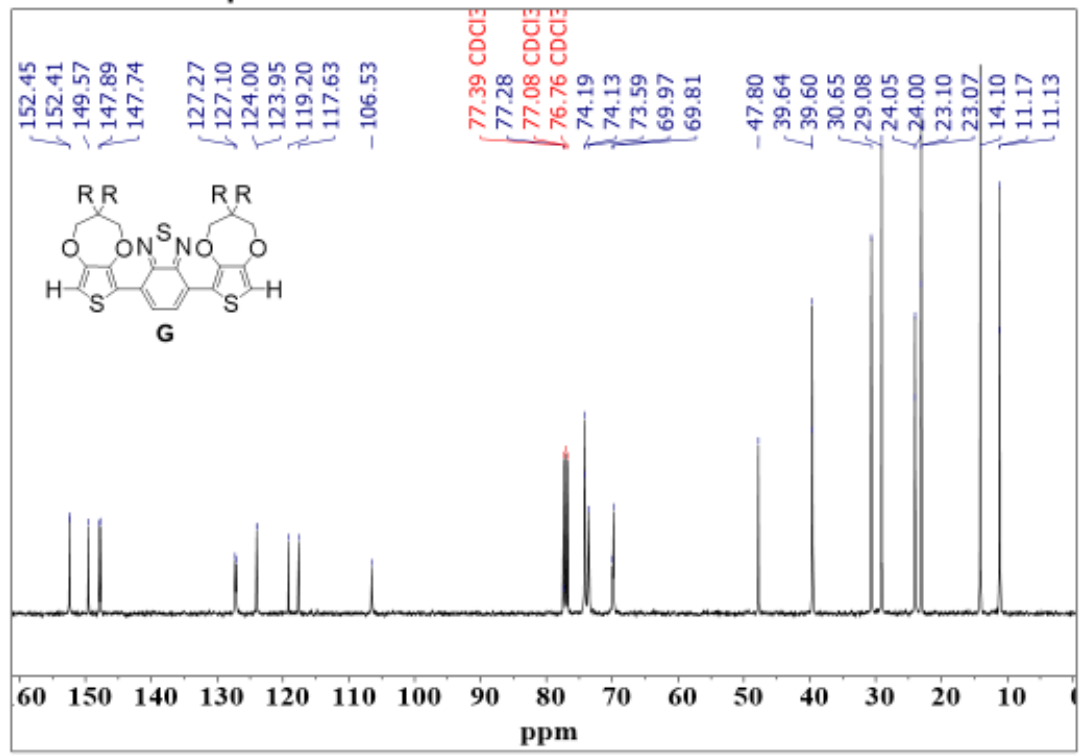

4,7-bis(3,3-bis(((2-ethylhexyl)oxy)methyl)-3,4-dilhydro-2H-thieno[3,4-b][1,4]dioxepin-6-yl)benzo[c] $[1,2,5]$ thiadiazole $\left({ }^{13} \mathrm{C}\right.$ $\left.\mathrm{NMR}, \mathrm{CDCl}_{3}, 101 \mathrm{MHz}\right)$ 
${ }^{1} \mathrm{H}$ NMR: Compound H

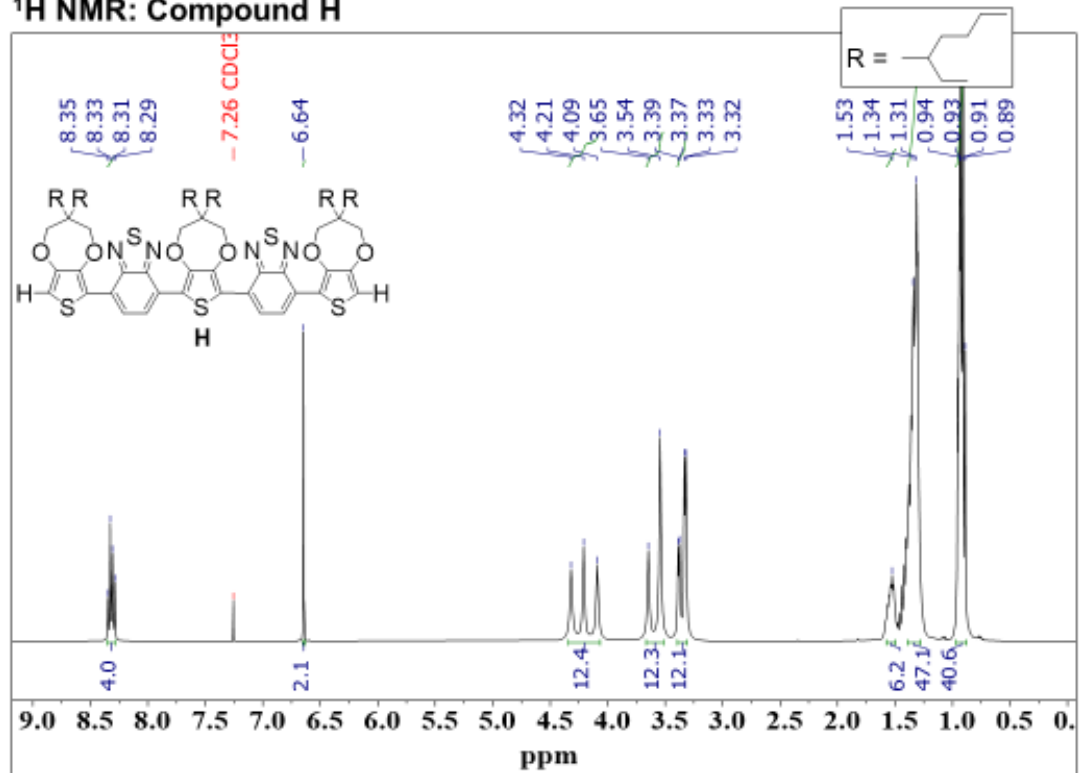

7,7'-(3,3-bis[([2-ethylhexyl)oxy]methyl)-3,4-dihydro-2H-thieno[3,4-b][1,4]dioxepine-6,8-diyl)bis[4-[3,3-bis(((2ethylhexyl)oxy]methyl)-3,4-dihydro-2H-thieno[3,4-b][1,4]dioxepin-6-yl) benzo[c][1,2,5]thiadiazole) ( ${ }^{3} \mathrm{H} \mathrm{NMR,} \mathrm{CDCl}_{3}$. $400 \mathrm{MHz})$

${ }^{13} \mathrm{C}$ NMR: Compound H

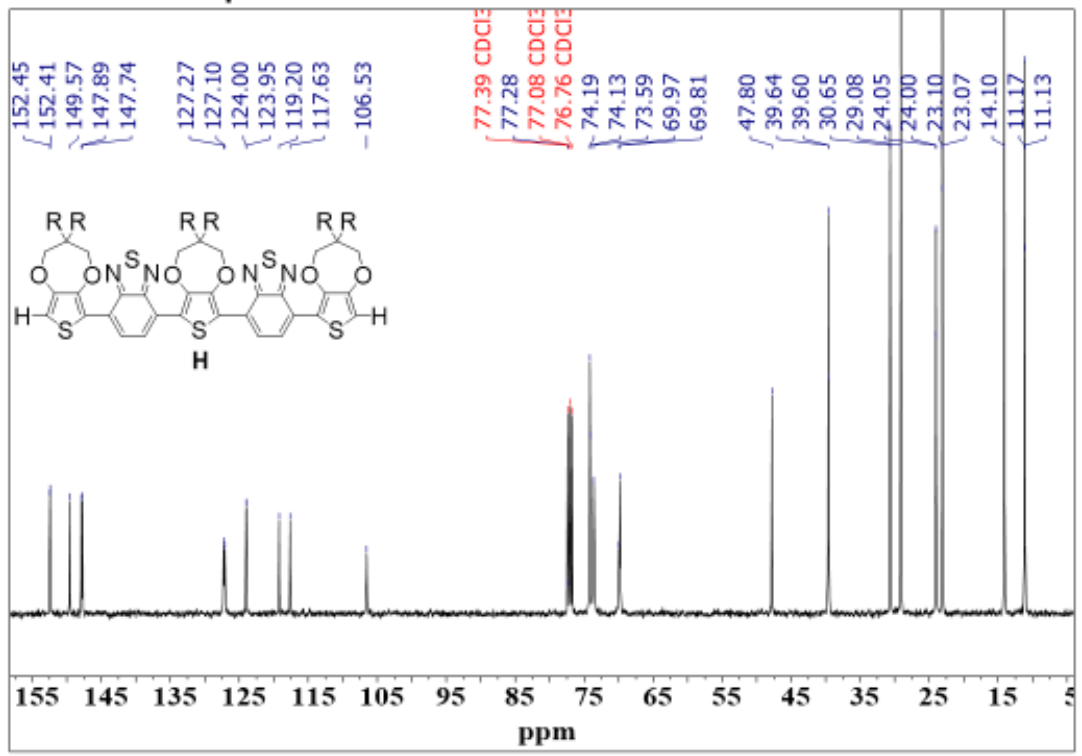

7,7'-(3,3-bis[([2-ethylhexyl)oxy)methyl)-3,4-dihydro-2H-thieno[3,4-b][1,4] dioxepine-6,8-diyl)bis [4-[3,3-bis(((2ethylhexyl|)oxy]methyl]-3,4-dihydro-2H-thieno[3,4-b][1,4]dioxepin-6-yl]benzo[c][1,2,5]thiadiazole) $\left({ }^{13} \mathrm{CNMR}, \mathrm{CDCl}_{3}\right.$ $101 \mathrm{MHz}$ ) 
${ }^{1}$ H NMR: Compound I

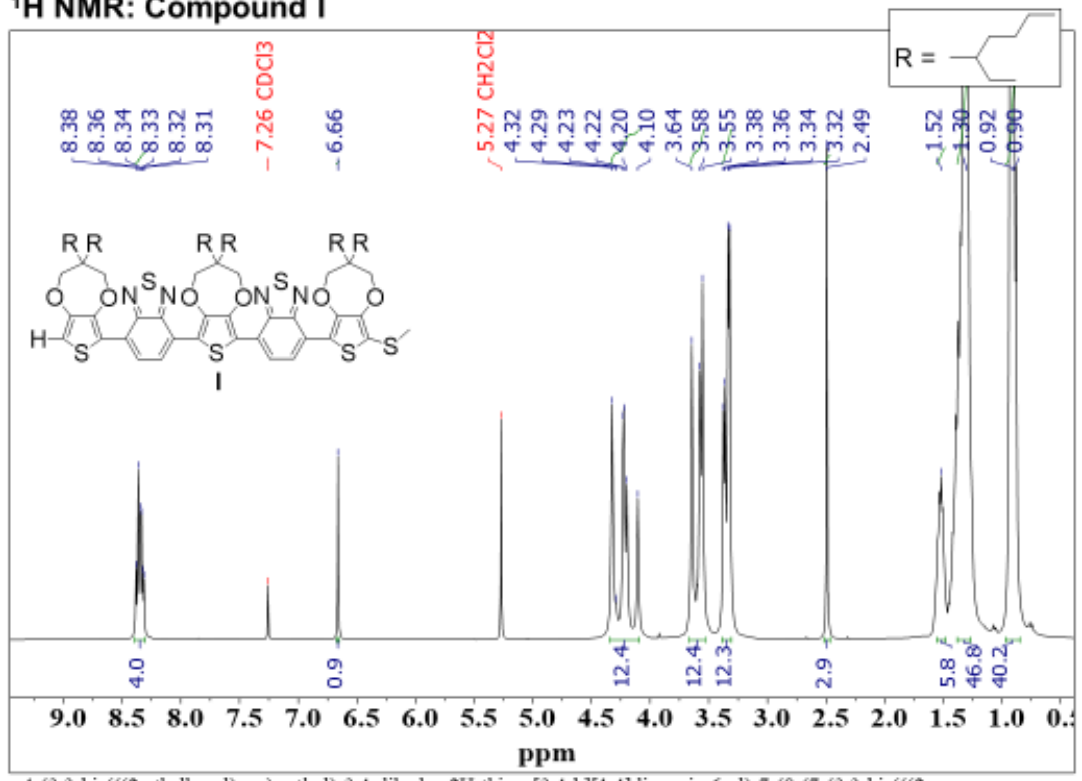

4-(3,3-bis(((2-ethylhexyl)oxy)methyl)-3,4-dihydro-2H-thieno[3,4-b][1,4]dioxepin-6-yl)-7-(8-(7-(3,3-bis(((2-

ethylhexyl)oxy)methyl)-8-(methylthio)-3,4-dilyydro-2H-thieno $[3,4-b][1,4]$ dioxepin-6-yl)benzo[c][1,2,5] thiadiazol-4-yl)3,3-bis((2-ethylhexyl)oxy)methyl)-3,4-dilydro-2H-thieno[3,4-b][1,4]dioxepin-6-yl)benzo[c] [1,2,5] thiadiazole ( ${ }^{\mathrm{H}} \mathrm{H}$ NMR. $\left.\mathrm{CDCl}_{3}, 400 \mathrm{MHz}\right)$

\section{${ }^{13}$ C NMR: Compound I}

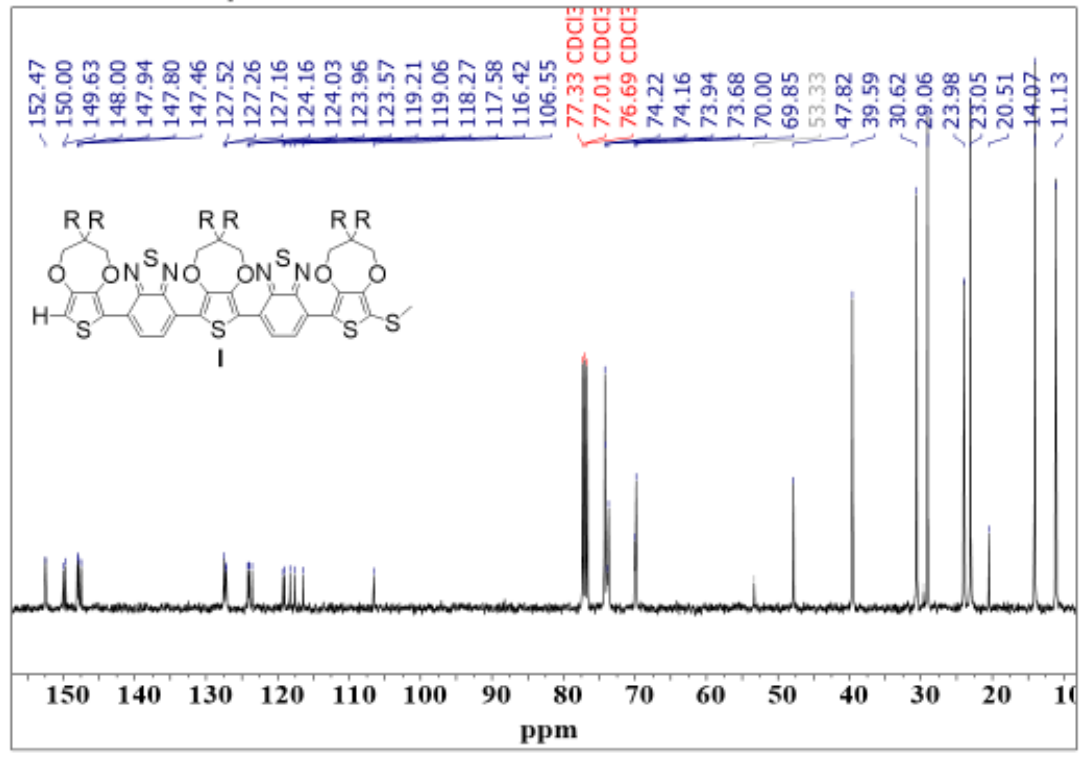

4-(3,3-bis(((2-ethylhexyl)oxy)methyl)-3,4-dihydro-2H-thieno[3,4-b][1,4]dioxepin-6-yl)-7-(8-(7-(3,3-bis(((2ethylhexyl)oxy)methyl)-8-(methylthio)-3,4-dilhydro-2H-thieno[3,4-b][1,4] dioxepin-6-yl)benzo[c] [1,2,5] thiadiazol-4-yl)3,3-bis(((2-ethylhexyl)oxy)methyl)-3,4-dilydro-2H-thieno[3,4-b][1,4]dioxepin-6-yl)benzo[c] $[1,2,5]$ thiadiazole $\left({ }^{15} \mathrm{C} N M R\right.$, $\left.\mathrm{CDCl}_{3}, 101 \mathrm{MHz}\right)$ 
${ }^{1} \mathrm{H}$ NMR: Compound J

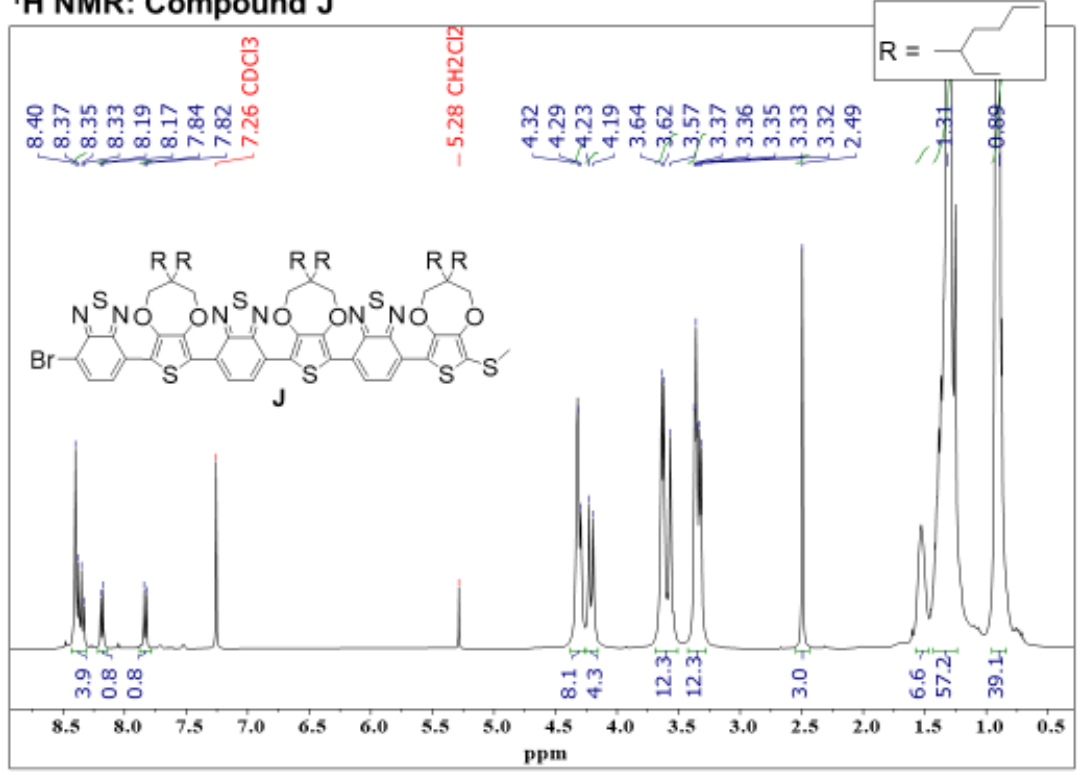

7,7'-(3,3-bis(((2-ethylhexyl)oxy)methyl)-3,4-dilhydro-2H-thieno[3,4-b][1,4]dioxepine-6,8-diyl)bis(4-(3,3-bis(((2ethylhexyl)oxy)methyl)-3,4-dilhydro-2H-thieno[3,4-b][1,4]dioxepin-6-yl)benzo[c][1,2,5] thiadiazole) ('H NMR, $\mathrm{CDCl}_{3}$ $400 \mathrm{MHz})$

${ }^{13} \mathrm{C}$ NMR: Compound J

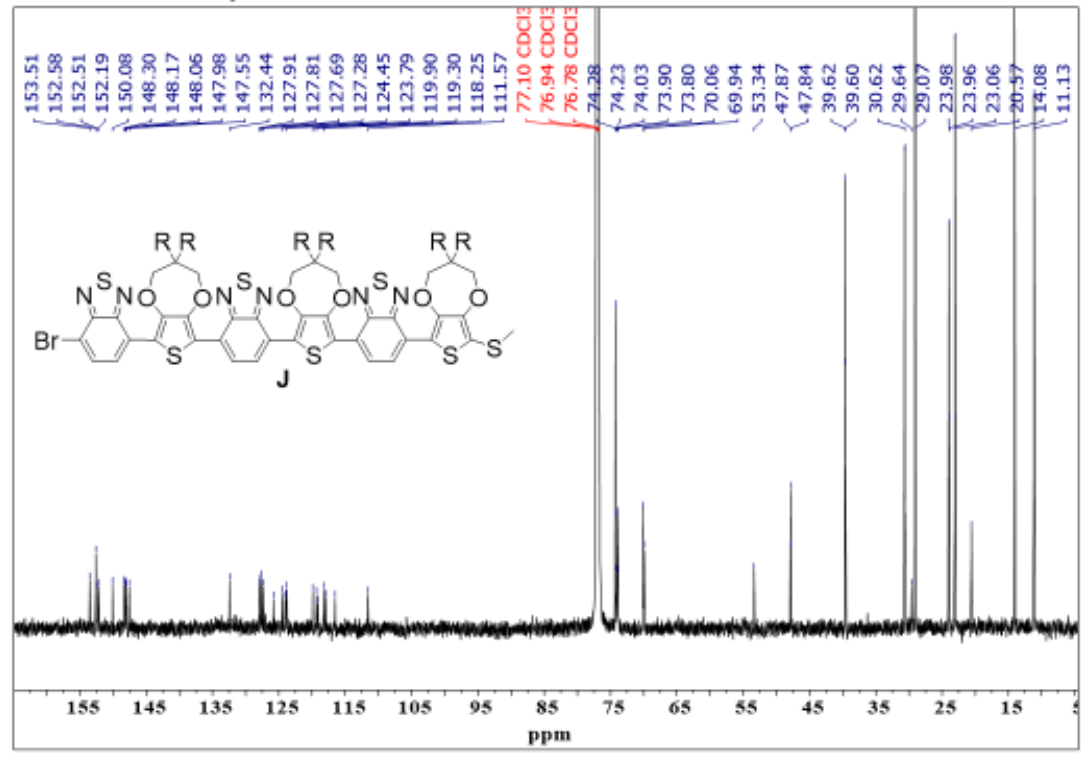

7,7'-(3,3-bis(((2-ethylhexyl)oxy)methyl)-3,4-dilydro-2H-thieno[3,4-b][1,4]dioxepine-6,8-diyl)bis(4-(3,3-bis(((2ethylhexyl)oxy)methyl)-3,4-dilhydro-2H-thieno[3,4-b][1,4]dioxepin-6-yl)benzo[c][1,2,5] thiadiazole) $\left({ }^{13} \mathrm{C} \mathrm{NMR}, \mathrm{CDCl}\right.$. $101 \mathrm{MHz}$ ) 
${ }^{1} \mathrm{H}$ NMR: Compound K

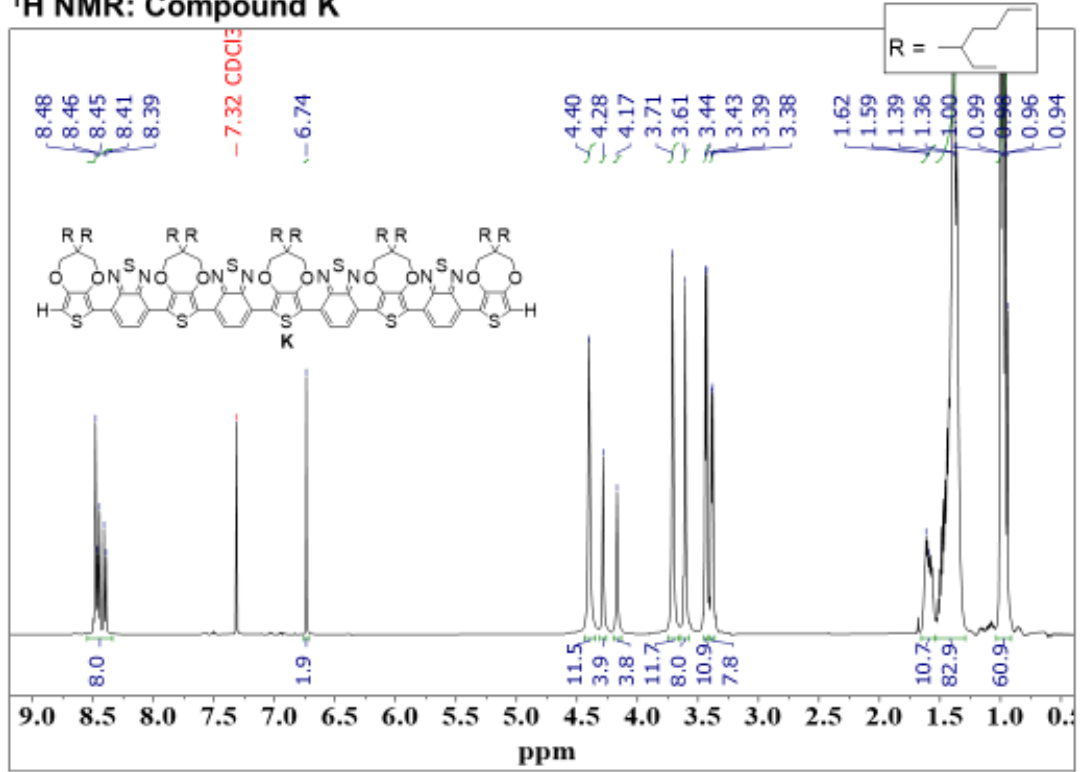

7,7'-(3,3-bis(((2-ethylhexyl)oxy)methyl)-3,4-dilydro-2H-thieno[3,4-b][1,4]dioxepine-6,8-diyl)bis(4-(8-(7)-(3,3-bis(((2ethylhexyl)oxy)methyl)-3,4-dilhydro-2H-thieno[3,4-b][1,4]dioxepin-6-yl)benzo[c][1,2,5] thiadiazol-4-yl)-3,3-bis(((2ethylhexyl)oxy)methyl)-3,4-dihydro-2H-thieno[3,4-b][1,4]dioxepin-6-yl)benzo[c][1,2,5]thiadiazole) ( ${ }^{2} \mathrm{H} \mathrm{NMR}, \mathrm{CDCl}_{3 .}$. $500 \mathrm{MHz})$

${ }^{13} \mathrm{C}$ NMR: Compound $\mathrm{K}$

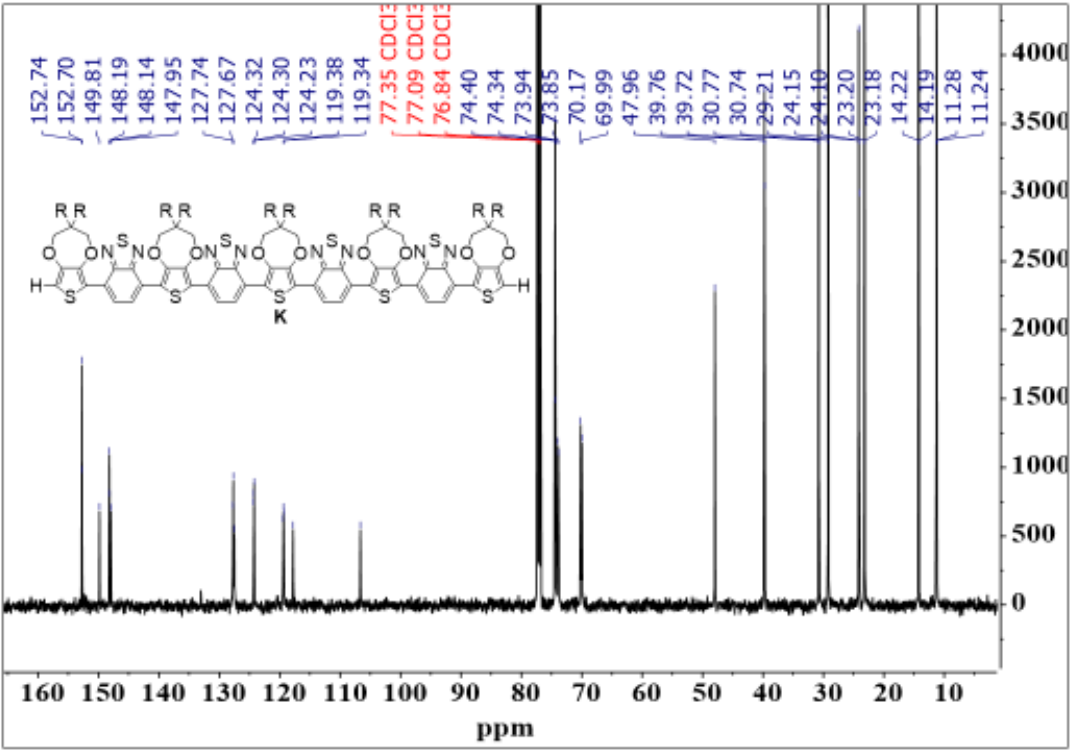

7,7'-(3,3-bis(((2-ethylhexyl)oxy)methyl)-3,4-dilydro-2H-thieno[3,4-b][1,4]dioxepine-6,8-diyl)bis(4-(8-(7-(3,3-bis(((2ethylhexyl)oxy)methyl)-3.4-dilhydro-2H-thieno[3,4-b][1.4] dioxepin-6-yl)benzo[c][1.2,5] thiadiazol-4-yl)-3,3-bis(((2ethylhexyl)oxy)methyl)-3,4-dihydro-2H-thieno[3,4-b][1,4]dioxepin-6-yl)benzo[c] $[1,2,5]$ thiadiazole) $\left({ }^{13} \mathrm{C} \mathrm{NMR,} \mathrm{CDCl}_{3}\right.$, $126 \mathrm{MHz}$ ) 
${ }^{1} \mathrm{H}$ NMR: $\mathbf{n = 3}$

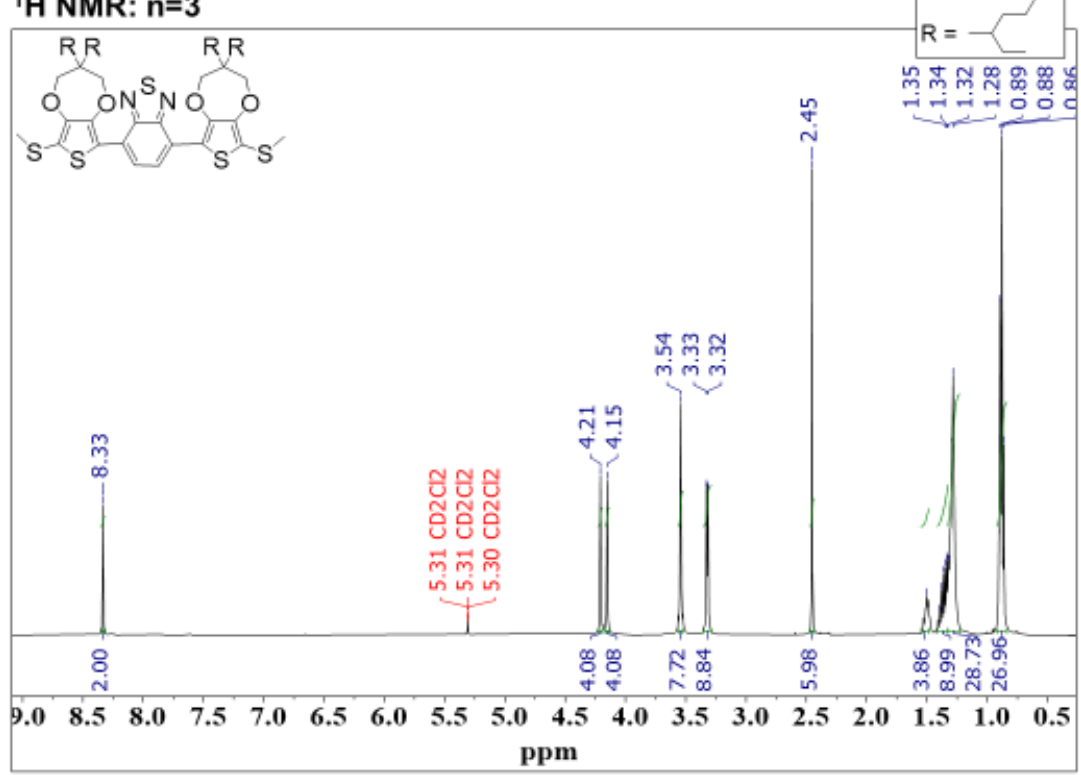

4,7-bis(3,3-bis(((2-ethylhexyl)oxy)methyl)-8-(methylthio)-3,4-dihydro-2H-thieno[3,4-b][1,4]dioxepin-6yl)benzo[c] $[1,2,5]$ thiadiazole ( $\left.{ }^{\mathrm{H}} \mathrm{NMR}, \mathrm{CD}_{2} \mathrm{Cl}_{2}, 500 \mathrm{MHz}\right)$

${ }^{13}$ C NMR: $n=3$

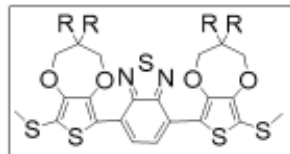

ศุตู่

\%ัฒ

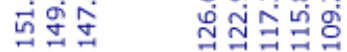

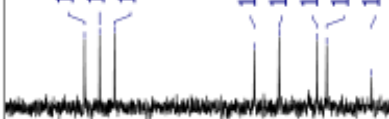$$
\begin{array}{|llllllllllllllllll}
\hline 160 & 150 & 140 & 130 & 120 & 110 & 100 & 90 & 80 & 70 & 60 & 50 & 40 & 30 & 20 & 10
\end{array}
$$
ppm

4,7-bis(3,3-bis(((2-ethylhexyl)oxy)methyl)-8-(methylthio)-3,4-dihydro-2H-thieno[3,4-b][1,4]dioxepin-6yl)benzo[c] $][1,2,5]$ thiadiazole ( ${ }^{5} \mathrm{C} \mathrm{NMR}, \mathrm{CD}_{2} \mathrm{Cl}_{2}, 126 \mathrm{MHz}$ ) 
${ }^{1} \mathrm{H}$ NMR: $\mathrm{n}=\mathbf{5}$

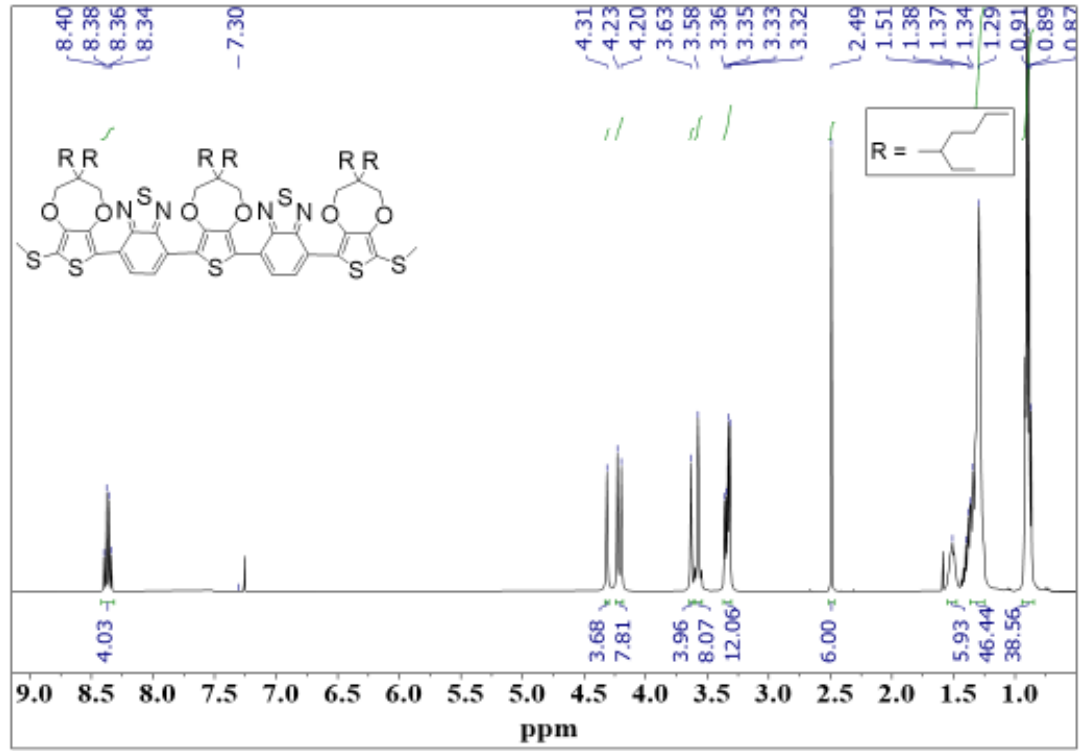

7,7'-(3,3-bis(((2-ethylhexyl)oxy)methyl)-3,4-dilhydro-2H-thieno[3,4-b][1,4]dioxepine-6,8-diyl)bis(4-(3,3-bis(((2ethylhexyl)oxy)methyl)-8-(methylthio)-3,4-dilhydro-2H-thieno[3,4-b][1,4]dioxepin-6-yl)benzo[c][1,2,5] thiadiazole)( ${ }^{2} \mathrm{H}$ $\left.\mathrm{NMR}, \mathrm{CDCl}_{3}, 400 \mathrm{MHz}\right)$

${ }^{13} \mathrm{C}$ NMR: $\mathrm{n}=\mathbf{5}$

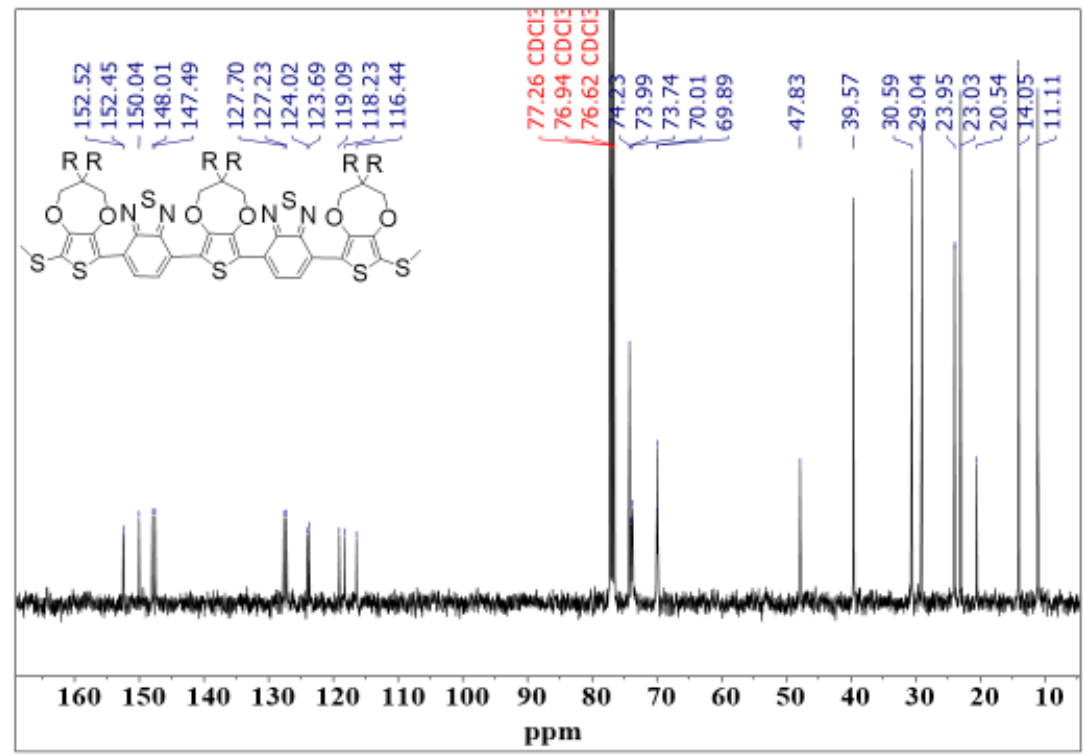

7,7'-(3,3-bis(((2-ethylhexyl)oxy)methyl)-3,4-dilydro-2H-thieno[3,4-b][1,4]dioxepine-6,8-diyl)bis(4-(3,3-bis(((2ethylhexyl)oxy)methyl)-8-(methylthio)-3,4-dilydro-2H-thieno[3,4-b][1,4]dioxepin-6-yl)benzo[c] $[1,2,5]$ thiadiazole) $\left({ }^{13} \mathrm{C}\right.$ $\mathrm{NMR}, \mathrm{CDCl}_{3}, 101 \mathrm{MHz}$ ) 


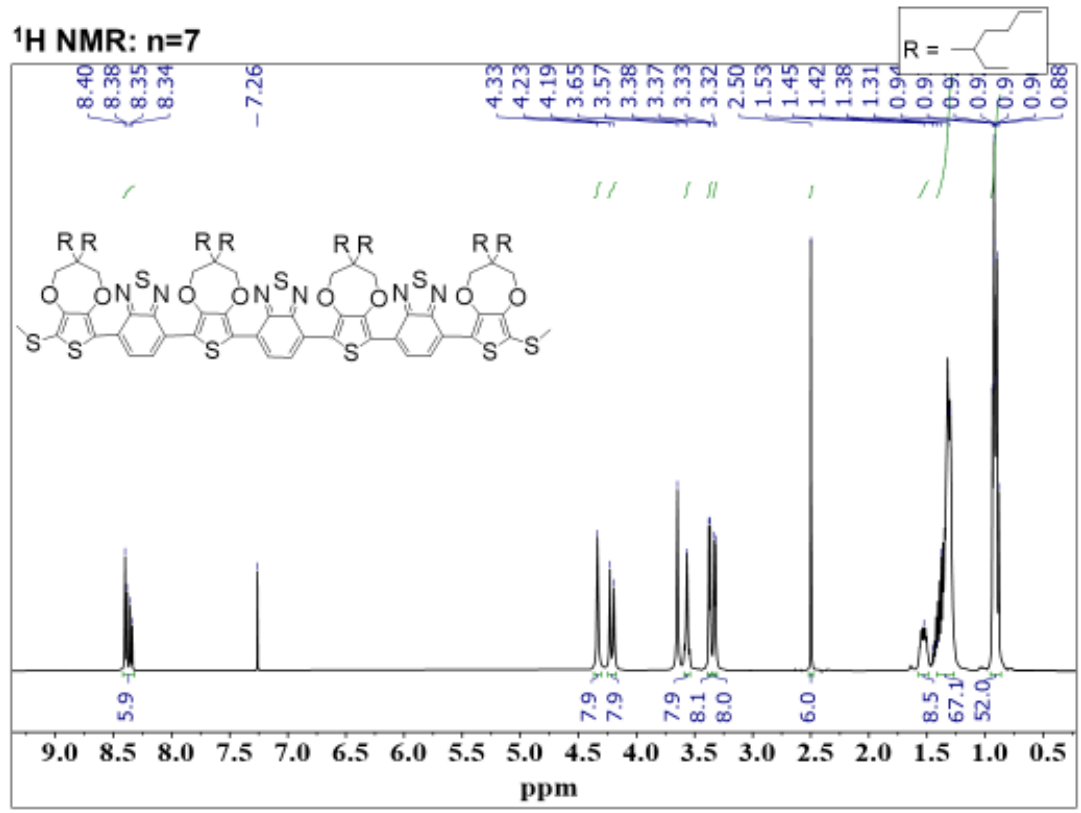

4,7-bis(8-(7-(3,3-bis((2-ethylhexyl)oxy)methyl)-8-(methylthio)-3,4-dihydro-2H-thieno[3,4-b][1,4]dioxepin-6yl)benzo[c] $[1,2,5]$ thiadiazol-4-yl)-3,3-bis $((2$-ethylhexyl $)$ oxy $)$ methyl $)-3,4-$ dihydro- $2 \mathrm{H}$-thieno $[3,4-\mathrm{b}][1,4]$ dioxepin- 6 yl)benzo[c] $[1,2,5]$ thiadiazole $\left({ }^{l} \mathrm{H} \mathrm{NMR}, \mathrm{CDCl}_{5}, 500 \mathrm{MHz}\right)$

${ }^{13} \mathrm{C}$ NMR: $\mathrm{n}=7$

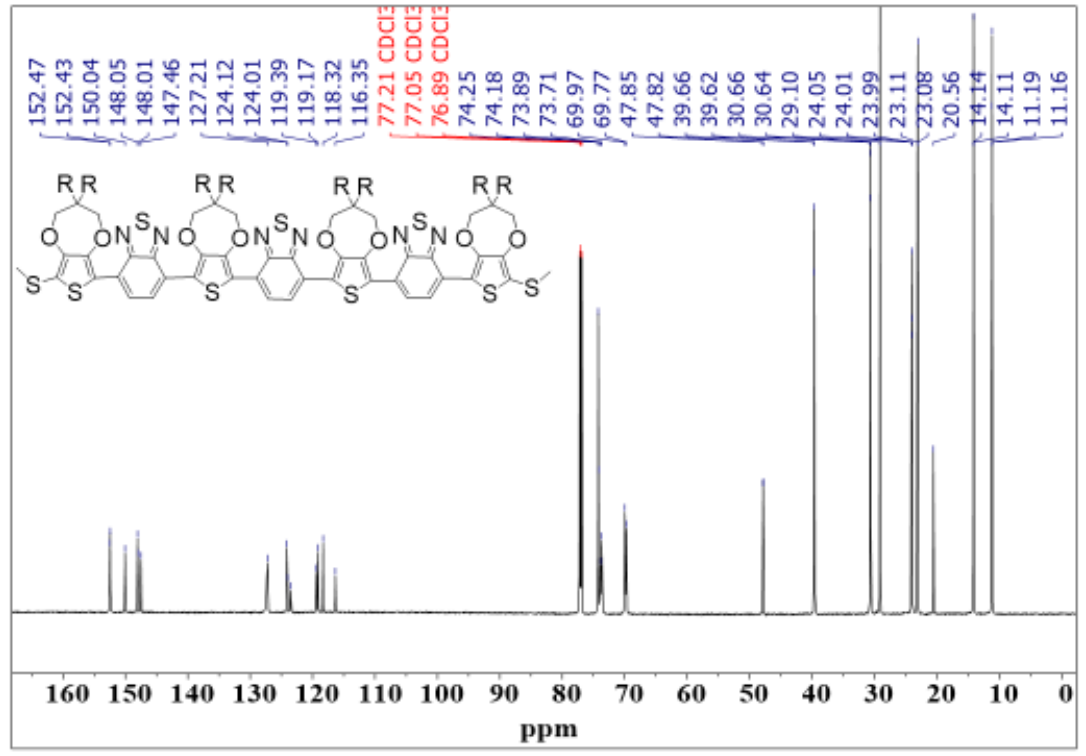

4,7-bis(8-(7-(3,3-bis((2-ethylhexyl)oxy)methyl)-8-(methylthio)-3,4-dihydro-2H-thieno[3,4-b] [1,4] dioxepin-6= yl)benzo[c] $[1,2,5]$ thiadiazol-4-yl)-3,3-bis $(((2-e t h y l h e x y l) o x y) m e t h y l)-3,4-d i h y d r o-2 \mathrm{H}-t h i e n o[3,4-b][1,4]$ dioxepin- 6 yl)benzo[c] $[1,2,5]$ thiadiazole (is $\left.\mathrm{C} \mathrm{NMR}, \mathrm{CDCl}_{3}, 201 \mathrm{MHz}\right)$ 
${ }^{1} \mathrm{H}$ NMR: $n=9$

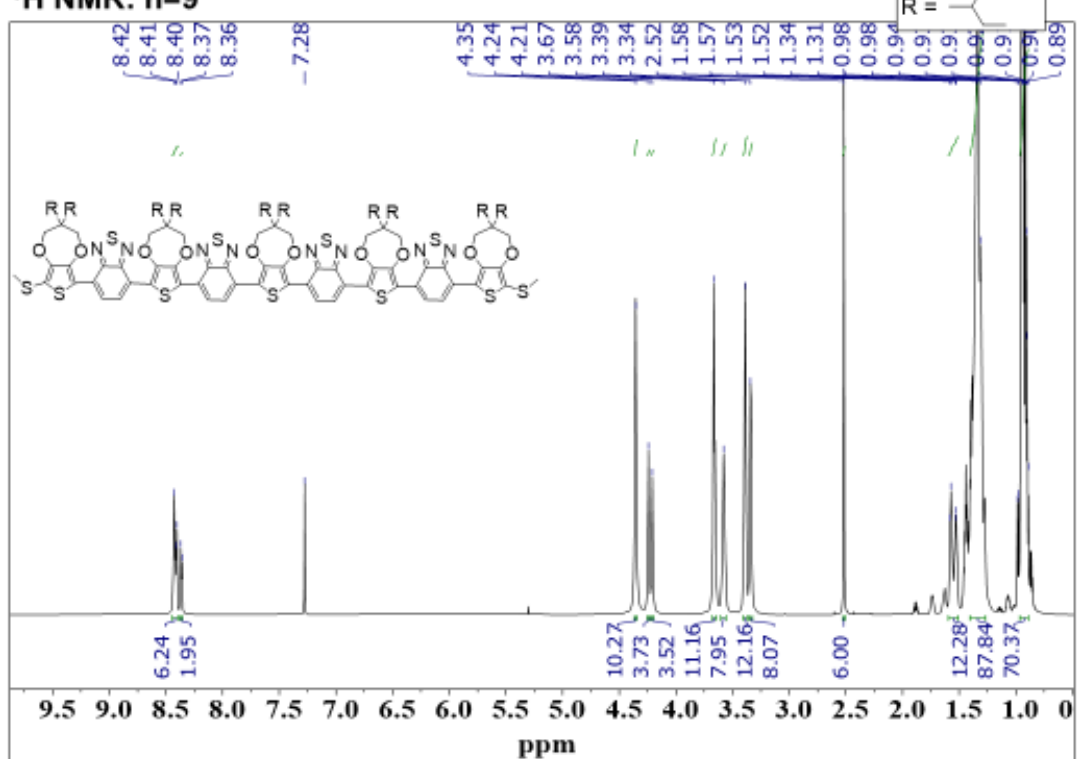

7,7'-(3,3-bis(((2-ethylhexyl)oxy)methyl)-3,4-dilhydro-2H-thieno[3,4-b][1,4]dioxepine-6,8-diyl)bis(4-(8-(7-(3,3-bis(((2ethylhexyl)oxy)methyl)-8-(methylthio)-3,4-dilyydro-2H-thieno[3,4-b][1,4]dioxepin-6-yl)benzo[c][1,2,5] thiadiazol-4-yl)3,3-bis(((2-ethylhexyl)oxy)methyl)-3,4-dilhydro-2H-thieno[3,4-b][1,4]dioxepin-6-yl)benzo[c][1,2,5] thiadiazole) ('H NMR, $\mathrm{CDCl}_{3}, 800 \mathrm{MHz}_{2}$

\section{${ }^{13}$ C NMR: $n=9$}

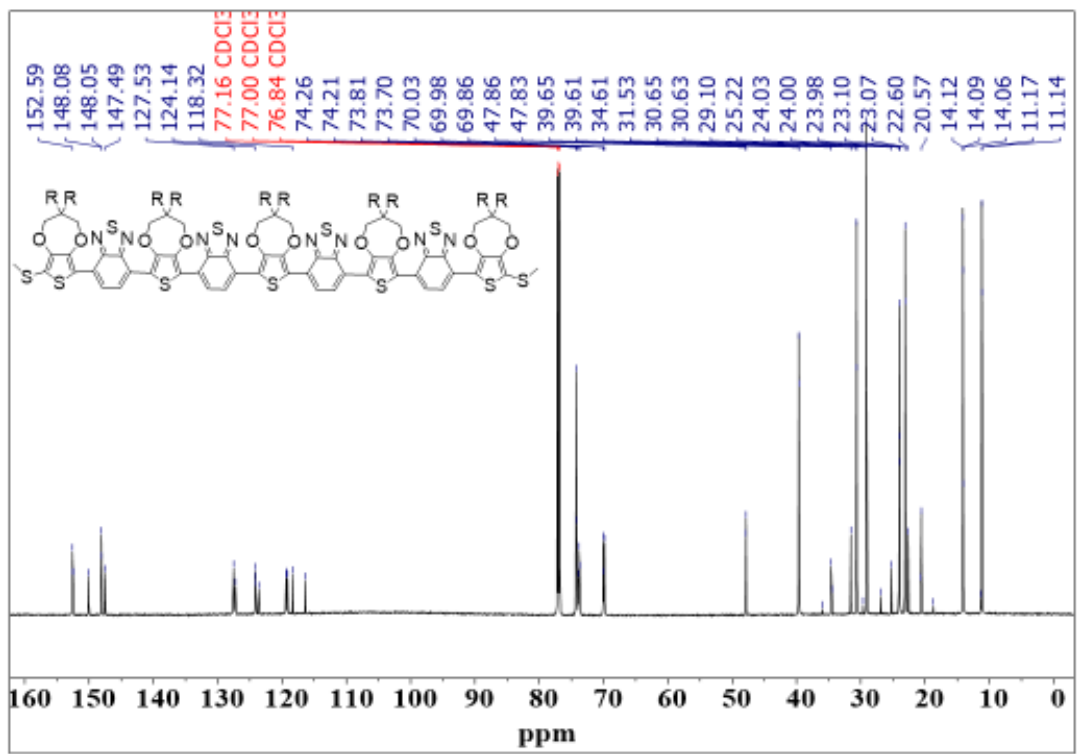

7,7'-(3,3-bis(((2-ethylhexyl)oxy)methyl)-3,4-dilydro-2H-thieno[3,4-b][1,4]dioxepine-6,8-diyl)bis(4-(8-(7-(3,3-bis(((2-) ethylhexyl)oxy)methyl)-8-(methylthio)-3,4-dilydro-2H-thieno[3,4-b][1,4]dioxepin-6-yl)benzo[c][1,2,5] thiadiazol-4-yl)3,3-bis(((2-ethylhexyl)oxy)methyl)-3,4-dihydro-2H-thieno[3,4-b][1,4]dioxepin-6-yl)benzo[c] $[1,2,5]$ thiadiazole) $\left({ }^{13} \mathrm{C}\right.$ NMR. $\left.\mathrm{CDCl}_{3}, 201 \mathrm{MHz}\right)$ 
${ }^{1} \mathrm{H}$ NMR: $n=15$

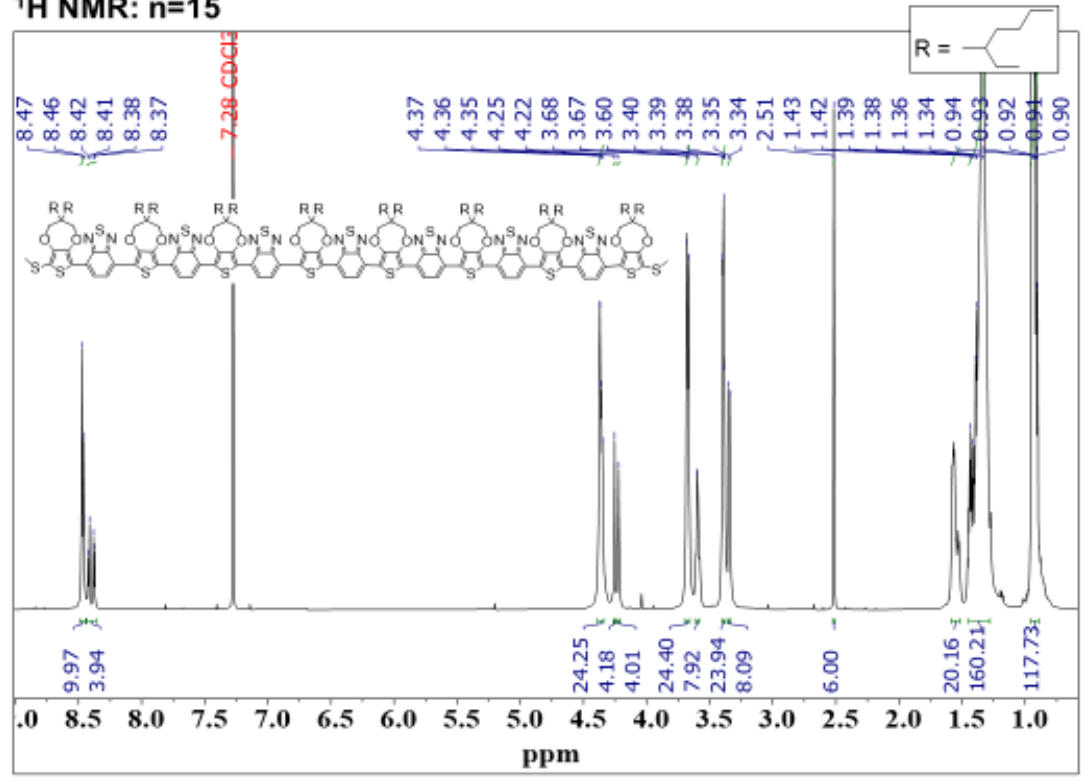

4,7-bis(8-(7-(8-(7--8-(7-(3,3-bis((2-ethylhexyl)oxy)methyl)-8-(methylthio)-3,4-dihydro-2H-thieno[3,4-b][1,4]dioxepin-6yl)benzo[c][1,2,5] thiadiazol-4-yl)-3,3-bis(((2-ethylhexyl)oxy)methyl)-3,4-dihydro- $2 \mathrm{H}$-thieno[3,4-b][1,4]dioxepin-6yl)benzo[c][1,2.5] thiadiazol-4-yl)-3,3-bis((2-ethylhexyl)oxy)methyl)-3,4-dihydro-2H-thieno[3,4-b][1,4]dioxepin-6yl)benzo[c][1,2,5] thiadiazol-4-yl)-3,3-bis(((2-ethylhexyl)oxy)methyl)-3,4-dihydro-2H-thieno[3,4-b][1,4]dioxepin-6yl)benzo[c][1,2,5]thiadiazole ( $\left.{ }^{i} \mathrm{H} \mathrm{NMR}, \mathrm{CDCl}_{3}, 800 \mathrm{MHz}\right)$

${ }^{13}$ C NMR: $n=15$

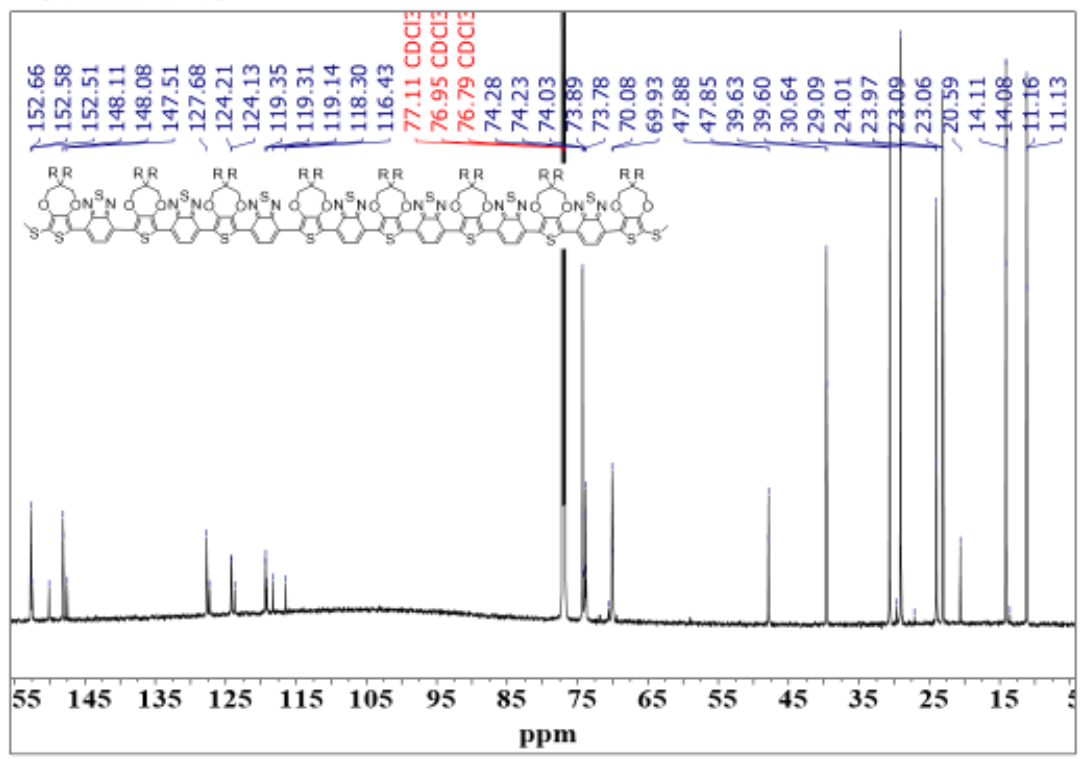

4,7-bis(8-(7-(8-(7)-(8-(7-(3,3-bis/((2-ethylhexyl)oxy)methyl)-8-(methylthio)-3,4-dihydro-2H-thieno[3,4-b][1,4]dioxepin-6yl)benzo[c] [1,2,5] thiadiazol-4-yl)-3,3-bis(((2-ethylhexyl)oxy)methyl)-3,4-dihydro-2H-thieno[3,4-b][1,4]dioxepin-6yl)benzo[c][1,2,5] thiadiazol-4-yl)-3,3-bis(((2-ethylhexyl)oxy)methyl)-3,4-dihydro-2H-thieno[3,4-b][1,4]dioxepin-6yl)benzo[c][1,2,5] thiadiazol-4-yl)-3,3-bis(((2-ethylhexyl)oxy)methyl)-3,4-dihydro-2H-thieno[3,4-b][1,4]dioxepin-6yl)benzo[c] $[1,2,5]$ thiadiazole $\left({ }^{15} \mathrm{C} \mathrm{NMR}, \mathrm{CDCl}_{3}, 201 \mathrm{MHz}\right)$ 
${ }^{1} \mathrm{H}$ NMR: $n=21$

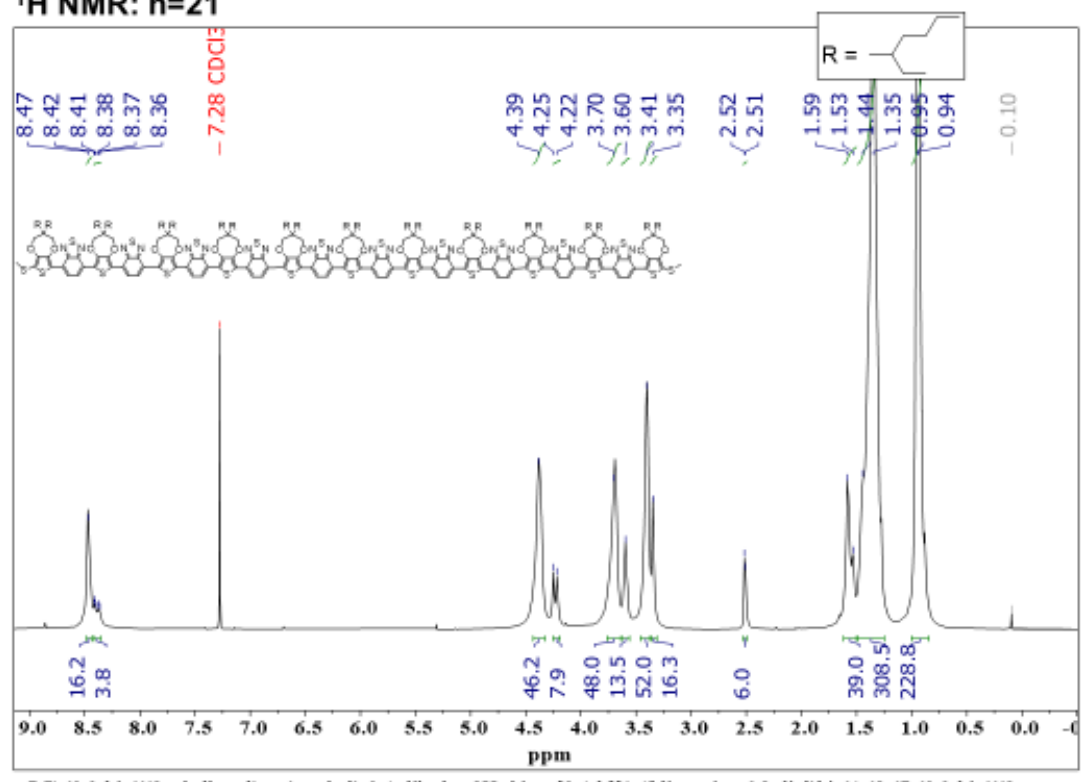

7,7'-(3,3-bis(((2-ethylhexyl)oxy)methyl)-3,4-dilyydro-2H-thieno[3,4-b][1,4]dioxepine-6,8-diyl)bis(4-(8-(7--(3,3-bis(((2ethylhexyl)oxy)methyl)-3.4-dihydro-2H-thieno[3,4-b][1.4] dioxepin-6-yl)benzo[c][1.2,5] thiadiazol-4-yl)-3,3-bis(((2ethylhexyl)oxy)methyl)-3,4-dihydro-2H-thieno[3,4-b][1,4]dioxepin-6-yl)benzo[c][1,2,5] thiadiazole) ('H NMR, $\mathrm{CDCl}_{5}$, $800 \mathrm{MHz}$ )

${ }^{13} \mathrm{C}$ NMR: $\mathrm{n}=\mathbf{2 1}$

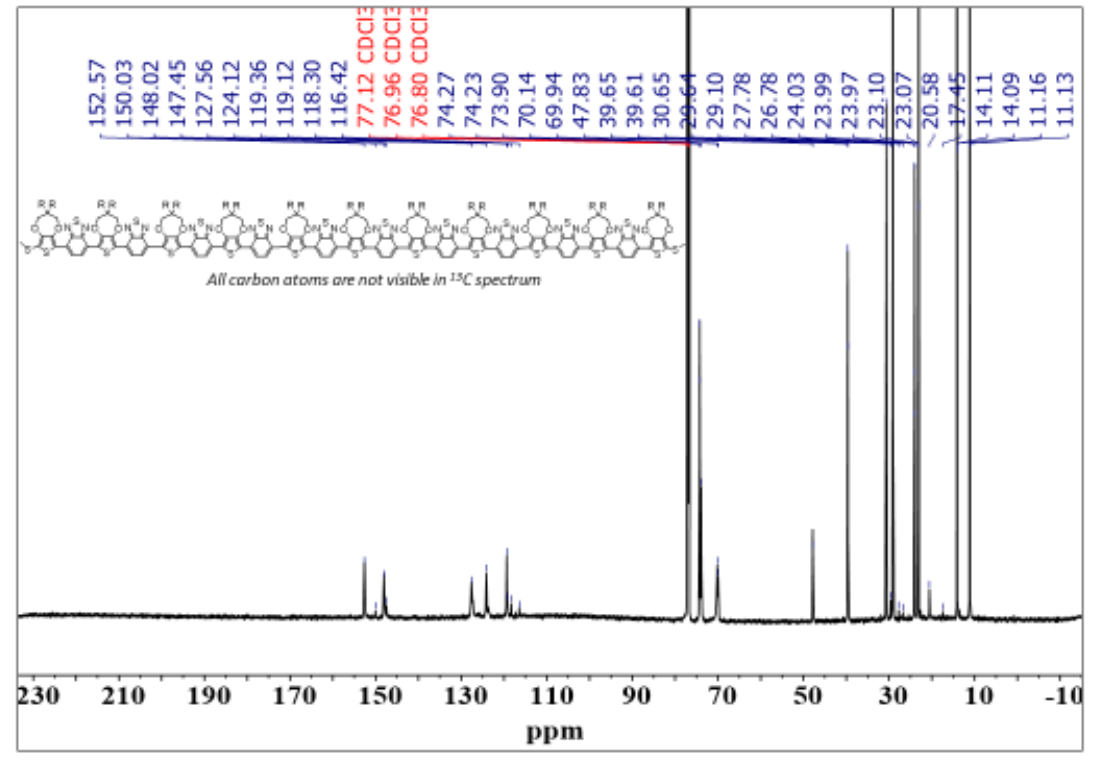

7,7'-(3,3-bis(((2-ethylhexyl)oxy)methyl)-3.4-dihydro-2H-thieno[3,4-b][1.4] dioxepine-6,8-diyl)bis(4-(8-(7)-(3,3-bis(((2ethylhexyl)oxy)methyl)-3,4-dihydro-2H-thieno[3,4-b][1,4]dioxepin-6-yl)benzo[c][1,2,5] thiadiazol-4-yl)-3,3-bis((2ethylhexyl)oxy)methyl)-3,4-dilhydro-2H-thieno[3,4-b][1,4]dioxepin-6-yl)benzo[c][1,2,5]thiadiazole) ( ${ }^{13} \mathrm{C} \mathrm{NMR}, \mathrm{CDCl}_{3}$, $201 \mathrm{MHz}$ ) 


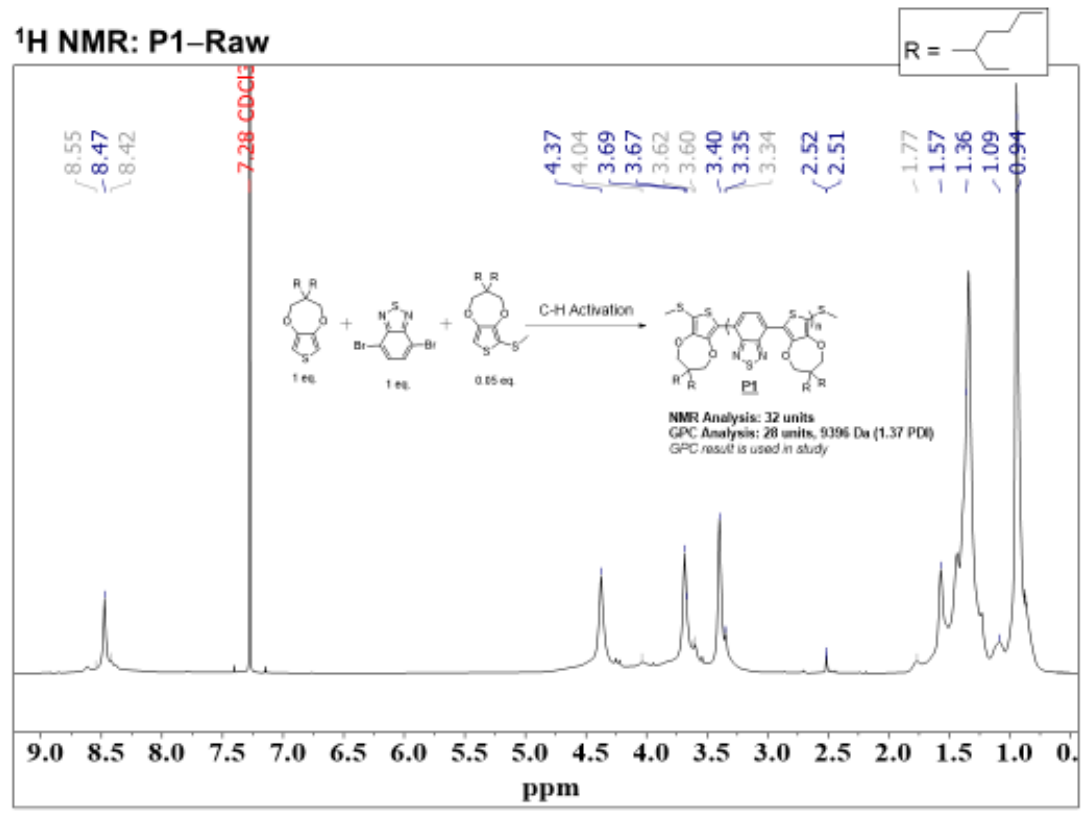

${ }^{1} \mathrm{H}$ NMR: P1-Peak Fitting

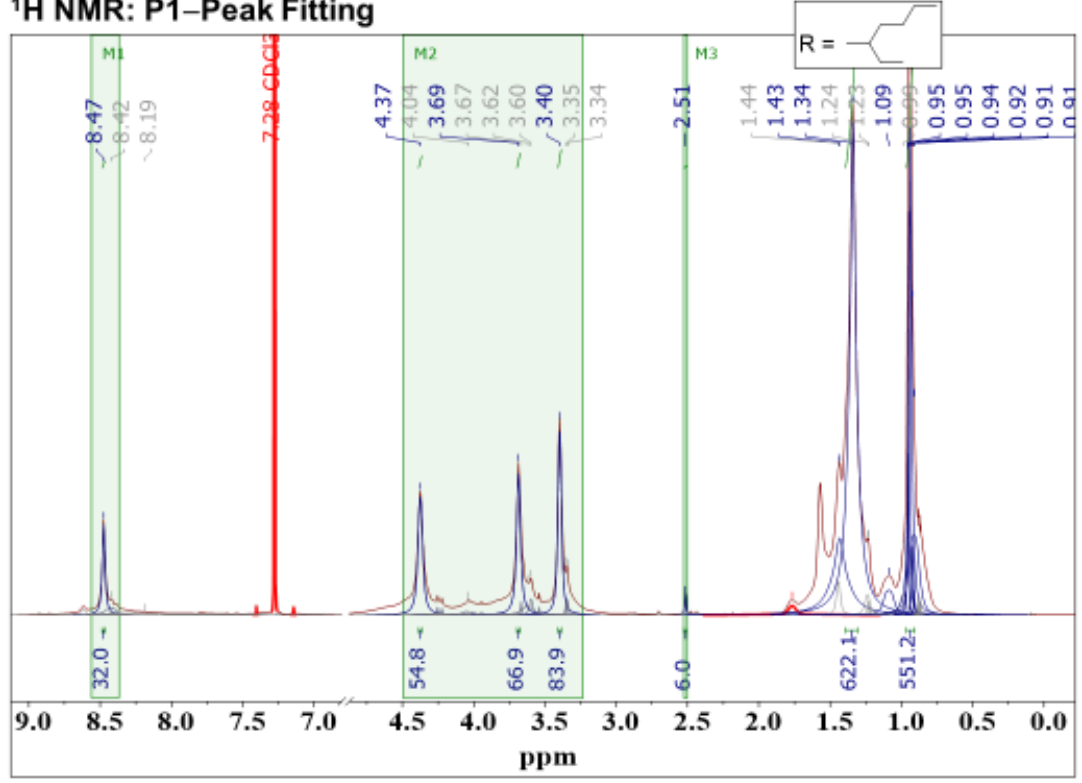




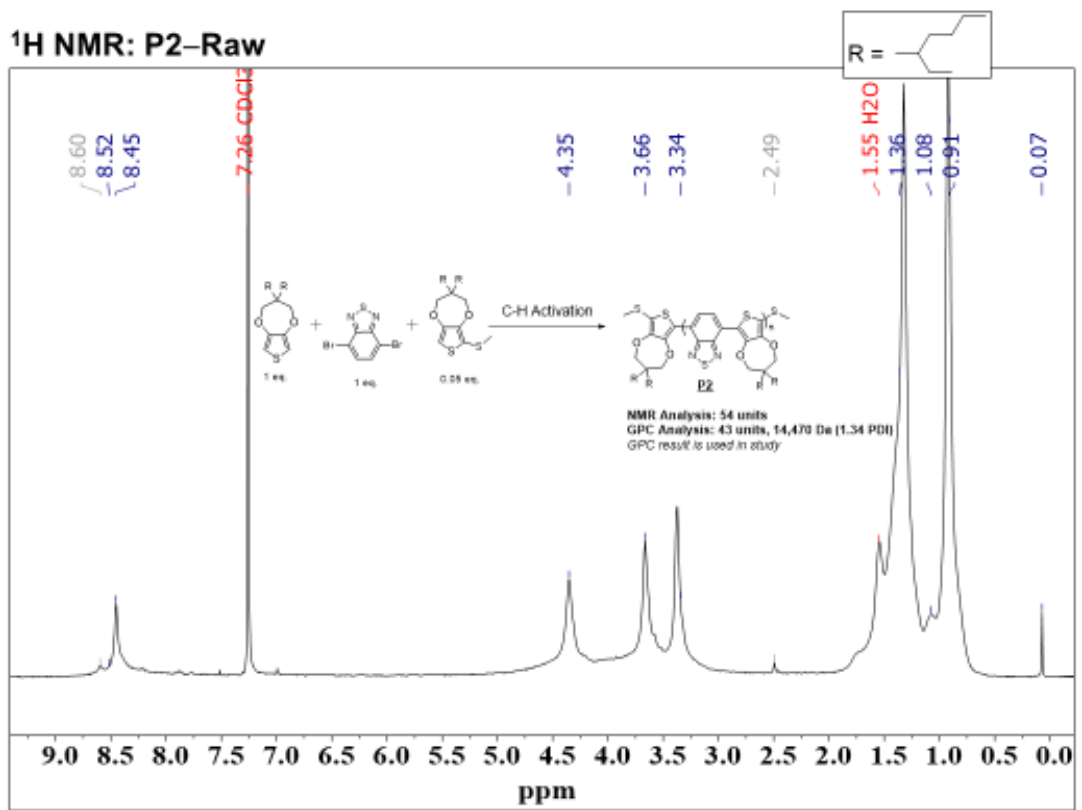

${ }^{1} \mathrm{H}$ NMR: P2-Peak Fitting

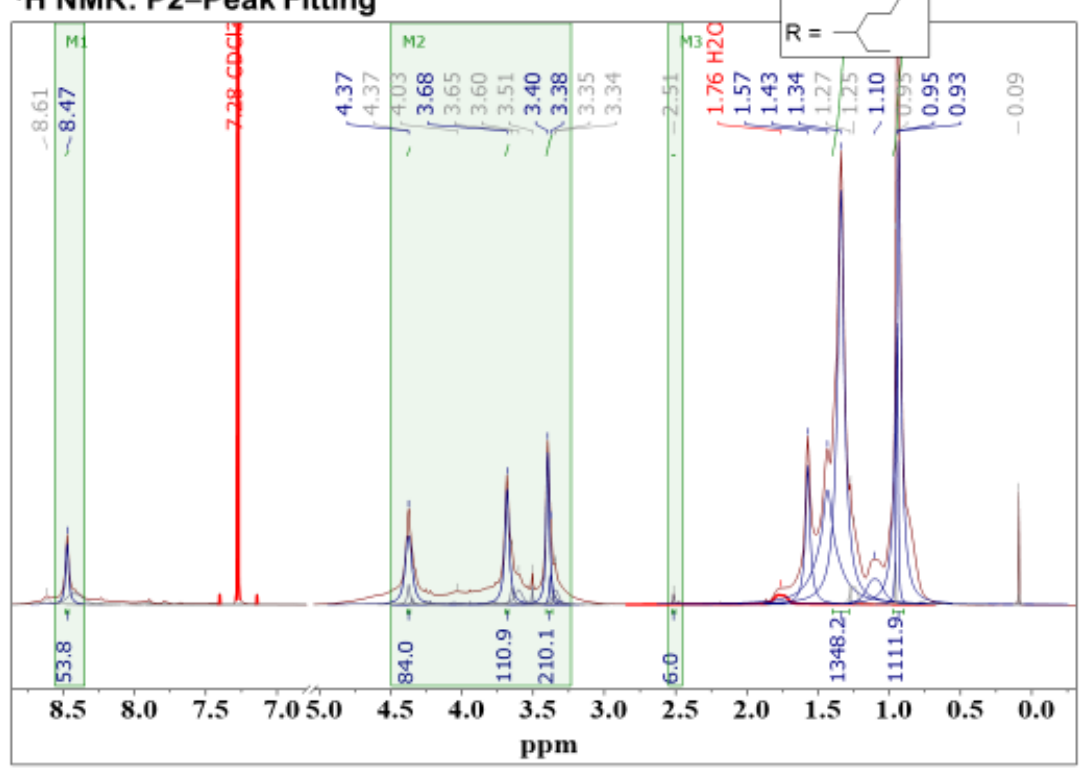




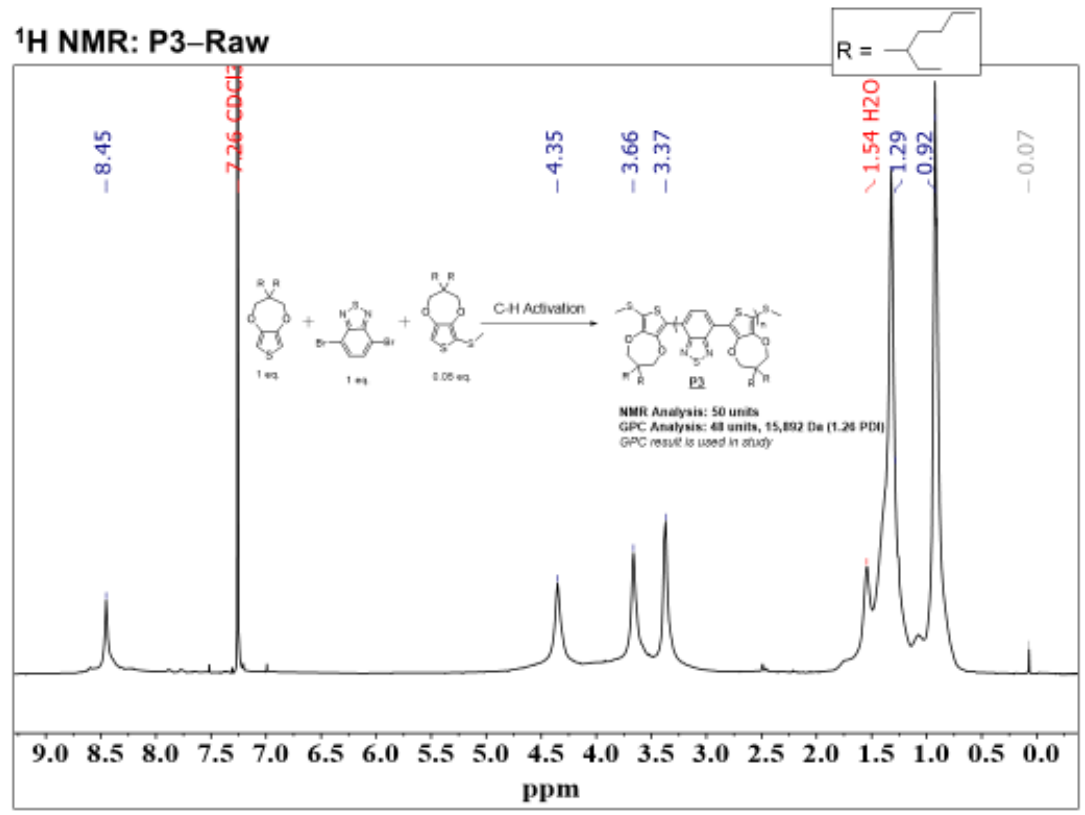

${ }^{1} \mathrm{H}$ NMR: P3-Peak Fitting

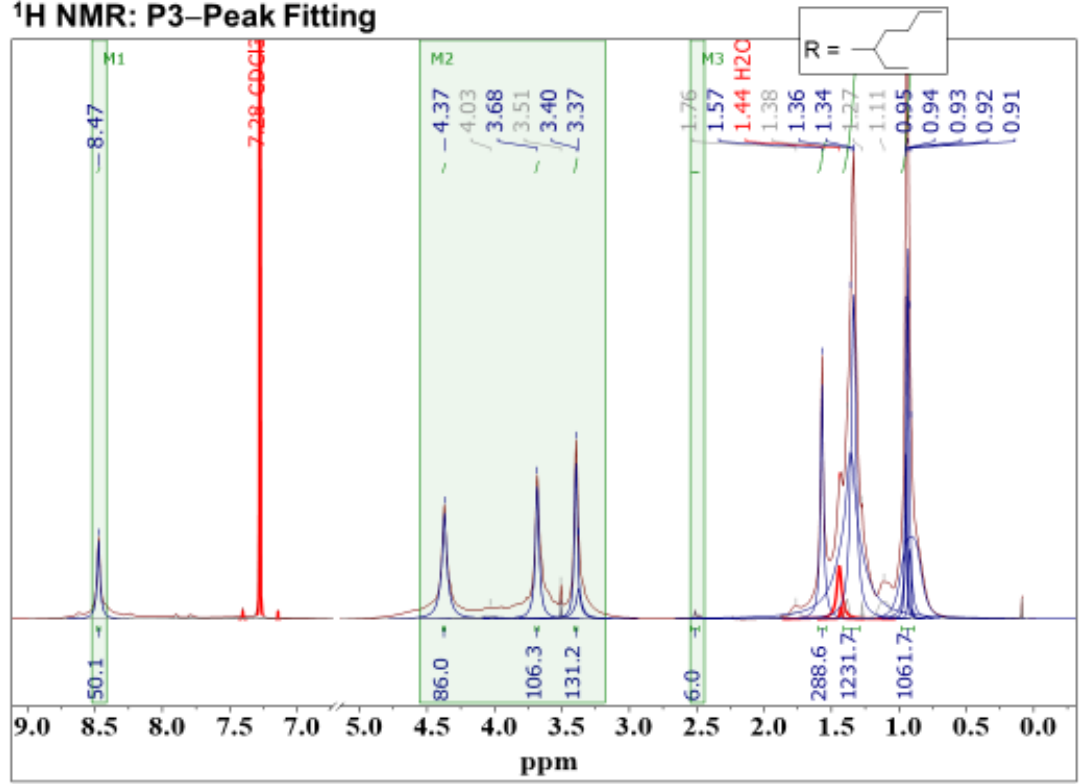

